\title{
Coronary Computed Tomography and Magnetic Resonance
}

\section{Imaging}

Birgit Kantor, MD, Eike Nagel, MD, Paul Schoenhagen, MD, Jörg Barkhausen, MD, and Thomas C. Gerber, MD

\section{Abstract}

Cardiac computed tomography and magnetic resonance are relatively new imaging modalities that can exceed the ability of established imaging modalities to detect present pathology or predict patient outcomes. Coronary calcium scoring may be useful in asymptomatic patients at intermediate risk. Computed tomographic coronary angiography is a first-line indication to evaluate congenitally abnormal coronary arteries and, along with stress magnetic resonance myocardial perfusion imaging, is useful in symptomatic patients with nondiagnostic conventional stress tests. Cardiac magnetic resonance is indicated for visualizing cardiac structure and function, and delayed enhancement magnetic resonance is a first-line indication for assessing myocardial viability. Imaging plaque and molecular mechanisms related to plaque rupture holds great promise for the presymptomatic detection of patients at risk for coronary events but is not yet suitable for routine clinical use.

\section{Vascular Biology of Coronary Artery Disease}

The high prevalence of myocardial infarction (MI) in previously asymptomatic patients is one of the major public health issues in the USA. ${ }^{1}$ In $50-65 \%$ of all patients, MI is the first clinical presentation of coronary artery disease (CAD). Most alarmingly, $38 \%$ of all first MIs are lethal. The dominant cause of MI is acute rupture of a vulnerable atherosclerotic plaque with subsequent complete occlusion of the coronary lumen by thrombus. ${ }^{2,3}$ Hence, there is tremendous need for imaging techniques or markers to noninvasively detect vulnerable plaques in asymptomatic patients who are at high risk for plaque rupture. Several morphologic and biomolecular key features of plaque vulnerability that can be identified by imaging are being evaluated for this purpose. ${ }^{4}$ A detailed understanding of plaque biology is essential to apply the most appropriate imaging technique to each clinical or research question.

\section{Morphologic and Biomolecular Features of Stable and Unstable Atherosclerotic Plaque}

The coronary artery wall is a highly specialized organ that consists of three distinct layers of cells with varying biological and physiological properties: the endothelium, the tunica media, and the tunica adventitia. The coronary artery wall has its own blood supply that is provided by vasa vasorum. 2,5

Pathogenesis of Coronary Atherosclerosis-Atherosclerosis follows a chronic progressive course from the initial subintimal fatty streak to diffuse stenotic disease. 6

The use of gadolinium chelates for cardiovascular magnetic resonance imaging as discussed in this article is off-label and not FDAapproved.

Eike Nagel is a consultant to Berlex/Schering and Bracco.

Paul Schoenhagen receives an honorarium from Takeda Pharmaceuticals (paid directly to charity and higher education).

Thomas C. Gerber is an associate editor of Mayo Clinic Proceedings.

The authors have no other conflicts of interest to disclose. 
Endothelial injury, resulting from toxic and oxidative damage from smoking and low-density lipoproteins or from hemodynamic shear forces, denudes the intima and activates endothelial cells. ${ }^{7}$ Vascular cell adhesion molecules (VCAM and selectins) facilitate adherence of inflammatory cells such as monocytes and lymphocytes to the endothelium and subsequent invasion of the media by these cells. ${ }^{1}$ Increased release of cytokines increases intimal permeability to these inflammatory cells ${ }^{8}$ (Fig 1). Phagocytosis of oxidized low-density lipoproteins distends the cytoplasm of local macrophages with numerous lipid vesicles, which on microscopy resemble coalesced bubbles or foam ("foam cells"). As the initial fatty streak expands, smooth muscle cells begin to migrate into the lesion and proliferate. These cells contribute to the production of proteolytic enzymes, matrix metalloproteinases, and collagenases, which cause breakdown of the elastic lamina between media and adventitia. In addition, lipids not bound to proteins precipitate as large needle-like crystals, causing a giant cell reaction within the plaque. ${ }^{7}$ In due course, the organized three-layer structure of the vessel wall disintegrates.

As the plaque thickens and the distance from the coronary artery lumen to the plaque core increases, the atheroma begins to outgrow its blood supply and becomes hypoxic. 9,10 Without sufficient oxygen supply, smooth muscle cells and other cells in the plaque perish. As these cells disintegrate, their contents are incorporated into a growing necrotic plaque core. ${ }^{3}$ Eventually, neovascularization, which consists of immature vessels that are fragile and prone to leakage or rupture, extends into the plaque from the adventitial vasa vasorum ${ }^{11}$ (Fig 2). Leakage or rupture can lead to hemorrhage into the plaque, which results in accumulation of blood and iron deposits. ${ }^{12}$ Intraplaque hemorrhage is regarded a key feature in the transition from stable plaque to unstable plaque that is prone to rupture. ${ }^{13}$

Histologic and Biological Features of Unstable Plaque-Histologically, the pathologist can readily differentiate between stable and unstable plaque using established criteria for vulnerability such as increased presence of inflammatory cells, smooth muscle cell loss, intraplaque hemorrhage, a lipid-rich necrotic core, and a thin fibrous cap. ${ }^{13}$ Autopsy studies indicate that, during plaque rupture, the thin fibrous cap is focally interrupted, allowing circulating blood to come in direct contact with the thrombogenic contents of the lipid-rich core. This contact triggers thrombotic occlusion of the coronary artery, which in turn leads to MI. 3,14

\section{Imaging Targets in Coronary Atherosclerosis}

Two fundamentally different imaging approaches are used in the assessment of patients with known or suspected CAD: anatomic and functional imaging. Anatomic imaging such as coronary angiography or intravascular ultrasound defines the morphologic characteristics of atherosclerotic plaques and the degree of luminal stenosis associated with atheromas. Functional imaging studies such as stress echocardiography or myocardial perfusion scintigraphy can determine whether a coronary narrowing is associated with a transstenotic pressure gradient high enough to cause myocardial ischemia.

This issue of Current Problems in Cardiology discusses the roles of noninvasive cardiac computed tomography (CCT) and cardiac magnetic resonance (CMR) for anatomic and functional imaging of $\mathrm{CAD}$, including their emerging role in molecular cardiovascular imaging and in the noninvasive characterization of atherosclerotic plaque. Recent developments in CCT and CMR offer the opportunity to noninvasively image the coronary artery lumen, the degree of coronary stenoses, and the presence of ischemia. In addition, CCT and CMR may soon also allow quantitative analysis of vessel wall structures, and visualization of plaque components and features important for the noninvasive characterization of atherosclerotic plaques. ${ }^{15,16}$ Some of these features, such as vascular calcification and coronary remodeling, can be easily 
addressed with currently available clinical computed tomographic (CT) and magnetic resonance (MR) scanners, whereas others such as neovascularization and specific molecular processes remain in the investigational realm at the time of this writing.

\section{Vascular Calcification}

The mechanisms underlying deposition of calcium hydroxyapatite at later stages of plaque development are not completely understood. They probably include changes of local tissue $\mathrm{pH}$ and the presence of proteins whose primary functions are in bone metabolism, such as osteopontin. ${ }^{17}$ The total volume of coronary artery calcium (CAC) deposits is a good indicator of overall plaque burden and of future coronary events. Therefore, CAC is used as a marker of atherosclerotic disease and of cardiovascular risk. This use of CAC imaging is discussed in detail in the clinical section on CCT later in this volume. However, localization of CAC does not correlate well with the severity or vulnerability of coronary lesions, which is particularly true in older patients. ${ }^{18}$ In fact, plaques rich in collagen and calcium are widely considered firm, rigid, and stable, whereas highly vascularized atheromas containing a core of lipids and necrotic debris are "soft" and more likely to be biologically "unstable." 11

\section{Vascular Lumen and Remodeling}

As coronary plaque enlarges, compensatory changes occur in the media that serve to maintain the coronary luminal area and thus coronary blood flow. ${ }^{19}$ The media subjacent to the plaque becomes distended and atrophied to create a larger cross-sectional vessel area (Fig 3). This mechanism is generally successful in preserving coronary blood flow until an accelerated rate of plaque growth outstrips the artery's ability to adapt adequately and in a timely manner. Typically, when the plaque occupies approximately $40 \%$ of the vessel area, noticeable impingement on the coronary lumen begins to occur. ${ }^{20}$ The phenomenon of coronary remodeling can be a significant contributor to the oft-noted discordance between the degree of coronary luminal obstruction seen on selective coronary angiography on the one hand and plaque size seen on intravascular ultrasound or histopathologic assessment on the other. The coronary artery lumen may still appear to be of normal caliber when atherosclerotic disease of the vessel wall itself is already advanced. As discussed later in this article in the section on plaque imaging, the morphology and extent of vascular remodeling may allow conclusions about the likelihood of rupture of the associated plaque.

Because plaques can cause eccentric stenoses, selective coronary angiography requires imaging in multiple projections to visualize all relevant coronary segments in a least two orthogonal views to minimize the risk of underestimating the degree of luminal narrowing. The three-dimensional (3D), volumetric nature of CCT and some forms of CMR allows reconstruction of any coronary segment from any arbitrary angle using data from only one scan. In luminographic assessment of CAD, it is important to distinguish between diameter stenosis and area stenosis. A 50\% reduction of vessel diameter $(\mathrm{mm})$ corresponds to a $75 \%$ reduction of cross-sectional vessel area $\left(\mathrm{mm}^{2}\right)$, and a $70 \%$ diameter stenosis corresponds to a $90 \%$ area stenosis. Diameter stenosis is the method currently recommended for quantifying vascular stenoses.

It is generally accepted that diameter stenoses greater than $70-75 \%$ are likely to be hemodynamically relevant, whereas diameter stenoses between 50 and $70 \%$ may not consistently reduce coronary flow reserve to a clinically significant degree. ${ }^{21,22}$ This consideration is important for the clinical evaluation of new imaging technologies such as CCT and CMR when it is necessary to define clinically significant disease, especially when comparing anatomic and functional imaging techniques. For example, landmark correlative studies between selective coronary angiography and stress testing have been divided between using 50 and $70 \%$ diameter stenosis as the anatomic definition of significant stenosis. ${ }^{23}$ This 
concern will be addressed in more detail in the sections on coronary CT angiography (CCTA) and stress MR myocardial perfusion imaging.

\section{Neovascularization}

A striking feature of vulnerable plaques is the abnormal proliferation of vasa vasorum. 11,13 ,

24 These microvessels that surround and invade the atheroma are leaky and immature and have recently been recognized as the source of plaque hemorrhage that defines the transition from stable to unstable plaque. ${ }^{5,13,14,25}$ The microvasculature supplying the atheroma is connected to the coronary artery lumen through the adventitial vasa vasorum and is therefore accessible to contrast agents. Research imaging techniques such as microscopic CT already allow a detailed look at plaque angiogenesis and the microcirculation in the vessel wall (Fig 4$)^{26}$ and in other vascular areas, ${ }^{27}$ but the routine translation of these approaches into the clinical arena will require substantial technological improvement of CCT and CMR. However, with adequate contrast agents, imaging protocols, and temporal and spatial resolution, assessment of plaque perfusion by $\mathrm{CCT}$ or CMR could become a viable imaging target in the future.

\section{Molecular Imaging Using CT and MR}

With ever-improving understanding of the vascular biology of atherosclerotic plaques, an exciting, new field has emerged at the interface between imaging, genetics, and molecular biology, with the goal of noninvasively detecting a biomolecular signature specific for vulnerable plaque. ${ }^{4,15,16}$ Molecular imaging seeks to differentiate stable from unstable plaques based on their metabolic and functional activity rather than structural or anatomic characteristics. The underlying principle is that atherosclerotic plaque exhibits biomolecular markers that may occur only at certain stages of plaque development and, therefore, are either specific for, or highly abundant in, potentially unstable lesions. These biomolecular markers include increased expression of certain cellular receptors, local production of growth factors, the presence of abnormal extracellular components, or the accumulation of circulating proteins. Some of these markers can be targeted in a highly specific fashion with molecular imaging contrast agents. Imaging specific plaque components is a particular, well-established strength of CMR but has very recently also been described for coronary CT. ${ }^{28}$

The $\alpha v \beta 3$-integrin is a general marker of angiogenesis that plays an important role in a wide variety of diseases that are characterized by neovascularization, including neoplastic disease but also CAD. The $\alpha \mathrm{v} \beta 3$-integrin is an adhesion molecule that is expressed on the luminal surface of activated endothelial cells but not on mature quiescent cells (Fig 5). ${ }^{29} \alpha \mathrm{v} \beta 3$-integrintargeted perfluorocarbon nanoparticles have successfully been used to specifically detect and characterize early-stage atherosclerosis in a hypercholesterolemic rabbit model. ${ }^{30}$

Other approaches include macrophage imaging with ultrasmall super-paramagnetic particles of iron oxide (USPIO) $)^{31}$ and imaging of thrombin activity with thrombin-sensitive nearinfrared molecular probes. ${ }^{32,33}$ New contrast materials that specifically bind to components of atherothrombotic plaque, such as fibrin-specific gadolinium conjugates, are being studied in animal models and are discussed in more detail in the section on MR plaque imaging later in this article. Eventually, such targeted contrast agents may also serve as drug delivery vehicles, allowing an approach to vascular atherosclerosis that combines diagnostic and therapeutic steps.

These biomolecular imaging approaches are very sophisticated and typically require complex labeling and amplifying of the targeted molecule and reliable and specific binding to the ligands. These requirements limit the clinical use of such techniques at the current time. Meanwhile, CCT and CMR for the evaluation of overall plaque burden, cardiovascular risk, 
and anatomic severity and functional significance of coronary artery stenoses are finding their place in the contemporary clinical practice of cardiology.

Matthew Budoff: The competency guidelines for CCT and CMR outline training requirements, and generally the timelines for competence are twice as long with CMR as CCT.

\section{Technical Aspects of Coronary and Cardiac CT and MR Imaging (MRI)}

In this volume of Current Problems in Cardiology, we discuss only those technical aspects of CMR and CCT that affect the practitioner and patient in daily practice or that the informed consumer should understand to realistically appraise the strengths and weaknesses of each technique. For details of the principles of data acquisition and image formation in CT and MR, we refer to dedicated textbooks. 34,35

At the current stage of development, CCT is easier to understand and perform than CMR. The physics principles underlying CMR are complex, and choosing the appropriate pulse sequences for specific clinical questions requires experience. Most MR pulse sequences acquire multiple parallel slices or slab-shaped volumes that do not encompass the entire heart. Therefore, a CMR examination typically consists of many image acquisitions, and experience is needed to obtain the desired views of the heart or coronary arteries. A CMR examination may last 45 minutes or longer. Conversely, a CCT scan lasts 10-15 seconds and acquires a complete 3D data set that can be reformatted in any arbitrary plane after the examination is complete. However, the number of ways in which image data acquisition in CT can be varied is limited.

\section{Computed Tomography}

How a CT study of the heart and coronary arteries is performed depends on the type of scanner being used and on the objective of the study. Dedicated CT imaging of the beating heart first became possible around 1984 with so-called electron beam CT (EBCT) scanners, which could produce images with very high temporal resolution (100 ms or less) because no moving parts were involved in the acquisition of the projection data from which the images were reconstructed. ${ }^{36}$ At that time, the acquisition of projection data for a CT image on conventional scanners with mechanically rotating gantries took several seconds, and the reconstruction of an entire study took several hours. All EBCT scanners were manufactured by one company (Imatron, Inc, San Francisco, CA), which is no longer in business.

Matthew Budoff: Imatron was acquired by General Electric, which still maintains and produces electron beam CT scanners, although demand has gone down dramatically with the development of 64-slice scanners.

Beginning in 1998, so-called multidetector CT (MDCT) scanners (also frequently referred to as multislice CT or MSCT scanners) with mechanical gantries rotating fast enough ( $\leq 500 \mathrm{~ms}$ per $360^{\circ}$ rotation) to produce images of the beating heart with no or little motion artifact have become available. ${ }^{37}$ Several manufacturers produce MDCT scanners, and MDCT technology has evolved quickly. Contemporary MDCT scanners have up to two X-ray sources mounted on gantries that rotate at a speed of as little as $330 \mathrm{~ms}$ per $360^{\circ}$ rotation and acquire 64 image slices or more (256- and 320-slice scanners are being tested) simultaneously with each gantry rotation. ${ }^{38}$ Because of these technical developments, the temporal resolution of an MDCT image of the heart can be as low as $83 \mathrm{~ms} .{ }^{38,39}$ The in-plane spatial resolution of contemporary MDCT scanners is approximately $0.45 \times 0.45 \mathrm{~mm}$, with a slice thickness of $0.6 \mathrm{~mm}$. For comparison, the temporal resolution of cinefluoroscopy in contemporary catheterization laboratories is approximately $10 \mathrm{~ms}$, and the spatial resolution is $0.2 \mathrm{~mm}$ (Fig 6). The vast majority of cardiac and coronary CT imaging studies are now performed with MDCT scanners. 
Most cardiac CT studies are performed for one of the two following purposes: either for CAC scanning or for CCTA.

Coronary Calcium Scanning-CAC scans are easily performed and require no patient preparation, and the scanning process lasts 5-10 seconds. Because calcium can be present in locations other than the coronary arteries, such as the aorta, mitral annulus, papillary muscles, myocardium, and pericardium, physician review of the resulting images is required to determine which calcifications should be included in the quantification (Fig 7 and ref. ${ }^{40}$ ). CAC is then semiautomatically quantified by radiology technicians with the use of specialized, interactive computer programs.

Matthew Budoff: While it is important to exclude thoracic and aortic valve calcification from your total coronary calcium score, there are emerging data that thoracic calcification and aortic valve calcification may carry additional prognostic value.

Several algorithms are used to quantify CAC. The best known algorithm is the Agatston score. 41 The Agatston score, developed for EBCT imaging, takes into account the areas $\left(\mathrm{mm}^{2}\right)$ occupied by calcium in each cross-sectional image of the heart, and the peak Hounsfield unit (HU), a measure of the X-ray attenuation, in each of the calcified areas. The definition of the Agatston score has conceptual disadvantages in that the numeric value of the score can vary drastically with comparatively minor variations of the peak $\mathrm{HU}$ of calcified areas. The volume score $^{42}$ and the calcium mass equivalent ${ }^{43}$ are two more recently developed approaches to quantifying $\mathrm{CAC}$, which may be less variable. However, the vast majority of studies examining the diagnostic and prognostic value of CAC have used the Agatston score, and this score remains the most widely used means of reporting CAC. CAC scores derived from MDCT imaging are often referred to as "Agatston score equivalent" because, owing to the technical differences between EBCT and MDCT scanners, there can be minor differences in the numeric value of the CAC score if the same patient is imaged sequentially with both types of scanners. 44

Because CAC scores in the range of most predictive of future cardiac events (scores > 400) are rare in the principal screening population of asymptomatic middle-aged individuals, CAC is often also reported as gender- and age-based percentile ranks derived from asymptomatic cohorts. ${ }^{45,46}$ In one study, where only $7 \%$ of 632 individuals with an average age of 52 years had an Agatston score $>400$ but $22 \%$ of cardiac events occurred in this subgroup, a percentile rank of $>75 \%$ for age and gender was a better predictor of future cardiac events than the absolute Agatston score. ${ }^{46}$ However, available data do not convincingly suggest that reporting percentile ranks rather than absolute $\mathrm{CAC}$ scores consistently increases the predictive value of CAC scanning in individual patients. ${ }^{47}$

Coronary CT Angiography-For CCTA, a modest degree of patient preparation is required. Blurred representations of the coronary arteries resulting from motion artifact can occur if the motion velocity of the coronary artery during the cardiac cycle exceeds the temporal resolution of the scanner. Because such motion artifacts can interfere with the confident interpretation of CCTAs, and because the prevalence of motion artifacts increases proportionally to the patient's heart rate during the scan, it can be advantageous to administer pharmacologic agents that reduce the heart rate before the scan. ${ }^{48}$ For CCTA with most CT scanners currently in use, a heart rate of $<60-70 \mathrm{bpm}$ is desirable, and $\beta$-receptor blocking agents are more effective than calcium-channel antagonists for heart rate reduction. The algorithms for pharmacologic heart rate control followed at Mayo Clinic in Jacksonville, FL, are shown in Fig 8 as an example. ${ }^{49}$ Patients with a high resting heart rate (70 bpm or more), who cannot safely receive pharmacologic heart rate control because of contraindications, such as second-degree or higher atrioventricular block or severe reversible obstructive airway 
disease, may not be suitable candidates for CCTA. Administering sublingual nitroglycerin to the patient immediately before the scan for vasodilation can improve the visibility of the contrast-enhanced coronary arteries on CT images. For CCTA, approximately $60-80 \mathrm{~mL}$ of iodinated contrast medium is injected, typically through a 16- or 18-gauge needle in the right forearm vein, at $4-7 \mathrm{~mL} / \mathrm{s}$, immediately followed by a saline flush at the same injection rate. The details of contrast injection will vary with practitioner preference and patient's body habitus. 50

Matthew Budoff: Most CT studies published report a 20-gauge IV with flow rates of $5 \mathrm{~mL} / \mathrm{s}$. Studies documenting the ideal flow rates for CTA of the coronaries have not yet been reported.

Once the scan is complete, several important steps take place before image interpretation, most of which require physician involvement. The continuous acquisition of projection data throughout the scan with simultaneous recording of the electrocardiogram (ECG) allows the generation of images that can represent any instance during the cardiac cycle. The first step is to decide at what time point during the cardiac cycle the images should be reconstructed. To find the time point with the least degree of motion artifact, several trial reconstructions are performed near the isovolumetric filling period in diastole. If no suitable time point can be identified during diastole, trial reconstructions near the isovolumetric contraction during systole are performed. For most patients, a reconstruction window beginning at 60,65 , or $70 \%$ or the R-to-R interval of the ECG works well; however, for many patients imaged at a heart rate near the upper limit of the desirable range, optimal image quality may be found during systole. The optimal 3D image data set can then be displayed and viewed in various ways for interpretation. 51

Safety of Cardiac CT-In addition to the risks of administering cardioactive drugs and iodinated contrast media for CCTA, performing cardiac CT exposes the patient to ionizing radiation. Radiation dose is best expressed as effective dose E, expressed in units of millisieverts (mSv), 52 which is a rough estimate of the risk of a partial body exposure to ionizing radiation, such as experienced by patients during medical imaging, expressed in a whole body exposure. The typical effective dose for a CCTA is approximately $10-20 \mathrm{mSv}$. This dose can be reduced by 30-90\% using various approaches. However, most approaches that limit radiation dose can also limit image quality, and CT imaging protocols are constantly being refined to minimize radiation dose to the patient while maintaining an image quality sufficient for confident interpretation. Some nuclear cardiology tests expose patients to a radiation dose that is similar to that of CCTA. Table 1 lists the effective radiation doses for selected nuclear imaging procedures. ${ }^{53}$

Matthew Budoff: Dose modulation (reducing the power during systole and enddiastole when images are not interpreted) has become routine (reducing radiation exposure to 6-10 mSv). Reducing the voltage (from 120 to $100 \mathrm{kV}$ ) will afford another $30-50 \%$ dose reduction that is incremental to the reduction from dose modulation and is becoming commonplace in thinner patients. Combined radiation dose reduction approaches are highly recommended in younger patient populations, which are more susceptible to radiation-induced risk.

The precise magnitude of risk of cancer induction at the levels of radiation used in medical imaging is quite controversial. ${ }^{54,55}$ Conservative estimates suggest a $0.05 \%$ ( 1 in 2000) increase in the likelihood of developing a fatal cancer from the radiation associated with a CCTA study. This increased likelihood alters the average likelihood (in the USA) of dying from cancer from about 25 to $25.05 \%$, assuming that the patient will not die from other causes (including cardiovascular disease) in the 20- to 40 -year period that a radiation-induced cancer takes to develop. 56 


\section{Cardiac and Coronary Magnetic Resonance Imaging}

MR scanners have technically matured for cardiovascular imaging and scanners with cardiovascular imaging capability are increasingly available. With CMR, two fundamentally different options are available to assess patients with suspected CAD. One option is the direct visualization of the coronary arteries (coronary magnetic resonance angiography, CMRA); the second option is the assessment of myocardial ischemia or blood flow within the myocardium, either by first-pass myocardial perfusion imaging or by dobutamine stress wall motion imaging (DSMR). CMRA provides information identical to the one invasive cardiologists are used to from selective coronary angiography (ie, location and degree of coronary artery stenosis), stress CMR provides physiological information that allows assessment of the hemodynamic consequences of stenoses similar to conventional stress testing.

CMRA is currently inferior to CCTA and not widely used. Apart from delayed myocardial enhancement studies to assess myocardial viability which have been discussed in another recent issue of Current Problems in Cardiology, ${ }^{57}$ stress imaging is the most frequent indication for CMR. Of note, the use of gadolinium chelates for CMRA and delayed enhancement imaging is off-label and not approved by the United States Food and Drug Administration.

Safety of Cardiovascular Magnetic Resonance Imaging-The static and gradient magnetic fields and radiofrequency generated in CMR can interfere with ferromagnetic or electronic devices. Therefore, MR imaging is generally considered contraindicated in patients with incompatible biometallic implants and with pacemakers or implantable cardioverterdefibrillators. 58 However, if proper precautions are taken, patients with implantable cardiac rhythm management devices can undergo MRI successfully and safely at experienced centers if absolutely needed. ${ }^{59}$ Coronary stents, ${ }^{60}$ sternal closure wires, and the majority of heart valve prostheses (the notable exception being early-generation Starr-Edwards caged ball prostheses) do not represent contraindications for CMR.

Nephrogenic systemic fibrosis (NSF) is a rare, recently described disorder seen in patients with kidney failure, particularly in patients on dialysis. ${ }^{61}$ A large proportion (> 95\%) of patients who develop NSF have had recent exposure to gadolinium. Experts estimate the risk of patients with severely impaired renal function of developing NSF after exposure to gadolinium at approximately $5 \%$. Current recommendations advise against administering gadolinium to patients with a glomerular filtration rate of $<30 \mathrm{~mL} / \mathrm{s}$. 22

Stress Imaging-The limited space in the scanner bore and the lack of MR-compatible exercise equipment mandate the use of pharmacologic stress agents for the detection of ischemia on stress CMR. Pharmacologic stress is a well-validated alternative to treadmill and bicycle exercise and is recommended in the American College of Cardiology Foundation/ American Heart Association Clinical Guidelines for patients who are unable to exercise adequately. 23

Myocardial Perfusion Imaging: Centers performing stress MR myocardial perfusion imaging use, and vendors of MRI scanners recommend, various imaging sequences and contrast agent administration protocols. The passage of gadolinium-based contrast agents through the coronary bed results in increased signal in the myocardium compared to images obtained before contrast administration. Although there is no standardized technical approach to MR myocardial perfusion imaging at this time, most centers use T1-weighted sequences for image enhancement of myocardium by gadolinium. The sequences used today for MR first-pass perfusion imaging can be implemented on a wide range of clinical 1.5-and 3-Tesla MR scanners. 
Stress Agents: Pharmacologic vasodilatation can be used to facilitate differentiation between normal and ischemic myocardium. This can be done very well with adenosine, an endogenous nucleotide, or its pro-drug dipyridamole, which is activated in the liver. Coronary arteries containing hemodynamically relevant stenoses are already dilated at rest and typically cannot dilate much further. As a result, adenosine stimulation causes an increase of blood flow in perfusion territories supplied by normal coronary arteries, whereas no change (or even a reduction) of blood flow occurs in perfusion territories supplied by stenotic coronary arteries.

The adenosine injection protocol for MR myocardial perfusion studies is very similar to that used for single-photon emission computed tomography (SPECT) myocardial perfusion studies. The administration of adenosine in the MR laboratory is very safe.

Examination Technique, Image Display, and Interpretation: Fast T1-weighted imaging during a rapid bolus injection of a low dose of a T1-shortening extracellular contrast agent (typically gadolinium chelates) produces the best results in MR perfusion imaging. Because approximately $50 \%$ of the contrast agent leaks into the interstitial space during the first pass, myocardial signal intensity during a perfusion study depends not only on myocardial blood volume and perfusion but also on the size of the extravascular compartment and the degree of capillary permeability. The contrast dose injected as a bolus varies between different centers from 0.025 to $0.15 \mathrm{mmol} / \mathrm{kg}$ of gadolinium administered via a power injector at rates ranging from 3 to $6 \mathrm{~mL} / \mathrm{s} .{ }^{63}$ The contrast agent bolus is usually followed by a 20-mL saline flush, administered at the same injection rate to keep the bolus compact. Adenosine and gadolinium should be injected through two separate 18-gauge intravenous needles for the injections.

The overall imaging procedure of adenosine stress CMR consists of the following: (1) cine wall motion imaging of the heart at rest; (2) first-pass perfusion imaging during (2a) vasodilator stress, and separated by $15 \mathrm{~min},(2 \mathrm{~b})$ at rest; and eventually (3) delayed myocardial enhancement imaging as described elsewhere. ${ }^{57}$ First-pass imaging usually takes $30-50 \mathrm{~s}$ and is performed during a prolonged breath-hold (Fig 9, ref. ${ }^{64}$ ). Wall motion is usually not assessed during adenosine stress CMR.

The upslope of signal intensity of the myocardium during the first pass of the gadolinium bolus correlates well with blood flow measured with microspheres. ${ }^{65}$ For clinical purposes, MR myocardial perfusion is at most centers assessed qualitatively by visual inspection. Myocardial regions with ischemia will show a slower and reduced inflow of gadolinium, resulting in signal intensity that is lower than in normal areas. Quantitative or semiquantitative assessment of myocardial perfusion by CMR is possible but, because it requires complex mathematical modeling, is typically performed only in the setting of research studies. To differentiate ischemia from MI, the size and location of decreased signal intensity on first-pass perfusion imaging is compared to the appearance of the respective myocardial segments on delayedenhancement images. 66

Practical Issues in Performing Stress Myocardial Perfusion MR: Volume coverage and the temporal sequence of stress and rest imaging deserve consideration as important practical issues in the performance of stress myocardial perfusion MR.

Usually, three short-axis views (near the apex, at midventricular level, and near the base), similar to those used in radionuclide myocardial perfusion imaging, are acquired for MR firstpass perfusion. This approach covers 16 of 17 myocardial segments defined in the standardized segmentation of the myocardium 67 but excludes the apex. However, the additional acquisition of a long-axis view to also visualize the apex does not improve overall diagnostic accuracy. 68 The acquisition of five rather than three short-axis views to cover a larger part of the 
myocardium also does not improve, and can in fact decrease, diagnostic accuracy compared to the standard approach. 69

It is advantageous in adenosine stress perfusion CMR to perform stress imaging before rest imaging. This is because the difference of enhancement between normal and ischemic myocardium during the first pass of a gadolinium bolus is more pronounced with the first injection than with the second injection. This, in turn, is because the gadolinium from the first injection may not be completely washed out of the myocardium by the time the second gadolinium injection is performed after 10-15 minutes. In addition, infarcted myocardium may already show delayed enhancement by the time the second perfusion scan is performed. As a result, the difference in the degree of enhancement between infarcted myocardium and normal myocardium may not be as pronounced during the second injection as during the first.

Differentiation between ischemic and infarcted myocardium can be difficult in patients who do not have regional wall motion abnormalities that match the perfusion defect. In such patients, detecting scar tissue by delayed enhancement imaging is the most reliable means to differentiate between ischemic and infarcted myocardium. Rest perfusion imaging is helpful for detecting patient-specific imaging artifacts. For example, matching perfusion defects on both stress and rest imaging in the absence of delayed myocardial enhancement most likely represent artifact.

Wall Motion Imaging: State-of-the-art MR scanners allow rapid switching of the magnetic field, resulting in very short data acquisition times. High-resolution cine imaging of the heart is possible at heart rates of up to 200 beats/min. Image quality typically remains good even if the data acquisition window is set very narrow for very fast heart rates. Today's standard pulse sequences (steady-state free precession, SSFP) provide excellent visualization of the endocardial border due to high contrast between blood and myocardium, and injection of contrast agents is not needed.

Stress Modalities: The use of vasodilator agents such as adenosine or dipyridamole for the induction of ischemic wall motion abnormalities on stress imaging has been studied. The diagnostic accuracy of vasodilator-induced regional wall motion abnormalities on MR or echocardiography stress imaging for the detection of epicardial coronary stenoses is lower than that of dobutamine stress imaging. ${ }^{70}$ Dobutamine, therefore, is the pharmacologic stress agent of choice of MR stress wall motion imaging.

The pharmacologic stress protocol for dobutamine MR imaging is the same as the standard high-dose dobutamine/atropine regimen used in stress echocardiography. Imaging is repeated in all views at each dose level. If the target heart rate is not reached at the maximum dose of dobutamine, atropine is administered. The criteria for terminating a dobutamine MR stress study are identical to those for dobutamine stress echocardiography. ${ }^{71}$

Examination Technique, Image Display, and Interpretation: MR cine imaging is usually performed with the patient in the supine position. Surface coils with several elements (usually five or six) are placed on the thorax for signal detection. SSFP in combination with parallel image acquisition and retrospective ECG gating is used. During an expiratory breath-hold of 4-6 seconds, cine loops of $>25$ phases/cardiac cycle can be acquired at heart rates of up to 200 beats $/ \mathrm{min}$. The in-plane spatial resolution of MR cine scans is approximately $1.5-2 \times 1.5-$ $2 \mathrm{~mm}$ with a slice thickness of $8 \mathrm{~mm}$.

The observer examines the MR cine images for the occurrence of new or worsening wall motion abnormalities. Cine images are displayed on the scanner console within 1 second after data acquisition and can be transferred immediately to an independent viewing station. A 
synchronized, simultaneous display at the different dobutamine dose levels is typically used for definitive interpretation of the stress study. For wall motion analysis, the myocardium is divided into 17 segments and evaluated similar to stress echo. Abnormal findings include failure of wall motion or wall thickening to increase during inotropic stimulation, or a reduction of wall motion or thickening.

Feasibility and Safety of Stress CMR: Four to 6\% of patients cannot lie in the scanner bore due to claustrophobia. Monitoring during a stress MR examination requires the same precautions and emergency equipment as any other form of stress testing. A health care provider appropriately trained in advanced cardiac life support must be present throughout the stress examination and during the recovery phase. In addition, precautions for rapid patient removal from the scanner bore must be taken. Some centers administer the pharmacologic stress agent while the patient is lying outside of the scanner bore. The blood pressure can easily be monitored with a conventional monitoring system placed outside the scanner room that is connected to the patient with an extension line, or alternatively, with special MR-compatible equipment.

For stress imaging with adenosine, potential side effects are transient and usually do not require medical intervention. ${ }^{72}$ For stress imaging with dobutamine, heart rate and rhythm should be monitored throughout the duration of the stress. The ST segments displayed on the heart monitor are nondiagnostic due to the magneto-hydrodynamic effect in the magnetic field. However, because wall motion abnormalities precede ST-segment changes in the ischemic cascade and because wall motion can readily be detected with CMR, heart rhythm monitoring during DSMR is useful and sufficient even without the ability to evaluate the ST segments during stress. In a report on the safety of high-dose dobutamine stress MR in 1000 consecutive patients, ${ }^{73}$ the safety profile of dobutamine stress MR was similar to that of dobutamine stress echocardiography.

Coronary Magnetic Resonance Angiography-Similar to what is described for CCTA above, cardiac motion must be "frozen" for CMRA to prevent blurring of the coronary arteries resulting from motion artifact. ECG triggering is used to collect all data for image reconstruction during an acquisition window of $80-150 \mathrm{~ms}$ in mid to late diastole, a time point of the cardiac cycle during which motion velocity of the coronary arteries is low.

There are two basic approaches to performing CMRA: with breath-hold or during free breathing. Breath-hold CMRA acquires targeted 3D volumes that cover one epicardial coronary artery per breath-hold. ${ }^{74}$ However, spatial resolution in MRI is related to the number of phase-encoding steps. Each phase-encoding step takes time and therefore the spatial resolution depends on how much time is available for the scan. With current technology, the data for CMRA are acquired over many heart beats, and the limited ability of many patients with CAD to hold their breath (mean, 28 seconds) ${ }^{75}$ limits the number of slices and the spatial resolution attainable by breath-hold CMRA. In addition, even during a well-executed breathhold, diaphragmatic drift of up to $1 \mathrm{~cm}$ can occur and can contribute to a blurred appearance of the coronary arteries.

Therefore, compensating for motion of the heart within the thorax due to excursion of the diaphragm must also be a consideration. This can be achieved by so-called "respiratory gating." The two most promising approaches are free-breathing CMRA of targeted 3D volumes using navigator correction algorithms to suppress breathing motion artifacts, ${ }^{76}$ and free-breathing navigator-corrected whole-heart CMRA covering the entire coronary artery tree. ${ }^{77}$ For freebreathing CMRA, a 2D radiofrequency pulse (navigator echo) is used to monitor the diaphragmatic excursions during respiration, and data for CMRA are acquired only during certain positions of the diaphragm. 
Compared to breath-hold CMRA, navigator-corrected free-breathing CMRA has the advantages of higher spatial resolution, being able to cover large 3D volumes or even the entire heart, and higher signal-to-noise ratio. However, the simultaneous use of ECG-gating and respiratory gating when using the navigator technique greatly limits the times during which data can be acquired and typically results in very long scan times of up to 3-15 minutes. The image quality can be unpredictable, in particular in patients with irregular breathing patterns.

Pulse Sequences: For all CMRA sequences, fat suppression is used to increase the contrast between the coronary artery lumen and the surrounding epicardial fat tissue. However, in images generated with standard fat-suppressed 3D gradient-echo sequences, the contrast between blood and myocardium is poor. To overcome this limitation, T2-prepared sequences that suppress the myocardial signal have been developed. 78 New SSFP sequences have even higher signal-to-noise ratio and contrast between blood and myocardium. 79

Another approach to improving the image quality of CMRA involves the administration of T1shortening contrast agents (ie, gadolinium chelates). After administration of gadolinium, socalled inversion recovery preparation can be used to improve image quality. ${ }^{80}$ The inversion time is set such that the signal from the myocardium is minimized. This approach also improves the contrast-to-noise ratio between the coronary artery lumen and the myocardium. As noted previously, the use of gadolinium for CMR is not United States Food and Drug Administration approved.

Contrast Agents: The currently available gadolinium chelates remain in the intravascular blood pool for only a brief period of time and rapidly extravasate into the interstitial space. Therefore, most of these contrast agents improve the image quality of 3D CMRA only for a short time after intravenous application.

More recently, MR contrast agents with prolonged intravascular dwelling time have been developed. These "intravascular" contrast agents include gadolinium molecules bound to manufactured macromolecules, such as P792 (Vistarem; Guerbet, Aulnaysous-Bois, France) and Gadomer (Vasovist; Bayer Schering Pharma, Berlin-Wedding, Germany), and gadolinium molecules with high affinity for albumin, such as Vasovist (Bayer Schering Pharma) or B-22956 (Bracco, Milan, Italy). Vasovist has already been approved for MR angiography in Europe. The use of these compounds substantially improved contrast-to-noise ratio of CMRA in preclinical studies, and in healthy volunteers and patients, 81,82 several studies comparing CMRA performed using intravascular contrast agents with MRA without contrast enhancement demonstrated that the higher signal-to-noise ratio of the contrast-enhanced sequences significantly improved diagnostic accuracy. ${ }^{82-84}$

Matthew Budoff: New warnings related to gadolinium use in renal insufficiency and renal failure related to nephrogenic systemic fibrosis makes this enhancement agent contraindicated in those persons with stage 4 or 5 renal dysfunction.

Plaque Imaging -Even more so than for CMRA performed to evaluate coronary luminal dimensions, MRI of the coronary vessel wall requires high spatial resolution and image contrast. ECG triggering and navigator-gating to compensate for diaphragmatic motion are routinely used for this type of study. Turbo spin-echo sequences, combined with dark blood preparation and fat saturation, maximize the signal from the coronary artery wall and the contrast between the coronary artery wall on the one hand and the coronary artery lumen and the epicardial fat tissue, which appears dark on this type of sequence, on the other. Imaging at higher field strength (3 T) and radial k-space sampling, which increase signal-to-noise ratio and reduce motion artifacts, can further improve image quality. Most recently, ultrahigh field 7.0 T MRI of human iliac arteries in vitro has allowed reliable detection of plaques $<1 \mathrm{~mm}$ in 
size and accurate visualization and quantitative assessment of vessel wall composition compared to histology. 85

The ability to identify on MRI coronary artery lesions at risk of rupture by enhancing the entire plaque or specific plaque components would be of great clinical interest. However, plaque enhancement by conventional gadolinium-based contrast agents may be a nonspecific finding. In addition, the apparent increased enhancement of thickened vessel wall compared to normal vessel wall can at least in part be explained by a reduction of partial volume effects. Such a reduction occurs when the dimensions of a structure of interest (ie, vessel wall thickness) increase in the presence of limited, fixed spatial resolution (such as present in CMR). Gadofluorine, another research compound developed for vessel wall imaging, selectively enhances artherosclerotic plaques, whereas normal aortic wall does not enhance. ${ }^{86}$ Although the mechanism of enhancement is not completely understood, this contrast agent may in the future help with detection of plaque and the visualization of plaque growth in vivo.

More recently, investigational compounds that selectively accumulate in atherosclerotic plaques or bind to plaque components and can be visualized on MRI have shown interesting results in preclinical studies. For example, USPIO injected at high doses are taken up by phagocytosis by the macrophages abundantly present in atherosclerotic plaque. The accumulated USPIOs can be detected by MRI due to the susceptibility artifact they cause (Fig 10). ${ }^{87}$ This approach indirectly indicates the presence of macrophages, and by implication inflammatory processes likely associated with biological "instability," within the vessel wall. Another attractive concept is the use of paramagnetic nanoparticles, which actively target specific components of plaque or markers of plaque physiology associated with increased risk of rupture 88 as discussed in the introductory sections of this article. Contrast agents that selectively bind to the fibrin that is present on the surface of plaques near fissures that may precede rupture (Fig 11) ${ }^{89}$ or other markers of vascular injury such as tissue factor 90 may eventually evolve into clinically useful approaches to detecting subclinical, or confirming clinically apparent, rupture of atherosclerotic plaque.

\section{Clinical Applications of Cardiac CT and MR}

CMR and CCT have evolved from investigational techniques with "potential" in imaging modalities that can be used in routine clinical decision-making. At the current stage of development, CMR and CCT have different but synergistic strengths. The main clinical uses of CT in the clinical arena are CAC scanning and CCTA. The main clinical uses of CMR are stress imaging and delayed myocardial enhancement. Delayed myocardial enhancement by MRI has been discussed in detail in a recent issue of Current Problems in Cardiology. 57 Therefore, only the data supporting CAC scanning, CCTA, CMR stress imaging, and, as a clinically important but currently largely investigational approach, plaque imaging are presented and discussed in following paragraphs. The current recommendations endorsed by professional societies of cardiologists and radiologists for the clinical use of cardiovascular CT and CMR are summarized in the "Summary and Recommendations" section.

\section{Coronary Calcium Scanning}

From an epidemiology perspective, the prevalence and quantity of CAC increases proportionally to patient age, more strongly so after the age of 50 years in men and 60 years in women than before. ${ }^{45}$ Until the age of 65-70 years, the prevalence of CAC in women at any given age is lower than that in men of the same age, and roughly equal to that of men who are 10 years younger. After the age of 65 years, the prevalence of CAC at any given age in women and men is roughly equal. Above the age of 70 years, almost $100 \%$ of men and women have CAC. ${ }^{91}$ However, at any given age, the quantity of CAC is lower in women than in men. 
Almost all patients with CAD have CAC, and patients without CAC almost never have clinically relevant amounts of coronary plaque. ${ }^{92,93}$

Quantification of coronary calcium can serve the two following related purposes: it can help estimate the probability of "significant" coronary stenoses being present (typically in patients with chest pain) and it is used to estimate the risk of cardiovascular events ("screening" in asymptomatic patients).

Diagnosis of Coronary Stenoses-The use of CAC to estimate the probability of CAD being the cause of chest pain in symptomatic patients relies on relatively straightforward principles. The area of CAC detected on CT is linearly proportional to, but represents only approximately $20 \%$ of, the coronary artery plaque area determined on histology. ${ }^{94}$ Therefore, the higher the CAC score, the more coronary artery plaque is present. However, because of the process of arterial remodeling discussed in the introductory section of this article, large amounts of plaque, and hence CAC on CT scanning, may be present before luminal narrowing occurs. The ability to remodel may vary between individuals. As a result, the correlation between CAC and the degree of coronary artery stenosis determined on histology is weak, 95 and the location of CAC does not predict the location of coronary artery stenoses.

From the fact that CAC can be present in obstructive as well as in nonobstructive lesions, it follows that using the presence of CAC as a predictor of "significant" CAD in symptomatic patients is highly sensitive (96\%) but not very specific (40\%). ${ }^{92}$ Using a set threshold of CAC quantity (eg, an Agatston score of > 80) to predict high-grade stenoses in a study of 1851 patients referred for invasive selective coronary angiography increased the specificity to $72 \%$ but decreased sensitivity to $79 \% .{ }^{92}$ Given the variations in CAC prevalence discussed above, the appropriate CAC threshold to predict coronary stenoses with highest sensitivity and specificity may vary with age and gender. In a study of 1764 patients, 93 the CAC thresholds that identified $90 \%$ of patients with high-grade CAD and $95 \%$ of patients without high-grade CAD increased with age and were, at any given age, lower in women than in men (Fig 12).

Among patients with low CAC scores in a study of 1195 patients who also underwent myocardial SPECT perfusion imaging, $<2 \%$ of those with a CAC score $<100$ had an abnormal study. ${ }^{96}$ Absence of CAC is rare in patients with significant CAD: in a study of 2155 patients referred for angiography, only $0.7 \%$ of 872 men and $0.02 \%$ of 383 women with high-grade CAD had no CAC on CT. ${ }^{97}$ The phenomenon of significant CAD in the absence of CAC appears to be more prevalent in patients younger than than 45 years than in patients older than that.

The high sensitivity of CAC for the prediction of CAD has also been applied to the clinical scenarios of unexplained left ventricular (LV) dysfunction and the triage of patients symptomatic with chest pain in the emergency department (ED). In 125 patients with an ejection fraction $<40 \%$ who underwent CAC scanning and invasive, selective coronary angiography, CAC was present in 71 of 72 patients with ischemic cardiomyopathy (sensitivity, $99 \%$ ). Only $17 \%$ of patients with idiopathic, dilated cardiomyopathy had any CAC. The mean CAC score in patients with ischemic cardiomyopathy was 798, and that of patients with idiopathic, dilated cardiomyopathy was 17 among 134 patients who presented to an ED with chest pain, a calcium score of 0 identified patients without abnormalities on conventional cardiac testing with a negative predictive value (NPV) of $100 \% .98$

Prognostication in Asymptomatic Individuals-The use of CAC to predict future cardiac events requires consideration of the relationship between CAC and histologically unstable plaque that is likely to rupture. This relationship was briefly discussed in the introductory sections of this article. 
The presence of calcification alters the mechanical properties of plaque. A plaque with a heavily calcified cap is markedly stiffer than highly cellular plaque. ${ }^{99}$ Some studies show that calcified areas are unlikely to be the site of plaque rupture, ${ }^{100}$ but another theory holds that plaque rupture might occur as the result of shear stress between calcified and noncalcified portions, similar to the mechanism that can cause plaque rupture or dissection during unstented angioplasty. ${ }^{101}$ However, the most likely explanation for the association of CAC with increased risk of future cardiovascular events is colocalization of calcified, stable plaque and noncalcified, "soft" plaque, which seem to coexist in fairly stable ratios. 94,102 Hence, the more CAC is seen on CT, the more calcified plaque and, by implication, noncalcified, potentially unstable plaque, is present. ${ }^{47}$

Early studies of the relationship between CAC and patient outcomes have been criticized for a multitude of methodologic shortcomings. ${ }^{103,104}$ A meta-analysis of six recent studies selected for their methodologic rigor and quality $105-110$ examined the relationship between CAC and 395 hard cardiovascular events in 27,622 previously asymptomatic patients (Fig 13). Patients with any detectable CAC had a more than fourfold risk of events. Conversely, the 3to 5-year rate of cardiac events in patients without any CAC was very low at $0.4 \%$.

This meta-analysis also suggested that the risk of cardiovascular events increases with the quantity of CAC. With a CAC score of 1-112, the risk of cardiac events increased 1.9-fold, whereas a CAC score of > 1000 increased the risk 10.8 -fold ( $3-5$ years rate of events, $7.1 \%$ ). Some of these studies also found that the predictive power of CAC is independent of the predictive power of traditional or nontraditional historical and measured risk factors including C-reactive protein, family history of premature CAD, and obesity. $105,109,110$ Secondary analyses further suggest that the potential value of CAC scoring is greatest in patients with intermediate (10-20\% over 10 years) risk of cardiac events. For example, among patients with intermediate risk based on Framingham criteria, the annual rate of hard cardiac events was $0.4 \%$ in patients with a CAC score of $<100$, but $2.4 \%$ in patients with a CAC score $>400$. Thus, patients with intermediate risk based on Framingham criteria alone could be considered to actually be at high risk for cardiac events (> $20 \%$ over 10 years) if their CAC score is $>400$.

Consideration has also been given to the possibility that the progression of CAC, perhaps as a marker of disease activity, could further refine the predictive power of CAC scanning or be used to monitor the efficacy of risk factor management. The annualized progression of CAC is dependent on the CAC score at baseline. ${ }^{111}$ The annualized progression differs between patients without and with cardiovascular risk factors 112,113 and it is approximately twice as high in patients who suffered a cardiac event than in patients who did not. ${ }^{114-116}$ However, three randomized, prospective trials that examined the effects of varying levels of cholesterinlowering therapy on $\mathrm{CAC}^{117,118}$ did not find lower rates of progression with higher intensities of therapy. Among the potential limitations of these studies that might have contributed to the negative findings are the use of strategies for risk factor management, that are no longer contemporary and the short follow-up (1 year).

\section{Noninvasive Coronary Angiography}

Invasive catheter coronary artery angiography must still be considered the standard of reference for the anatomic assessment of coronary artery disease. Neither CMRA nor CCTA, even including the latest technical developments such as dual source CT, have the high spatial and temporal resolution of invasive, selective coronary angiography. However, the rate of major complications of approximately $0.3-1.1 \%$, the exposure to ionizing radiation and iodinated contrast medium, and the high cost of invasive, selective coronary angiography have promoted the development of alternative, noninvasive strategies of directly visualizing the coronary artery lumen. 
Owing to relatively recent hardware and software developments, noninvasive coronary imaging with MDCT has evolved from an investigational tool to a stable, potentially clinically useful imaging modality. ${ }^{119,120}$ Initial single-center experience at specialized centers has provided an understanding of the strengths and limitations in comparison to existing diagnostic modalities.

With the availability of ultrafast imaging sequences, CMRA has become possible. The main hindrances to the routine clinical use of CMRA include image artifacts resulting from cardiac and respiratory motion, the relatively low spatial resolution of about $1 \mathrm{~mm}^{3}$, and the low signalto-noise ratio.

\section{Coronary Computed Tomographic Angiography}

Test Performance and Appropriate Patient Populations: Angiographic identification and quantification of coronary luminal stenoses often form the basis for treatment decisions in patients with symptomatic CAD. However, the anatomic severity of luminal stenosis is only a marker for its functional effect on myocardial blood flow. ${ }^{121-123}$ Animal and human studies demonstrate that coronary flow reserve, a measure of myocardial blood flow regulation, remains normal until diameter stenosis approaches $75 \%$ and dramatically decreases between 75 and $95 \%$ diameter stenosis. Given a typical caliber the coronary arteries of $<3.5-4.0 \mathrm{~mm}$, the angiographic difference between the luminal dimensions of lesions that affect coronary flow reserve minimally or severely may be only a fraction of a millimeter. Further, given the limitations of spatial resolution of all clinically available coronary imaging modalities (eg, cinefluoroscopy in contemporary coronary catheterization laboratories has a spatial resolution of approximately $0.2 \mathrm{~mm}$ and 64-slice MDCT scanners, approximately $0.45 \mathrm{~mm}$ ), these small anatomic differences can be difficult or impossible to discern.

The performance characteristics of CCTA do not support imaging of populations with high and low pretest probability of CAD. In high-risk patients, especially those with known CAD, many of the advanced atherosclerotic plaques are calcified. Dense calcification is associated with a characteristic "blooming" artifact on CT images. The blooming appearance of calcium is related to the principles that govern the reconstruction of projection data into cross-sectional images. The volumetric 3D CT data set consists of small volumetric elements (voxels), and the grayscale value of each voxel is defined by the mean of all HU contained in the voxel. Therefore, even a small amount of calcium (HU of, eg, 1000) in a given voxel of otherwise noncalcified atherosclerotic plaque ( $\mathrm{HU}$ of, eg, 40 ) increases the mean grayscale of the entire voxel, giving the appearance of a much larger calcification (Fig 14). Calcium blooming leads to overestimation of lesion severity and often precludes assessment of densely calcified segments altogether (Fig 15).

From a clinical perspective, a positive test result in a patient with high-pretest likelihood would only confirm the need for invasive imaging. Conversely, imaging of low-risk populations to exclude asymptomatic stenoses and subclinical atherosclerotic plaque accumulation is also not a meaningful approach because of the radiation exposure associated with current CT technology and the lack of data guiding translation of abnormal findings into management strategies that will improve the outcomes of such patients.

CCTA appears appropriate in selected clinical scenarios in intermediate-risk populations, particularly in patients with chest pain symptoms. In these patients, CCTA may not only allow avoiding further, more invasive testing if it clearly shows absence of significant luminal stenosis but also provide prognostic information by assessing he extent of atherosclerotic plaque burden. 
Diagnostic Performance of CCTA: The current technical limitations of CCTA are reflected in the results of comparative studies that have compared CCTA for the assessment of focal luminal stenosis to selective, invasive angiography. ${ }^{124-127}$ A meta-analysis of 29 studies published between 2002 and 2006 that used MDCT with 16 or more detector rows and included more than 1500 patients in total has examined the diagnostic accuracy of CCTA for detecting coronary artery stenoses. ${ }^{124}$ Eighteen of the studies used 16-slice MDCT, 1 study each used 32-slice or 40-slice MDCT, and 9 studies used 64-slice MDCT. The use of premedication with beta-receptor blocking agents and nitroglycerin was not consistent among the studies. Twentyseven studies reported comparisons of individual coronary segments between the two imaging techniques (22,798 total segments). The per-segment sensitivity was $81 \%$ (95\% confidence interval (CI) 72-89\%), specificity 93\% (90-97\%), positive-predictive value (PPV) 67.8\% (57.6-78.0\%), and NPV 96.5\% (94.7-98.3\%) (Fig 16). Twenty-one of the studies reported per-patient analysis (1570 patients) and cumulatively showed a sensitivity of 96\% (94-98\%), specificity of 74\% (65-84\%), PPV of 83\% (76-90\%), and NPV of 94\% (89-99\%) (Fig 17). The meta-analysis found a trend toward improvement of sensitivity and specificity with newer generations of scanner technology. This trend was confirmed in a later meta-analysis that included a higher proportion of 64-slice CCTA studies. ${ }^{128}$

Several important aspects about these pooled CT data should be kept in mind. PPV and NPV are dependent on the prevalence of disease in the population. The prevalence of CAD in these meta-analyses was $>60 \%$. It is conceivable that in populations with lower prevalence of CAD the accuracy of CCTA will be different, with a higher frequency of false-negative and falsepositive test results. Because CTA appears most appropriate in patients at intermediate risk of $\mathrm{CAD}$, it will be critical to perform validation studies predominantly in such populations. It is also important to understand the implications of performing analyses by coronary segments versus analyses by patient. ${ }^{126}$ Segment-based analysis is most useful if a diagnostic test is used to make management decisions on individual coronary artery lesions. This is typically the case in patients with established CAD. In contrast, patient-based analysis is most appropriate if the test is used to determine whether a patient has CAD.

As another important consideration, the meta-analysis reported above describes pooled results from experienced single centers. It is unclear whether the results at centers with less experience would be similar. One early multicenter trial that examined the ability of 16-row MDCT to detect coronary artery stenoses $>50 \%$ in all segments with a diameter $>2 \mathrm{~mm}^{125}$ included 238 patients referred for elective coronary angiography. Of those, 51 patients were excluded because of Agatston scores $>600(n=37)$ or for other reasons $(n=14)$. Of the remaining 187 patients (1635 segments), $29 \%$ of 1629 segments that contained no stents (63 patients) could not be evaluated due to respiratory motion artifact $(n=90)$, cardiac motion artifact $(n=211)$, excessive calcification $(n=23)$, poor opacification $(n=247)$, and small vessel size $(n=147)$. After censoring all segments that could not be evaluated as "abnormal" (ie, containing a $>50 \%$ stenosis), sensitivity in per-segment analysis was $89 \%$, specificity $65 \%$, PPV $13 \%$, and NPV 99\%. In per-patient analysis, sensitivity was $98 \%$, specificity 54\%, PPV 50\%, and NPV 99\%.

Comparing these results from a multicenter trial of $\mathrm{CCTA}^{125}$ to the meta-analysis of CCTA discussed previously 124 is informative. Sensitivity and NPV were similar, but specificity and PPV were poor. Although segments with extensive calcification were excluded a priori, there remained a high proportion of coronary segments that could not be evaluated for reasons other than CAC. Censoring of these segments as "abnormal" increased NPV but decreased specificity and PPV. These findings are not surprising in the context of the current technical limitations of CCTA discussed above. Advances in scanner technology (acquisition of $>64$ slices per gantry rotation, faster gantry rotation, dual source systems) will likely improve the diagnostic accuracy, but the clinical value for the assessment of stenosis severity in patients with advanced focal, high-grade lesions may well remain limited. ${ }^{127}$ Similar to stress-testing, 
noninvasive angiography will likely be most appropriate in patients with no more than intermediate pretest probability of high-grade coronary artery stenoses. In these patients, the diagnostic goal is the exclusion of significant coronary stenoses and the assessment of future cardiovascular risk. It seems therefore more important to compare CCTA to other noninvasive tests, especially stress testing, rather than to invasive, selective coronary angiography.

A recent study compared 16-slice MDCT and exercise ECG for the diagnosis of CAD, using invasive, selective coronary angiography as the reference standard. ${ }^{129}$ A consecutive cohort of 80 patients with suspected CAD was examined, following standard protocols. Both the sensitivity and the specificity of MDCT (91\% [40 of 44 patients, 95\% CI 78-97\%] and 83\% [30 of 36 patients, 95\% CI 67-94\%]) were significantly higher $(P=0.039$ and $P<0.001)$ than those for stress-testing (73\% [32 of 44 patients, $95 \%$ CI 57-85\%] and 31\% [11 of 36 patients, $95 \%$ CI 16-48\%]). The rate of nondiagnostic examinations was not significantly $(P=0.078)$ different between MDCT and stress-testing (8\% [6 of 80 patients, 95\% CI 3-16\%] versus 19\% [15 of 80 patients, $95 \%$ CI 11-29\%]). Another study examined, in 62 patients with typical angina pectoris, the diagnostic value of exercise ECG and 16-slice multidetector CCTA, alone and in combination, to predict $\geq 50 \%$ diameter stenoses detected on invasive, selective coronary angiography in any coronary artery branch with a caliber of $\geq 2 \mathrm{~mm} .{ }^{130}$ The sensitivity of exercise ECG was $78 \%$; specificity was $67 \%$, and PPV and NPV were 89 and $47 \%$, respectively. The sensitivity of CCTA was $100 \%$; specificity was $87 \%$, and PPV and NPV were 96 and $100 \%$, respectively. An abnormal CCTA increased the post-test probability of significant CAD after a positive exercise ECG from 89 to $99 \%$ and after a negative exercise ECG from 58 to $91 \%$. A normal CCTA reduced the post-test probability of significant CAD after a negative or a positive exercise ECG to $0 \%$.

It is important to recognize that, when using CCTA as a first-line test for diagnosing CAD as an alternative to stress testing, one foregoes important information from stress testing that conveys diagnostic and prognostic information, including electrocardiographic,

hemodynamic, exercise capacity-related, and symptomatic criteria. On the other hand, similar to other imaging modalities that assess atherosclerotic burden, CCTA may also have prognostic value that is yet to be examined and understood in detail. ${ }^{131}$ This concept is discussed in the section on plaque imaging by CT below.

Matthew Budoff: The ability to see plaque burden in addition to stenosis severity potentially makes cardiac CT a robust prognostication tool. In three studies, calcium scanning predicted future cardiovascular events better than either stress nuclear testing or stress echocardiography. Given the higher diagnostic accuracy of CTA than stress imaging, increasing numbers of physicians are turning to this tool as a first-line test for low- to intermediate-probability patients. Further head-to-head studies are currently underway.

Future studies will need to examine the clinical value of CCTA for specific indications in the context of standard diagnostic algorithms. For example, patients with unexplained LV dysfunction 132 or patients who need assessment of the coronary arteries before noncoronary cardiac surgery 133 could exploit the high NPV of CCTA (Fig 18). CCTA may also prove useful in situations where the diagnostic accuracy of conventional stress testing is reduced (eg, in the presence of left bundle branch block). ${ }^{134}$ Accumulating evidence-based data will allow defining the role of CCTA in the management of patients in certain clinical scenarios or at various stages of CAD.

The use of CCT as a cost-effective and efficient means of assessing patients with acute chest pain in the ED by simultaneously addressing the diagnostic possibilities of CAD, pulmonary embolism, and aortic dissection ("triple rule-out") is the subject of much interest and active investigation. ${ }^{135,136} \mathrm{As}$ an important challenge in this approach, it can be difficult to achieve 
satisfactory contrast opacification in the coronary arteries, pulmonary arteries, and aorta simultaneously. Contrast injection protocols that are specific to this application of CCT, and that differ from the contrast injection protocols commonly used for CCTA, have been developed. ${ }^{137}$

Coronary Magnetic Resonance Angiography (CMRA)—Two large studies, each including more than 100 patients, have compared free-breathing navigator-gated CMRA without gadolinium enhancement to invasive, selective coronary angiography and have reported high sensitivity for the detection of relevant coronary CAD. ${ }^{138,139}$

In a multicenter trial including 109 patients, images of diagnostic quality could be obtained in 103 patients. ${ }^{138}$ The sensitivity for detecting $\geq 50 \%$ diameter coronary artery stenoses was $93 \%$ but the specificity was low at $42 \%$. However, for the clinically most relevant findings, including left main coronary artery stenoses and three-vessel disease, the sensitivity was $100 \%$ and specificity was $85 \%$. Sommer et al ${ }^{139}$ included 107 patients in a single-center study that used a comparable CMRA approach to detect coronary artery diameter stenoses $\geq 50 \%$ found on selective, invasive coronary angiography. Thirty patients (28\%) were excluded because of poor image quality. In the remaining 77 patients, the sensitivity was $88 \%$ and specificity was $91 \%$.

Comparisons of CCTA and CMRA-Three recent studies support the notion that at the current stage of technical development, CCTA is more stable than CMRA for the detection of coronary artery stenoses. A meta-analysis of 51 studies ${ }^{140}$ that each examined either CCTA $(\mathrm{n}=24)$ or CMRA $(\mathrm{n}=28)$ reported a significantly higher sensitivity for CCTA $(85 \%$; $95 \%$ CI, 86-88\%) than for CMRA (72\%; 95\% CI, 69-75\%). Specificity was also significantly higher for CCTA (95\%; 95\% CI, 95-95\%) than for CMRA (87\%, 95\% CI, 86-88\%). Of note, the definition of "significant" coronary stenoses varied between 50 and $70 \%$ among the studies included in this meta-analysis.

Two recent studies compared CCTA and CMRA head to head in the same patients, using invasive, selective coronary angiography as a reference. One study of 52 patients ${ }^{141}$ reported similar sensitivity for CCTA (per patient, 92\%; per segment, 77\%) and CMRA (per patient, $88 \%$; per segment, $75 \%$ ). Specificity was also similar between the two imaging modalities: CCTA (per patient, 67\%; per segment, 79\%) and CMRA (per patient, 50\%; per segment, 82\%) (Fig 19). However, a larger study in 108 patients ${ }^{142}$ reported results similar to the aforementioned meta-analysis with significantly higher per-patient sensitivity for CCTA (92\%) than for CMRA (74\%). Of note, this study used a CMRA technique that had previously been shown to yield suboptimal results. ${ }^{143}$

Matthew Budoff: Due to lower spatial resolution and thicker slice thickness, MRCA is limited to the proximal vessels, and diagnostic accuracy in the only multicenter trial was only $72 \%$, far lower than the $90 \%$ accuracy seen in the 64-slice CTCA multicenter trial data. Since the coronary artery path is still easily evaluated, coronary anomaly evaluation remains a strong application of MRCA.

\section{Stress Imaging}

Although establishing the presence of myocardial ischemia might seem less intuitive than directly visualizing coronary artery stenoses, determining the functional consequences of CAD rather than quantifying the severity of luminal stenosis has several advantages. As discussed in other sections of this issue of Current Problems in Cardiology, the relationship between luminal narrowing and reduction of coronary blood flow is very complex and determined not only by severity, but also morphology and length of stenoses. $7,21,22$ Selective coronary angiography is by many considered the reference standard for evaluating the presence and 
severity of CAD. Perceived or real discrepancies between anatomic angiographic and functional stress imaging do not necessarily indicate a failure of the latter; instead, they indicate the complexity of the relationship between anatomical and physiological conditions. Several specific situations can lead to abnormal perfusion studies in the absence of stenoses in the epicardial coronary arteries. These situations, listed in Table 2 , are generally combined under the term "microvascular disease."144

Conversely, perfusion studies may be truly negative even in the presence of coronary stenoses. The importance of functional imaging is demonstrated by the following two points: first, as a practical clinical matter, patients' symptoms and prognosis are closely related to the presence and severity of myocardial ischemia. Second, revascularization of "borderline coronary stenoses (50-60\% diameter in locations other than the left main coronary artery)" identified on selective coronary angiography in symptomatic patients is recommended in current clinical guidelines 145,146 only if there is "demonstrable ischemia on noninvasive testing."

In general, stress MR first-pass myocardial perfusion imaging and DSMR are considered interchangeable in their use for the diagnosis of myocardial ischemia, even though differences may exist. Both DSMR and myocardial perfusion imaging are based on the fundamental concept that a decrease in myocardial perfusion, represented by perfusion defects on appropriate imaging techniques, is the first in a sequence of events that is known as the ischemic cascade. Regional wall motion abnormalities are the next step in this cascade, followed much later by ECG changes, and then by angina pain.

Myocardial Perfusion Imaging-SPECT and positron emission tomography (PET) currently dominate myocardial perfusion imaging in clinical practice because of their long track record and their well-established evidence base of diagnostic accuracy and prognostic relevance. However, these techniques have important limitations, such as the need to administer radioactive tracers, the occurrence of attenuation artifacts in SPECT which can limit diagnostic accuracy, and the limited availability of PET. PET is considered the reference standard for myocardial perfusion imaging.

Assessment of myocardial blood flow, viability, and scar tissue with CMR is well validated. The use of CT for myocardial perfusion imaging is still investigational.

Magnetic Resonance: MR first-pass perfusion imaging has seen rapid development over the past years and has shown promising results in initial multicenter trials. 63,147 One important advantage of MR perfusion imaging is its ability to visualize subendocardial perfusion defects, which is not possible with PET or SPECT due to their limited spatial resolution.

Despite continuing improvements of imaging methods, the image quality of MR myocardial perfusion studies can be variable. Apart from motion artifacts related to breathing, dark subendocardial artifacts reminiscent of perfusion defects are frequently observed in normal subjects. This appearance is most likely related to "susceptibility" resulting from the high difference in signal intensity between the gadolinium-enhanced blood pool in the LV and the adjacent subendocardium. Because the concentration of contrast medium in the blood pool declines rapidly, these artifacts diminish quickly within a few seconds after the arrival of contrast medium in the LV cavity. True perfusion defects (Fig 20), on the other hand, usually persist beyond the point of peak blood pool enhancement. This difference in duration usually allows the important differentiation between susceptibility artifact and true subendocardial hypoperfusion.

In animal studies, MR first-pass perfusion imaging correlates well with myocardial perfusion measured with microspheres and is superior to SPECT because of its higher spatial resolution. 
148 Even though this superiority in the experimental setting has to date not been confirmed in clinical prospective multicenter trials, a large body of data suggests that MR first-pass perfusion is, at least, noninferior to SPECT. In addition, MR myocardial perfusion imaging has the advantages of not exposing patients to ionizing radiation, being performed in a single 45-minute examination, and yielding a large amount of useful clinical data in addition to information on myocardial perfusion, such as precise measurements of LV function and mass, and assessment of valvular function and myocardial scar. A selection of reports on myocardial perfusion imaging $63,66,69,70,147,149-152$ is listed in Table 3.

To date, less data are available on the prognostic value of MR myocardial perfusion imaging than for SPECT imaging, which has been in use for more than 30 years. However, initial reports on the prognostic value of MR first-pass perfusion imaging have been published. A recent study 153 of adenosine-stress perfusion MRI in patients with chest pain, negative troponin I test results, and nondiagnostic ECG findings showed a sensitivity of $100 \%$ and a specificity of $93 \%$ for the prediction of 20 future adverse cardiac outcomes in 135 patients. Patients with normal stress myocardial perfusion had no adverse cardiac outcomes during 1 year of followup. In another study 154 that followed 513 patients over a median of 2.3 years during which 19 adverse cardiac events occurred, a negative perfusion study was associated with cumulative event rates of $0.7,0.7$, and $2.3 \%$ for the first 3 years. This event rate was significantly lower than in patients with abnormalities on the MR myocardial perfusion study $(6.2,12.2$, and $16.3 \%$ cumulative event rate).

Matthew Budoff: The calcium score has been demonstrated to have significant independent and incremental prognostic information to traditional risk factors in every study published to date. This has led the American College of Cardiology, the American Heart Association, and the National Cholesterol Education Panel Guidelines to all recommend consideration of the use of calcium scoring in the intermediate-risk population for further risk stratification.

Computed Tomography: The potential of CT myocardial perfusion imaging has been described with EBCT, ${ }^{155}$ and more recently in rest and stress perfusion animal models with MDCT. ${ }^{156}$ A potential advantage of MDCT perfusion imaging compared to MRI is that myocardial enhancement following injection of iodinated contrast agents is directly proportional to myocardial perfusion. ${ }^{157}$ Therefore, the complex mathematical modeling needed for quantitative assessment of myocardial perfusion by MRI is not needed for CT. Nikolaou et al 158 evaluated in 30 patients a "snapshot" (single time point) myocardial perfusion 16-slice MDCT protocol that was adapted from a coronary artery imaging protocol. The investigators detected 10 of 11 MIs (sensitivity, 93\%) that were confirmed by delayed enhancement MRI as the standard of reference. However, the sensitivity for predicting hypoperfusion diagnosed on MR myocardial first-pass perfusion imaging was only $50 \%$. Myocardial perfusion assessment by CT requires sequential imaging at several anatomic levels of the LV during the first pass of a bolus of iodinated contrast medium, and concerns about the radiation dose associated with this approach, along with the limited temporal resolution of contemporary CT scanners, have prevented large-scale clinical research in this field. With the advent of dual source $\mathrm{CT}$ and $\mathrm{CT}$ scanners that can image the entire heart in one gantry rotation, myocardial perfusion imaging may become easier.

On routine coronary MDCT, areas of MI have significantly lower CT attenuation values than normal myocardium, and chronic MIs have lower attenuation values than recent ones. ${ }^{159} \mathrm{In}$ recent preclinical studies, acute infarction, chronic scar, and microvascular obstruction could be characterized and differentiated from each other by contrast-enhanced MDCT with delayed enhancement patterns similar to those seen on CMR. ${ }^{160,161}$ MDCT imaging approximately 5 min after injection of iodinated contrast agents shows significantly higher $\mathrm{HU}$ values in acute 
and chronic MIs than in normal myocardium. Conversely, areas of microvascular obstruction within an acute MI demonstrate significantly lower HU values compared to normal myocardium. ${ }^{160}$ The additional radiation exposure of MDCT delayed scar assessment is a concern, but end-diastolic imaging with prospective ECG triggering may be used to keep the additional exposure to a minimum.

Wall Motion Imaging with CMR-Wall motion abnormalities occur later in the ischemic cascade than perfusion deficits. Consequently, they are less sensitive but more specific for the detection of coronary artery stenoses than perfusion defects. ${ }^{70}$

Wall motion imaging with CMR is technically less challenging than CMR perfusion imaging. Similar to stress echocardiography, stress MRI wall motion imaging is not only useful for the diagnosis of ischemia but may also help identify dysfunctional but viable myocardium and define patients' prognosis. CMR wall motion imaging may be superior to CMR perfusion imaging in patients with complex cardiac problems, such as after MI or bypass surgery, or those with reduced coronary flow reserve due to microvascular disease. Nonetheless, MR wall motion imaging is currently not widely used in the USA. Wall motion imaging with CCT has been described but is not widely used, mostly because of the cumulative radiation dose incurred by repeat imaging at various levels of stress.

Diagnosis of Ischemia: In single-center trials, DSMR was superior to dobutamine stress echocardiography for the detection of inducible wall motion abnormalities (Fig 21) in patients with suspected coronary artery disease, ${ }^{162}$ in patients with wall motion abnormalities at rest, 163 and in patients not well suited for second harmonic echocardiography. ${ }^{164}$ The superiority of DSMR is likely the result of the consistently high endocardial border visualization in the MR cine sequences that allow the detection of even subtle wall motion abnormalities. Thus, the gain in diagnostic accuracy of DSMR over dobutamine stress echocardiography is particularly high in patients with inadequate acoustic windows or limited echocardiographic image quality even when second harmonic imaging is used. The consistently high endocardial border visualization also accounts for the low interobserver variability demonstrated in a recent study of multicenter interpretation. ${ }^{165}$ In a meta-analysis of DSMR, the sensitivity for the detection of $>50 \%$ coronary diameter stenoses was $87 \%$, and the specificity was $83 \%$. 166 Landmark studies of DSMR $70,162-165,167$ are summarized in Table 4.

Identification of Myocardial Viability: Dobutamine stress MR can also be used to detect viability of dysfunctional myocardium based on the contractile response to low-dose dobutamine stimulation. Compared to delayed myocardial enhancement CMR, low-dose dobutamine CMR may be superior in predicting functional recovery after revascularization. 168 This seems particularly true in segments with nontransmural scar. As a possible explanation for this finding, delayed myocardial enhancement CMR does the exact opposite of low-dose dobutamine CMR: it images myocardial scar (ie, "nonviability"). As such, delayed myocardial enhancement CMR cannot address the functional capacity of the potentially viable myocardium surrounding the scar, and this fact may limit its ability to predict functional recovery of myocardium with nontransmural scar. For a detailed discussion of delayed myocardial enhancement CMR, we refer to the article by Bucciarelli-Ducci, et al. 57

Prognostic Value of Dobutamine Stress CMR: Several single-center studies have addressed the prognostic value of DSMR. Hundley et al ${ }^{169}$ found that the presence of inducible wall motion abnormalities during DSMR identifies patients at risk of MI and cardiac death independent of the presence of traditional risk factors for coronary artery disease. Conversely, the cardiac event rate over 2 years was low $(2 \%)$ in patients without inducible wall motion abnormalities on DSMR who had an LV ejection fraction $>40 \%$, and the cardiac event rate was $0 \%$ for patients who had an LV ejection fraction $\geq 60 \%$. 
Jahnke et al ${ }^{154}$ reported follow-up of 513 patients with known or suspected CAD for a median duration of 2.3 years. Patients with a normal DSMR had a cumulative event rate (death or MI) of 1.2, 2.6, and $3.3 \%$ in the first 3 years, whereas patients with an abnormal DSMR had a significantly higher event rate $(7.3,10.3$, and $18.8 \%$ in the first 3 years).

\section{Plaque Imaging}

Coronary atherosclerosis is a systemic, diffuse disease of the vessel wall, characterized by buildup of atherosclerotic plaque over long periods, as discussed in detail in the introductory sections of this article. All luminographic techniques including invasive selective coronary angiography, CCTA, and CMRA, tend to underestimate the true burden of atherosclerosis because subclinical early-stage atherosclerotic plaques that do not compromise the arterial lumen may go undetected. However, nonstenotic plaques are of great clinical interest, because many MIs occur because of plaque rupture in coronary segments without stenosis. ${ }^{170}$ It is well established that the risk of an acute event mediated by plaque rupture is predicated on the composition of the plaque rather than the degree of luminal narrowing. ${ }^{170}$

Atherosclerosis imaging with a variety of modalities allows visualization of subclinical disease burden in vivo, which has prognostic value. ${ }^{131}$ Due to the excellent soft-tissue contrast inherent to MRI, high-resolution imaging is considered a very promising technique for imaging the vessel wall and characterizing plaque components. ${ }^{171} \mathrm{CT}$ can visualize calcified and noncalcified plaque. CAC scoring by CT, which can be considered a form of plaque characterization and quantification and has incremental value over "traditional" multivariate risk-assessment models in selected patient groups with intermediate risk, ${ }^{106}$ has been discussed in a previous section of this issue of Current Problems in Cardiology.

Computed Tomography-The value of plaque assessment by CCTA is currently not established but is the focus of intense research. In particular, the relationship between "noncalcified" plaque (Fig 22) and histologically "soft" or biologically "unstable" plaque is not established. The implications of identifying "noncalcified" or "mixed" plaque on CCTA for patient prognosis or management are also not well established. No evidence to date supports the concept that using the presence of "noncalcified" or "mixed" plaque on CCTA as a basis of risk factor management decisions will beneficially affect patients' survival or quality of life.

Clinical in vivo CCTA can characterize the various forms of vascular remodeling that occur in response to the formation and growth of atherosclerotic plaque. For example, so-called "positive" remodeling is associated with increased risk of cardiac events. 172,173 In a recent study, Hausleiter et al ${ }^{174}$ investigated with 64-slice computed tomography (0.6-mm collimation, 330-ms gantry rotation time) the prevalence and characteristics of noncalcified coronary plaques in 161 consecutive patients with an intermediate risk of CAD. No evidence of $\mathrm{CAD}$ in the form of noncalcified or calcified plaque was found in 35 (32.9\%) patients. Coronary calcification in the absence of noncalcified plaque was found in 60 (37.3\%) patients. Noncalcified coronary plaques were detected in $48(29.8 \%)$ patients. These included 38 patients (23.6\%) in whom noncalcified plaques were found together with calcified plaque. The prevalence of noncalcified plaques as the only manifestation of CAD was low at $6.2 \%$ (10 of 161 patients). Patients with noncalcified plaques had significantly higher total cholesterol, lowdensity lipoprotein, and C-reactive protein levels, and a trend toward higher prevalence of diabetes mellitus than those who did not have noncalcified plaque. Most noncalcified plaques resulted in lumen narrowing of $<50 \%$. CCTA-based qualitative plaque characterization showed that in patients with acute coronary syndromes, culprit lesions are more likely than "stable" lesions to show positive vascular remodeling, low plaque density, and spotty calcification. ${ }^{175,176}$ 
CCTA also has been used to identify and characterize atherosclerotic plaque quantitatively. $173,177-179$ For example, when the appearance of plaques on intracoronary ultrasound is used as a standard of reference, "calcified" plaque attenuates X-rays more than "intermediate" plaque, and "intermediate" plaque attenuates X-rays more than "soft" plaque. However, the substantial overlap in HU values between the different types of plaque can make it very difficult to, with certainty, distinguish between "soft" and "intermediate" plaque. In addition, recent studies suggest that the HU value of plaques may be influenced by vessel wall enhancement during contrast injection because the vasa vasorum in the plaque are perfused with contrast as well. This finding indicates the need for standardized CCTA imaging protocols (in particular with respect to iodine content of the contrast medium and rate of contrast injection) and advanced, computerized image analysis systems to analyze the voxels representing plaque. 180 Quantification of plaque volume may eventually allow serial noninvasive examination of plaque burden to assess the efficacy of risk factor management. However, recent studies examining the accuracy and variability of plaque quantification by CCTA are divided about whether this approach is stable and reproducible enough for clinical use. ${ }^{178,181}$

Several recent studies have addressed the prognostic value of CCTA in patients with known or suspected coronary artery disease. ${ }^{182}$ In an early study in 100 patients followed for an average of 16 months, 33 cardiac events occurred in 26 patients. In patients with no evidence of plaque or stenosis on CCTA, the first-year cardiac event rate was 0 versus $30 \%$ in patients with plaques. The event rate was highest in the presence of obstructive lesions (63\%), particularly when obstructive lesions were located in the left main or left anterior descending coronary arteries $(77 \%)$. However, an increased event rate $(8 \%)$ was also observed in patients with nonobstructive $\mathrm{CAD}$ compared with patients without any plaque.

In a larger more recent single-center consecutive cohort of 1127 symptomatic patients $>45$ years followed for an average of $15.3 \pm 3.9$ months, ${ }^{183}$ CCTA identified increased risk for allcause mortality. The CCTA predictors of death included proximal left anterior descending artery stenosis, and the number of vessels with $>50$ and $>70 \%$ stenosis. ${ }^{183}$ In another study of 2538 consecutive symptomatic patients followed for an average of $78 \pm 12$ months, the burden of angiographic disease detected by CCTA provided both independent and incremental value in predicting 86 deaths independent of age, gender, conventional risk factors, and CAC. 184

Such studies provide initial data about the qualitative and quantitative assessment of plaque, and the potential prognostic value of plaque imaging with CCTA. However, their limited nature emphasizes the need for further, controlled studies before plaque assessment by CCTA can be recommended in clinical practice to assess the need for, and effect of, therapy on the prognosis of patients with cardiovascular risk factors or overt CAD.

Magnetic Resonance-Very few studies to date have addressed the ability to characterize atherosclerotic plaque in coronary arteries by CMR in vivo. The main limitation of CMR for coronary plaque characterization is its comparatively low spatial resolution. Several studies have examined the possibility of characterizing the physiology or morphology of atherosclerotic plaques in vessels with a caliber larger than that of the coronary arteries, 185 but it is not clear whether the findings from such studies readily apply to coronary artery plaques because of differences in plaque biology. The following recent studies exemplify the status of investigational in vivo coronary plaque characterization.

A combination of an ECG-gated turbo-field-echo/echo-planar sequence for bright-blood CMRA and high-resolution 3D black-blood imaging allowed characterization of atherosclerotic remodeling of the right coronary artery in 12 patients, 6 of whom had coronary artery plaque that was $<50 \%$ obstructive on invasive, selective coronary angiography. ${ }^{186}$ 
Recently, a first larger clinical trial of coronary vessel wall imaging 187 included 136 patients with longstanding type 1 diabetes without symptoms or history of cardiovascular disease, 63 (45\%) of whom had nephropathy evidenced by albuminuria. Navigator-gated CMRA and highresolution 3D black-blood imaging of the right coronary artery was performed using a standard 1.5-T scanner (Fig 23). Coronary artery stenoses were detected in $10 \%$ of the patients with nephropathy, whereas none of the patients without nephropathy had stenoses. In addition, total plaque burden expressed as mean and maximum right coronary artery vessel wall thickness was significantly greater in subjects with nephropathy.

Maintz et al 188 used inversion-recovery sequences before and after administration of a standard extracellular gadolinium compound to examine 29 plaques in nine patients. CCTA coronary angiography and invasive, selective angiography were the standards of reference. Based on their appearance on CCTA, 6 plaques were calcified, 17 mixed, and 6 noncalcified. Based on their initial appearance on CMR (bright or dark) and on the presence of enhancement (in 13 plaques [45\%]) or lack thereof after administration of gadolinium, the authors identified three types of plaque that they hypothesized represented different plaque compositions, including plaque with recent hemorrhage. In another recent study, ${ }^{189}$ the findings on delayedenhancement CMR of the coronary vessel wall using a T1-weighted 3D gradient-echo inversion recovery sequence performed 60 minutes after administration of a standard gadolinium chelate in 20 subjects ( 14 of whom had risk factors for coronary artery disease) correlated well with the presence of noncalcified plaque or CAC on MDCT.

\section{Recommendations}

Several documents endorsed by American and European professional societies of cardiologists or radiologists have reviewed and summarized the evidence on the use of CCT and CMR discussed in the previous sections of this issue of Current Problems in Cardiology. An excellent review of the role of calcification in CAD can be found in a 1996 Statement for Health Professionals from the American Heart Association. ${ }^{44}$ Some newer documents have addressed the training, competence, and equipment required for performing and interpreting CCT and CMR studies. ${ }^{190-192}$

Matthew Budoff: The newer guidelines from the American Heart Association (189, published October 2006) provide a much more up-to-date discussion of cardiac CT than the 1996 guidelines, including data on both coronary calcium scanning and CT angiography with guidelines for use.

In keeping with the fact that the evidence based for CCT and CMR is still small, no formal guidelines exist for their use, but several Consensus Statements and Scientific Statements list recommended indications for these studies. A recent "Expert Consensus Document on Coronary Artery Calcium Scoring by Computed Tomography," issued jointly by the American Heart Association and American College of Cardiology Foundation, 47 updated the evidence listed in a previous Expert Consensus Document on the same topic ${ }^{103}$ and discussed many clinical scenarios relevant to $\mathrm{CAC}$ measurement, which included the following:

1. It is reasonable to consider $\mathrm{CAC}$ measurement in asymptomatic patients with intermediate risk of CAD (10-20\% estimated 10-year risk of coronary events) because some of these patients might be reclassified to a higher risk status based on high CAC score. 106

2. Measurement of $\mathrm{CAC}$ is not recommended in asymptomatic patients with low or high risk of $\mathrm{CAD}(<10$ or $>20 \%$ estimated 10-year risk of coronary events, or established CAD). 
3. No evidence suggests that treatment intensity can be reduced in patients with intermediate risk and $\mathrm{CAC}$ score $=0$. Standard recommendations for treatment of intermediate risk patients apply.

4. Additional noninvasive or invasive testing in patients with high (> 400) CAC score is not recommended.

5. Current CAC data do not pertain to all patient groups. Data are strongest for nonHispanic Caucasian men. Caution in extrapolating data to women or ethnic minorities is recommended.

Recent scientific statements from the American Heart Association reviewed the evidence for the assessment of CAD by CCT, 193 and for noninvasive coronary artery imaging with CCTA and MRA. ${ }^{194}$ Both statements formulated indications for cardiac CT following the established practice of classifying recommendations from I to III, where class I represents "conditions for which there is evidence that a given procedure is useful and effective," and classifying the level of evidence from $\mathrm{A}$ to $\mathrm{C}$, where $\mathrm{A}$ is derived from multiple randomized trials and $\mathrm{C}$ represents consensus opinion by experts. There were no class I indications for CCTA or CMRA. The following were considered class IIa indications for CCTA:

1. Suspected CAD: CCTA is reasonable for the assessment of obstructive disease in symptomatic patients at intermediate risk for CAD (level of evidence: B). Diagnostic accuracy favors CCTA over CMRA.

2. Coronary artery anomalies: the high resolution of the data sets (permitting analysis even of small details) and the speed of image acquisition make it reasonable to use $\mathrm{CT}$ as one of the first-choice imaging modalities in the workup of known and suspected coronary anomalies (level of evidence: B in ref. ${ }^{194}$, C in ref. ${ }^{193}$ ).

Neither CCTA nor CMRA should be used to screen for CAD in patients who have no signs or symptoms suggestive of CAD. ${ }^{194}$

No possible indication for $\mathrm{CAC}$ scoring in symptomatic or asymptomatic patients was considered class I or IIa (in contradistinction to the endorsement of "CAC measurement in asymptomatic patients with intermediate risk of coronary artery disease" in the American Heart Association/American College of Cardiology Expert Consensus Statement ${ }^{47}$ discussed above). Suggestions for the interpretation of CAC scoring studies performed for class IIb indications 193 are listed in Table 5 .

A European Consensus Panel compiled clinical indications for CMR from the literature and from expert experience. ${ }^{195}$ Indications were rated from class I-III, where class I represented "clinically relevant" and "usually appropriate" indications where CMR "may be used as firstline imaging technique" because its use was "supported by substantial literature." The class I indications for cardiac MR in a strict sense (ie, excluding vascular applications) are listed in Table 6. For a complete list of level I and II indications, which include many indications related to imaging of the great or peripheral vessels, we refer the reader to the original document. ${ }^{195}$

An intersocietal working group and technical panel consisting of cardiologists and radiologists recently rated $39 \mathrm{CCT}$ and $33 \mathrm{CMR}$ indications characteristic of contemporary practice on a scale of 1-9, where scores of 7-9 indicated that the test was "generally acceptable and [was] a reasonable approach for the indication." 196 The 13 CCT and 17 CMR indications that received a median score of 7 or higher, and as such were considered appropriate, are listed in Tables 7 and 8 . Of note, among the three possible indications of "CAC scoring in asymptomatic individuals at low, moderate, or high risk for CAD," none received a median score higher than 6 (again, in contrast with the recommendation in the article by Greenland, et al. ${ }^{47}$ Among the 
three possible indications of "CCTA in asymptomatic individuals at low, moderate, or high risk for CAD," none received a median score higher than 4 .

\section{Summary}

CCT and CMR are relatively new imaging modalities that offer exciting opportunities that may exceed the ability of established imaging modalities to detect present pathology or predict patient outcomes. Studies examining the prognostic value, cost-effectiveness, and associated risks of these new imaging modalities relative to the more established ones in various clinical situations, such as the Study of myocardial Perfusion and Coronary Anatomy imaging Roles in CAD (SPARC) registry, ${ }^{197}$ are ongoing.

At the current stage of knowledge, CMR is indicated for visualizing cardiac structure and function in a variety of clinical situations, particularly to establish and evaluate congenital heart disease, and myocardial or pericardial disease. Stress CMR is useful in symptomatic patients suspected of having CAD who have suboptimal image quality or nondiagnostic findings on conventional stress tests. Delayed enhancement CMR can be considered a first-line indication for the assessment of myocardial viability.

CAC scoring may be useful in asymptomatic patients at intermediate risk (estimated 10-20\% 10 -year risk of coronary events) to refine risk assessment. CCTA is currently not indicated in asymptomatic patients but may be useful in symptomatic patients suspected of CAD who have suboptimal image quality or nondiagnostic findings on conventional stress imaging tests.

CCTA is a first-line indication to evaluate congenitally abnormal coronary arteries; however, in young patients, CMRA may be preferred to circumvent exposure to ionizing radiation.

Imaging of plaque and molecular mechanisms related to plaque rupture holds great promise for the presymptomatic detection of patients at risk for coronary events but can currently not be recommended for the evaluation or prognostication of symptomatic or asymptomatic patients. Currently, no convincing evidence suggests that modifying risk factor management based on the findings of CAC scanning or plaque imaging will affect patient outcomes.

Matthew Budoff: The St Francis Heart Study has performed a randomized trial of atorvastatin $20 \mathrm{mg}$ per day compared to placebo in patients with high coronary calcium scores. In those persons with calcium scores $>400$, treatment reduced the event rate by $42 \%(P=0.046)$. This limited study represents the only outcome study performed with coronary calcium, and no such studies have yet to be reported with plaque imaging with CTCA or MRCA.

Matthew Budoff: The field of cardiac CT and MR is in rapid evolution. Prognostic studies, serial studies, and treatment effects are being performed. The authors have carefully outlined the strengths and weaknesses of these tools and the current guidelines for their utilization.

While increasing data continue to accrue for coronary calcium assessment, including the Multi-Ethnic Study of Atherosclerosis demonstrating a 10-fold risk of cardiac events in a large ( $n=6814$ persons) population-based study, we still need to evaluate where this modality fits in with the plethora of diagnostic and prognostic tests now available in Cardiology. It appears that further risk stratification of the intermediaterisk patient will be the strongest application of this modality, given the noninvasive nature and very low radiation risk associated with this test.

CTCA and MRI of the coronaries have much less information available to assess their potential role; however, they have been greeted with great enthusiasm. Low to intermediate probability of CAD patients appears to be a good first-line use of CTCA, 
and follow-up of nondiagnostic testing with stress or stress imaging studies appears warranted. MR stress imaging and evaluation of hibernating myocardium are common clinical applications currently being used. Both modalities have advantages in coronary anomalies and heart failure. The clinician must balance the power of noninvasive visualization of the lumen or coronary plaque, with known side effects and limitations of these tools. Cost also becomes an issue, given the rising costs of health care. Certainly prudent use of these modalities until more data are available is warranted.

\section{Acknowledgments}

This work was supported in part by NIH Grant 1R01EB007986-02 ("Noninvasive Localization of Vulnerable Plaques") awarded to Dr. Birgit Kantor.

Jörg Barkhausen receives honorariums from Schering and Siemens, receives research grants from Schering and Guerbet, is a consultant for Schering and Guerbet, and is on the advisory board of Guerbet.

\section{References}

1. Libby P. Inflammation in atherosclerosis. Nature 2002;6917:868-74. [PubMed: 12490960]

2. Blackshear, JL.; Kantor, B. Pathogenesis of atherosclerosis. In: Murphy, JG., editor. Mayo Clinic Cardiology Concise Textbook. Vol. 3. London, New York: Lippincott Williamson and Wilkins; 2006. p. $699-715$.

3. Virmani R, Burke A, Farb A, et al. Pathology of the vulnerable plaque. J Am Coll Cardiol 2006;8 (suppl):C13-8. [PubMed: 16631505]

4. Jaffer F, Libby P, Weissleder R. Molecular and cellular imaging of atherosclerosis: emerging applications. J Am Coll Cardiol 2006;7:1328-38. [PubMed: 16580517]

5. Kantor B, Jorgensen S, Lund P, et al. Cryostatic micro-CT imaging of arterial wall perfusion. Scanning 2002;24:186-90. [PubMed: 12166806]

6. Stary H. Natural history and histological classification of atherosclerotic lesions: An update. Arterioscler Thromb Vasc Biol 2000;20:1177-8. [PubMed: 10807728]

7. Miller, D. Pathology of coronary artery atherosclerosis: aspects relevant to cardiac imaging. In: Gerber, T.; Kantor, B.; Williamson, E., editors. Computed Tomography for the Cardiovascular System. London, UK: Informa Healthcare; 2008.

8. Ross R. Atherosclerosis-An inflammatory disease. N Engl J Med 1999;2:115-26. [PubMed: 9887164]

9. Bjoernheden T, Evaldsson M, Wiklund O. A method for the assessment of hypoxia in the arterial wall, with potential application in vivo. Arterioscler Thromb Vasc Biol 1996;16:178-85. [PubMed: 8548420]

10. Kantor B, Moehlenkamp S. Imaging the myocardial microvasculature using fast computed tomography and three-dimensional microscopic computed tomography. Cardiol Clin 2003;21:587605. [PubMed: 14719570]

11. Moreno P, Purushothaman K, Fuster V, et al. Plaque neovascularization is increased in ruptured atherosclerotic lesions of human aorta: implications for plaque vulnerability. Circulation 2004;110:2032-8. [PubMed: 15451780]

12. Langheinrich AC, Kampschulte M, Buch T, et al. Vasa vasorum and atherosclerosis-Quid Novi? Thromb Haemost 2007;97:873-9. [PubMed: 17549287]

13. Kolodgie F, Gold H, Burke A, Virmani R. Intraplaque hemorrhage and progression of coronary atheroma. N Engl J Med 2003;349:2316-25. [PubMed: 14668457]

14. Virmani R, Kolodgie F, Burke A. Atherosclerotic plaque progression and vulnerability to rupture: angiogenesis as a source of intraplaque hemorrhage. Arterioscler Thromb Vasc Biol 2005;10:205461. [PubMed: 16037567]

15. Winter P, Caruthers SW, Wickline SA, et al. Molecular imaging by MRI. Curr Cardiol Rep 2006;1:659. [PubMed: 16507239] 
16. Winter P, Shukla H, Caruthers S, et al. Molecular imaging of human thrombus with computed tomography. Acad Radiol 2005;12(suppl 1):S9-13. [PubMed: 16106538]

17. Giachelli C, Bae N, Almeida M, et al. Osteopontin is elevated during neointima formation in rat arteries and is a novel component of human atherosclerotic plaques. J Clin Invest 1993;4:1686-96. [PubMed: 8408622]

18. Mautner G, Mautner S, Froehlich J, et al. Coronary artery calcification: assessment with electron beam CT and histomorphometric correlation. Radiology 1994;3:619-23. [PubMed: 8058924]

19. Schoenhagen P, Nissen SE, Tuzcu EM. Coronary arterial remodeling: from bench to bedside. Curr Atheroscler Rep 2003;2:150-4. [PubMed: 12573202]

20. Glagov S, Weisenberg E, Zarins C, et al. Compensatory enlargement of human atherosclerotic coronary arteries. N Engl J Med 1987;22:1371-5. [PubMed: 3574413]

21. Di Carli M, Czernin J, Hoh CK, et al. Relation among stenosis severity, myocardial blood flow, and flow reserve in patients with coronary artery disease. Circulation 1995;91:1944-51. [PubMed: 7895351]

22. Uren NG, Melin JA, De Bruyne B, et al. Relation between myocardial blood flow and the severity of coronary-artery stenosis. N Engl J Med 1994;330:1782-8. [PubMed: 8190154]

23. Gibbons RJ, Balady GJ, Bricker JT, et al. Circulation 2002;106:1883-92. [PubMed: 12356646]

24. Kumamoto M, Nakashima Y, Sueishi K. Intimal neovascularization in human coronary atherosclerosis: its origin and pathophysiological significance. Hum Pathol 1995;26:450-6. [PubMed: 7535741]

25. Fleiner M, Kummer M, Mirlacher M. Arterial neovascularization and inflammation in vulnerable patients: early and late signs of symptomatic atherosclerosis. Circulation 2004;18:2843-250. [PubMed: 15505090]

26. Langheinrich AC, Michniewicz A, Sedding DG, et al. Correlation of Vasa vasorum neovascularization and plaque progression in aortas of apolipoprotein $\mathrm{E}(-/-) /$ low-density lipoprotein (-/-) double knockout mice. Arterioscler Thromb Vasc Biol 2006;26:347-52. [PubMed: 16293797]

27. Zbinden S, Clavijo L, Kantor B, et al. Interanimal variability in preexisting collaterals is a major factor determining outcome in experimental angiogenesis trials. Am J Physiol Heart Circ Physiol 2007;4:H1891-7. [PubMed: 17189353]

28. Hyafil F, Cornily JC, Feig JE, et al. Noninvasive detection of macrophages using a nanoparticulate contrast agent for computed tomography. Nat Med 2007;13:636-41. [PubMed: 17417649]

29. Winter P, Morawski A, Caruthers S. Molecular imaging of angiogenesis in early-stage atherosclerosis with alpha(v)beta3-integrin-targeted nanoparticles. Circulation 2003;108:2270-4. [PubMed: 14557370]

30. Anderson S, Rader R, Westlin W, et al. Magnetic resonance contrast enhancement of neovasculature with alpha(v)beta(3)-targeted nanoparticles. Magn Reson Med 2000;3:433-9. [PubMed: 10975896]

31. Fayad Z, Sirol M, Nikolaou K, Choudhury R, Fuster V. Magnetic resonance imaging and computed tomography in assessment of atherosclerotic plaque. Curr Atheroscler Rep 2004;6:232-42. [PubMed: 15068749]

32. Jaffer F, Tung C, Gerszten R, Weissleder R. In vivo imaging of thrombin activity in experimental thrombi with thrombin-sensitive near-infrared molecular probe. Arterioscler Thromb Vasc Biol 2002;22:1929-35. [PubMed: 12426227]

33. Kolodgie F, Petrov A, Virmani R, et al. Targeting of apoptotic macrophages and experimental atheroma with radiolabeled annexin $\mathrm{V}$ : a technique with potential for noninvasive imaging of vulnerable plaque. Circulation 2003;108:3134-9. [PubMed: 14676140]

34. Gerber, T.; Kantor, B.; Williamson, E. Computed Tomography of the Cardiovascular System. London: Informa Healthcare; 2008.

35. Lardo, AC.; Fayad, Z.; Chronos, N., et al. Cardiovascular Magnetic Resonance: Established and Emerging Applications. London: Informa Healthcare; 2004.

36. Boyd DP, Lipton MJ. Cardiac computed tomography. Proc IEEE 1983;71:298-307.

37. Ohnesorge B, Flohr T, Becker C, et al. Cardiac imaging by means of electrocardiographically gated multisection spiral CT: initial experience. Radiology 2000;217:564-71. [PubMed: 11058661] 
38. Flohr TG, McCollough CH, Bruder H, et al. First performance evaluation of a dual-source CT (DSCT) system. Eur Radiol 2006;16:256-68. [PubMed: 16341833]

39. Achenbach S, Ropers D, Kuettner A, et al. Contrast-enhanced coronary artery visualization by dualsource computed tomography_initial experience. Eur J Radiol 2006;57:331-5. [PubMed: 16426789]

40. Gerber, T.; Walser, E. Cardiovascular computed tomography and magnetic resonance imaging. In: Murphy, JL., editor. Mayo Clinic Cardiology Concise Textbook. London, New York: Williamson, Lippincott and Wilkins; 2006. p. 185-204.

41. al Agatston AS. E. Quantification of coronary artery calcium using ultrafast computed tomography. J Am Coll Cardiol 1990;15:827-32. [PubMed: 2407762]

42. Callister TQ, Cooil B, Raya SP, et al. Coronary artery disease: improved reproducibility of calcium scoring with an electron-beam CT volumetric method. Radiology 1998;208:807-14. [PubMed: 9722864]

43. Ulzheimer S, Kalender WA. Assessment of calcium scoring performance in cardiac computed tomography. Eur Radiol 2003;13:484-97. [PubMed: 12594550]

44. Wexler L, Brundage B, Crouse J, et al. Coronary artery calcification: pathophysiology, epidemiology, imaging methods, and clinical implications. A statement for health professionals from the American Heart Association Writing Group. Circulation 1996;94:1175-92. [PubMed: 8790070]

45. Hoff JA, Chomka EV, Krainik AJ, et al. Age and gender distributions of coronary artery calcium detected by electron beam tomography in 35,246 adults. Am J Cardiol 2001;87:1335-9. [PubMed: 11397349]

46. Raggi P, Callister TQ, Cooil B, et al. Identification of patients at increased risk of first unheralded acute myocardial infarction by electron-beam computed tomography. Circulation 2000;101:850-5. [PubMed: 10694523]

47. Greenland P, Bonow RO, Brundage BH, et al. ACCF/AHA 2007 clinical expert consensus document on coronary artery calcium scoring by computed tomography in global cardiovascular risk assessment and in evaluation of patients with chest pain: a report of the American College of Cardiology Foundation Clinical Expert Consensus Task Force (ACCF/AHA Writing Committee to Update the 2000 expert consensus document on electron beam computed tomography) developed in collaboration with the Society of Atherosclerosis Imaging and Prevention and the Society of Cardiovascular Computed Tomography. J Am Coll Cardiol 2007;49:378-402. [PubMed: 17239724]

48. Gerber TC, Kuzo RS, Lane GE, et al. Image quality in a standardized algorithm for minimally invasive coronary angiography with multislice spiral computed tomography. J Comput Assist Tomogr 2003;27:62-9. [PubMed: 12544245]

49. Gerber TC, Breen JF, Kuzo RS, et al. Computed tomographic angiography of the coronary arteries: techniques and applications. Semin Ultrasound CT MR 2006;27:42-55. [PubMed: 16562571]

50. Lawler LP, Pannu HK, Fishman EK. MDCT evaluation of the coronary arteries, 2004: how we do it - data acquisition, postprocessing, display, and interpretation. AJR Am J Roentgenol 2005;184:1402-12. [PubMed: 15855087]

51. van Ooijen PM, Ho KY, Dorgelo J, et al. Coronary artery imaging with multidetector CT: visualization issues. Radiographics 2003;23:e16. [PubMed: 12907810]

52. McCollough CH, Schueler BA. Calculation of effective dose. Med Phys 2000;27:828-37. [PubMed: 10841384]

53. Thompson RC, Cullom SJ. Issues regarding radiation dosage of cardiac nuclear and radiography procedures. J Nucl Cardiol 2006;13:19-23. [PubMed: 16464713]

54. Amis ES Jr, Butler PF, Applegate KE, et al. American College of Radiology white paper on radiation dose in medicine. J Am Coll Radiol 2007;4:272-84. [PubMed: 17467608]

55. Einstein AJ, Henzlova MJ, Rajagopalan S. Estimating risk of cancer associated with radiation exposure from 64-slice computed tomography coronary angiography. JAMA 2007;298:317-23. [PubMed: 17635892]

56. (BEIR) CotBEoIR. Health Risks from Exposure to Low Levels of Ionizing Radiation: BEIR VIIPhase. Vol. 2. Washington, DC: The National Academies; 2005. 
57. Bucciarelli-Ducci C, Wu E, Lee DC, et al. Contrast-enhanced cardiac magnetic resonance in the evaluation of myocardial infarction and myocardial viability in patients with ischemic heart disease. Curr Probl Cardiol 2006;31:128-68. [PubMed: 16413381]

58. Faris OP, Shein M. Food and Drug Administration perspective: magnetic resonance imaging of pacemaker and implantable cardioverter-defibrillator patients. Circulation 2006;114:1232-3. [PubMed: 16982951]

59. Levine GN, Gomes AS, Arai AE, et al. Safety of magnetic resonance imaging in patients with cardiovascular devices: an American Heart Association scientific statement from the Committee on Diagnostic and Interventional Cardiac Catheterization, Council on Clinical Cardiology, and the Council on Cardiovascular Radiology and Intervention: endorsed by the American College of Cardiology foundation, the North American Society for Cardiac Imaging, and the Society for Cardiovascular Magnetic Resonance. Circulation 2007;116:2878-91. [PubMed: 18025533]

60. Gerber TC, Fasseas P, Lennon RJ, et al. Clinical safety of magnetic resonance imaging early after coronary artery stent placement. J Am Coll Cardiol 2003;42:1295-8. [PubMed: 14522498]

61. Kuo PH, Kanal E, Abu-Alfa AK, et al. Gadolinium-based MR contrast agents and nephrogenic systemic fibrosis. Radiology 2007;242:647-9. [PubMed: 17213364]

62. United States Food and Drug Administration (FDA). Information on gadolinium-containing contrast agents. 2008

63. Wolff SD, Schwitter J, Coulden R, et al. Myocardial first-pass perfusion magnetic resonance imaging: a multicenter dose-ranging study. Circulation 2004;110:732-7. [PubMed: 15289374]

64. Gebker R, Schwitter J, Fleck E, Nagel E. How we perform myocardial perfusion with cardiovascular magnetic resonance. J Cardiovasc Magn Reson 2007;9:539-47. [PubMed: 17365233]

65. Klocke FJ, Simonetti OP, Judd RM, et al. Limits of detection of regional differences in vasodilated flow in viable myocardium by first-pass magnetic resonance perfusion imaging. Circulation 2001;104:2412-6. [PubMed: 11705817]

66. Klem I, Heitner JF, Shah DJ, et al. Improved detection of coronary artery disease by stress perfusion cardiovascular magnetic resonance with the use of delayed enhancement infarction imaging. J Am Coll Cardiol 2006;47:1630-8. [PubMed: 16631001]

67. Cerqueira MD, Weissman NJ, Dilsizian V, et al. Standardized myocardial segmentation and nomenclature for tomographic imaging of the heart: a statement for healthcare professionals from the Cardiac Imaging Committee of the Council on Clinical Cardiology of the American Heart Association. Circulation 2002;105:539-42. [PubMed: 11815441]

68. Elkington AG, Gatehouse PD, Prasad SK, et al. Combined long- and short-axis myocardial perfusion cardiovascular magnetic resonance. J Cardiovasc Magn Reson 2004;6:811-6. [PubMed: 15646884]

69. Nagel E, Klein C, Paetsch I, et al. Magnetic resonance perfusion measurements for the noninvasive detection of coronary artery disease. Circulation 2003;108:432-7. [PubMed: 12860910]

70. Paetsch I, Jahnke C, Wahl A, et al. Comparison of dobutamine stress magnetic resonance, adenosine stress magnetic resonance, and adenosine stress magnetic resonance perfusion. Circulation 2004;110:835-42. [PubMed: 15289384]

71. Nagel E, Lorenz C, Baer F, et al. Stress cardiovascular magnetic resonance: consensus panel report. J Cardiovasc Magn Reson 2001;3:267-81. [PubMed: 11816623]

72. Cerqueira M, Verani M, Schwaiger M, et al. Safety profile of adenosine stress perfusion imaging: results from the Adenoscan Multicenter Trial Registry. J Am Coll Cardiol 1994;23:390-2. [PubMed: 8294692]

73. Wahl A, Paetsch I, Gollesch A, et al. Safety and feasibility of high-dose dobutamine-atropine stress cardiovascular magnetic resonance for diagnosis of myocardial ischaemia: experience in 1000 consecutive cases. Eur Heart J 2004;25:1230-6. [PubMed: 15246641]

74. van Geuns R, Wielopolski P, Wardeh A, et al. Volume coronary angiography using targeted scans (VCATS): a new strategy in MR coronary angiography. Int J Cardiovasc Imaging 2001;17:405-10. [PubMed: 12025954]

75. Jahnke C, Paetsch I, Achenbach S, et al. Coronary MR imaging: breath-hold capability and patterns, coronary artery rest periods, and beta-blocker use. Radiology 2006;239:71-8. [PubMed: 16493014] 
76. Stuber M, Botnar R, Danias P, et al. Double-oblique free-breathing high resolution three-dimensional coronary magnetic resonance angiography. J Am Coll Cardiol 1999;34:524-31. [PubMed: 10440168]

77. Weber O, Martin A, Higgins C. Whole-heart steady-state free precession coronary artery magnetic resonance angiography. Magn Reson Med 2003;50:1223-8. [PubMed: 14648570]

78. Brittain JH, Hu BS, Wright GA, et al. Coronary angiography with magnetization-prepared T2 contrast. Magn Reson Med 1995;33:689-96. [PubMed: 7596274]

79. Barkhausen J, Hunold P, Jochims M, et al. [Comparison of gradient-echo and steady state free precession sequences for 3D-navigator MR angiography of coronary arteries]. Rofo Fortschr Geb Rontgenstr Neuen Bildgeb Verfahr 2002;174:725-30. [PubMed: 12063602]

80. Nassenstein K, Waltering KU, Eggebrecht H, et al. [MR coronary angiography with MS-325, a blood pool contrast agent: comparison of an inversion recovery steady-state free precession with an inversion recovery fast low angle shot sequence in volunteers]. Rofo Fortschr Geb Rontgenstr Neuen Bildgeb Verfahr 2006;178:508-14. [PubMed: 16586314]

81. Dirksen MS, Lamb HJ, Kunz P, et al. Improved MR coronary angiography with use of a new rapid clearance blood pool contrast agent in pigs. Radiology 2003;227:802-8. [PubMed: 12676975]

82. Paetsch I, Jahnke C, Barkhausen J, et al. Detection of coronary stenoses with contrast enhanced, threedimensional free breathing coronary MR angiography using the gadolinium-based intravascular contrast agent gadocoletic acid (B-22956). J Cardiovasc Magn Reson 2006;8:509-16. [PubMed: 16755840]

83. Herborn CU, Barkhausen J, Paetsch I, et al. Coronary arteries: contrast-enhanced MR imaging with SH L 643 A-experience in 12 volunteers. Radiology 2003;229:217-23. [PubMed: 12944598]

84. Herborn CU, Schmidt M, Bruder O, et al. MR coronary angiography with SH L 643 A: initial experience in patients with coronary artery disease. Radiology 2004;233:567-73. [PubMed: 15358848]

85. Jahnke C, Dietrich T, Paetsch I, et al. Experimental evaluation of the detectability of submillimeter atherosclerotic lesions in ex vivo human iliac arteries with ultrahigh-field (7.0 T) magnetic resonance imaging. Int J Cardiovasc Imaging 2007;23:519-27. [PubMed: 17109199]

86. Barkhausen J, Ebert W, Heyer C, et al. Detection of atherosclerotic plaque with Gadofluorineenhanced magnetic resonance imaging. Circulation 2003;108:605-9. [PubMed: 12835227]

87. Ruehm SG, Corot C, Vogt P, et al. Magnetic resonance imaging of atherosclerotic plaque with ultrasmall superparamagnetic particles of iron oxide in hyperlipidemic rabbits. Circulation 2001;103:415-22. [PubMed: 11157694]

88. Wickline SA, Neubauer AM, Winter PM, et al. Molecular imaging and therapy of atherosclerosis with targeted nanoparticles. J Magn Reson Imaging 2007;25:667-80. [PubMed: 17347992]

89. Flacke S, Fischer S, Scott MJ, et al. Novel MRI contrast agent for molecular imaging of fibrin: implications for detecting vulnerable plaques. Circulation 2001;104:1280-5. [PubMed: 11551880]

90. Morawski AM, Winter PM, Crowder KC, et al. Targeted nanoparticles for quantitative imaging of sparse molecular epitopes with MRI. Magn Reson Med 2004;51:480-6. [PubMed: 15004788]

91. Janowitz WR, Agatston AS, Kaplan G, et al. Differences in prevalence and extent of coronary artery calcium detected by ultrafast computed tomography in asymptomatic men and women. Am J Cardiol 1993;72:247-54. [PubMed: 8342500]

92. Budoff MJ, Diamond GA, Raggi P, et al. Continuous probabilistic prediction of angiographically significant coronary artery disease using electron beam tomography. Circulation 2002;105:1791-6. [PubMed: 11956121]

93. Haberl R, Becker A, Leber A, et al. Correlation of coronary calcification and angiographically documented stenoses in patients with suspected coronary artery disease: results of 1,764 patients. J Am Coll Cardiol 2001;37:451-7. [PubMed: 11216962]

94. Rumberger JA, Simons DB, Fitzpatrick LA, et al. Coronary artery calcium area by electron-beam computed tomography and coronary atherosclerotic plaque area. A histopathologic correlative study. Circulation 1995;92:2157-62. [PubMed: 7554196]

95. Simons DB, Schwartz RS, Edwards WD, et al. Noninvasive definition of anatomic coronary artery disease by ultrafast computed tomographic scanning: a quantitative pathologic comparison study. $\mathrm{J}$ Am Coll Cardiol 1992;20:1118-26. [PubMed: 1401612] 
96. Berman DS, Wong ND, Gransar H, et al. Relationship between stress-induced myocardial ischemia and atherosclerosis measured by coronary calcium tomography. J Am Coll Cardiol 2004;44:923-30. [PubMed: 15312881]

97. Knez A, Becker A, Leber A, et al. Relation of coronary calcium scores by electron beam tomography to obstructive disease in 2,115 symptomatic patients. Am J Cardiol 2004;93:1150-2. [PubMed: 15110209]

98. Laudon DA, Vukov LF, Breen JF, et al. Use of electron-beam computed tomography in the evaluation of chest pain patients in the emergency department. Ann Emerg Med 1999;33:15-21. [PubMed: 9867882]

99. Lee RT, Grodzinsky AJ, Frank EH, et al. Structure-dependent dynamic mechanical behavior of fibrous caps from human atherosclerotic plaques. Circulation 1991;83:1764-70. [PubMed: 2022029]

100. Cheng GC, Loree HM, Kamm RD, et al. Distribution of circumferential stress in ruptured and stable atherosclerotic lesions. A structural analysis with histopatho-logical correlation. Circulation 1993;87:1179-87. [PubMed: 8462145]

101. Fitzgerald PJ, Ports TA, Yock PG. Contribution of localized calcium deposits to dissection after angioplasty. An observational study using intravascular ultrasound. Circulation 1992;86:64-70. [PubMed: 1617791]

102. Schmermund A, Erbel R. Unstable coronary plaque and its relation to coronary calcium. Circulation 2001;104:1682-7. [PubMed: 11581149]

103. O'Rourke RA, Brundage BH, Froelicher VF, et al. American College of Cardiology/American Heart Association expert consensus document on electron-beam computed tomography for the diagnosis and prognosis of coronary artery disease. Circulation 2000;102:126-40. [PubMed: 10880426]

104. Pletcher MJ, Tice JA, Pignone M, et al. Using the coronary artery calcium score to predict coronary heart disease events: a systematic review and metal-analysis. Arch Intern Med 2004;164:1285-92. [PubMed: 15226161]

105. Arad Y, Goodman KJ, Roth M, et al. Coronary calcification, coronary disease risk factors, C-reactive protein, and atherosclerotic cardiovascular disease events: the St. Francis heart study. J Am Coll Cardiol 2005;46:158-65. [PubMed: 15992651]

106. Greenland P, LaBree L, Azen SP, et al. Coronary artery calcium score combined with Framingham score for risk prediction in asymptomatic individuals. JAMA 2004;291:210-5. [PubMed: 14722147]

107. Kondos GT, Hoff JA, Sevrukov A, et al. Electron-beam tomography coronary artery calcium and cardiac events: a 37-month follow-up of 5635 initially asymptomatic low-to intermediate-risk adults. Circulation 2003;107:2571-6. [PubMed: 12743005]

108. LaMonte MJ, FitzGerald SJ, Church TS, et al. Coronary artery calcium score and coronary heart disease events in a large cohort of asymptomatic men and women. Am J Epidemiol 2005;162:4219. [PubMed: 16076829]

109. Taylor AJ, Bindeman J, Feuerstein I, et al. Coronary calcium independently predicts incident premature coronary heart disease over measured cardiovascular risk factors: mean three-year outcomes in the prospective Army Coronary Calcium (PACC) project. J Am Coll Cardiol 2005;46:807-14. [PubMed: 16139129]

110. Vliegenthart R, Oudkerk M, Hofman A, et al. Coronary calcification improves cardiovascular risk prediction in the elderly. Circulation 2005;112:572-7. [PubMed: 16009800]

111. Yoon HC, Emerick AM, Hill JA, et al. Calcium begets calcium: Progression of coronary artery calcification in asymptomatic subjects. Radiology 2002;224:236-41. [PubMed: 12091689]

112. Budoff MJ, Lane KL, Bakhsheshi H, et al. Rates of progression of coronary calcium by electron beam tomography. Am J Cardiol 2000;86:8-11. [PubMed: 10867084]

113. Maher JE, Bielak LF, Raz JA, et al. Progression of coronary artery calcification: a pilot study. Mayo Clin Proc 1999;74:347-55. [PubMed: 10221462]

114. Raggi P, Callister TQ, Shaw LJ. Progression of coronary artery calcium and risk of first myocardial infarction in patients receiving cholesterol-lowering therapy. Arterioscler Thromb Vasc Biol 2004;24:1272-7. [PubMed: 15059806] 
115. Raggi P, Cooil B, Ratti C, et al. Progression of coronary artery calcium and occurrence of myocardial infarction in patients with and without diabetes mellitus. Hypertension 2005;46:238-43. [PubMed: 15851627]

116. Raggi P, Cooil B, Shaw LJ, et al. Progression of coronary calcium on serial electron beam tomographic scanning is greater in patients with future myocardial infarction. Am J Cardiol 2003;92:827-9. [PubMed: 14516885]

117. Raggi P, Davidson M, Callister TQ, et al. Aggressive versus moderate lipid-lowering therapy in hypercholesterolemic postmenopausal women: beyond endorsed lipid lowering with EBT scanning (BELLES). Circulation 2005;112:563-71. [PubMed: 16009795]

118. Schmermund A, Achenbach S, Budde T, et al. Effect of intensive versus standard lipid-lowering treatment with atorvastatin on the progression of calcified coronary atherosclerosis over 12 months: a multicenter, randomized, double-blind trial. Circulation 2006;113:427-37. [PubMed: 16415377]

119. Schoenhagen P, Halliburton SS, Stillman AE, et al. Noninvasive imaging of coronary arteries: current and future role of multi-detector row CT. Radiology 2004;232:7-17. [PubMed: 15220490]

120. Schoepf UJ, Becker CR, Ohnesorge BM, et al. Of coronary artery disease. Radiology 2004;232:1837. [PubMed: 15220491]

121. Gould KL, Kirkeeide RL, Buchi M. Coronary flow reserve as a physiologic measure of stenosis severity. J Am Coll Cardiol 1990;15:459-74. [PubMed: 2137151]

122. Pijls NH, De Bruyne B, Peels K, et al. Measurement of fractional flow reserve to assess the functional severity of coronary-artery stenoses. N Engl J Med 1996;334:1703-8. [PubMed: 8637515]

123. White CW, Wright CB, Doty DB, et al. Does visual interpretation of the coronary arteriogram predict the physiologic importance of a coronary stenosis? N Engl J Med 1984;310:819-24. [PubMed: 6700670]

124. Hamon M, Biondi-Zoccai GG, Malagutti P, et al. Diagnostic performance of multislice spiral computed tomography of coronary arteries as compared with conventional invasive coronary angiography: a metal-analysis. J Am Coll Cardiol 2006;48:1896-910. [PubMed: 17084268]

125. Garcia MJ, Lessick J, Hoffmann MH. Accuracy of 16-row multidetector computed tomography for the assessment of coronary artery stenosis. JAMA 2006;296:403-11. [PubMed: 16868298]

126. Hoffmann U, Moselewski F, Cury RC, et al. Predictive value of 16-slice multidetector spiral computed tomography to detect significant obstructive coronary artery disease in patients at high risk for coronary artery disease: patient-versus segment-based analysis. Circulation 2004;110:2638-43. [PubMed: 15492297]

127. Raff GL, Gallagher MJ, O’Neill WW, et al. Diagnostic accuracy of noninvasive coronary angiography using 64-slice spiral computed tomography. J Am Coll Cardiol 2005;46:552-7. [PubMed: 16053973]

128. Stein PD, Yaekoub AY, Matta F, et al. 64-Slice CT for diagnosis of coronary artery disease: a systematic review. Am J Med 2008;121:715-25. [PubMed: 18691486]

129. Dewey M, Dubel HP, Schink T, et al. Head-to-head comparison of multislice computed tomography and exercise electrocardiography for diagnosis of coronary artery disease. Eur Heart J. 2006(Epub ahead of print)

130. Mollet NR, Cademartiri F, Van Mieghem C, et al. Adjunctive value of CT coronary angiography in the diagnostic work-up of patients with typical angina pectoris. Eur Heart J 2007;28:1872-8. [PubMed: 17350972]

131. Sankatsing RR, de Groot E, Jukema JW, et al. Surrogate markers for atherosclerotic disease. Curr Opin Lipidol 2005;16:434-41. [PubMed: 15990593]

132. Ghostine S, Caussin C, Habis M, et al. Non-invasive diagnosis of ischaemic heart failure using 64slice computed tomography. Eur Heart J 2008;29(17):2133-40.

133. Scheffel H, Leschka S, Plass A, et al. Accuracy of 64-slice computed tomography for the preoperative detection of coronary artery disease in patients with chronic aortic regurgitation. Am J Cardiol 2007;100:701-6. [PubMed: 17697832]

134. Ghostine S, Caussin C, Daoud B, et al. Non-invasive detection of coronary artery disease in patients with left bundle branch block using 64-slice computed tomography. J Am Coll Cardiol 2006;48:1929-34. [PubMed: 17112979] 
135. Stillman AE, Oudkerk M, Ackerman M, et al. Use of multidetector computed tomography for the assessment of acute chest pain: a consensus statement of the North American Society of Cardiac Imaging and the European Society of Cardiac Radiology. Int J Cardiovasc Imaging 2007;23:41527. [PubMed: 17492364]

136. Goldstein JA, Gallagher MJ, O’Neill WW, et al. A randomized controlled trial of multi-slice coronary computed tomography for evaluation of acute chest pain. J Am Coll Cardiol 2007;49:86371. [PubMed: 17320744]

137. Vrachliotis TG, Bis KG, Haidary A, et al. Atypical chest pain: Coronary, aortic, and pulmonary vasculature enhancement at biphasic single-injection 64-section CT angiography. Radiology 2007;243:368-76. [PubMed: 17400761]

138. Kim WY, Danias PG, Stuber M, et al. Coronary magnetic resonance angiography for the detection of coronary stenoses. N Engl J Med 2001;345:1863-9. [PubMed: 11756576]

139. Sommer T, Hofer U, Hackenbroch M, et al. [Submillimeter 3D coronary MR angiography with realtime navigator correction in 107 patients with suspected coronary artery disease]. Rofo Fortschr Geb Rontgenstr Neuen Bildgeb Verfahr 2002;174:459-66. [PubMed: 11960409]

140. Schuijf JD, Bax JJ, Shaw LJ, et al. Meta-analysis of comparative diagnostic performance of magnetic resonance imaging and multislice computed tomography for noninvasive coronary angiography. Am Heart J 2006;151:404-11. [PubMed: 16442907]

141. Kefer J, Coche E, Legros G, et al. Head-to-head comparison of three-dimensional navigator-gated magnetic resonance imaging and 16-slice computed tomography to detect coronary artery stenosis in patients. J Am Coll Cardiol 2005;46:92-100. [PubMed: 15992641]

142. Dewey M, Teige F, Schnapauff D, et al. Noninvasive detection of coronary artery stenoses with multislice computed tomography or magnetic resonance imaging. Ann Intern Med 2006;145:40715. [PubMed: 16983128]

143. Dewey M, Teige F, Schnapauff D, et al. Combination of free-breathing and breathhold steady-state free precession magnetic resonance angiography for detection of coronary artery stenoses. J Magn Reson Imaging 2006;23:674-81. [PubMed: 16568418]

144. Nagel, E.; Pettigrew, RI. Myocardial ischemia in conditions other than atheromatous coronary artery disease. In: Anagnostopoulos, CD.; Bax, JJ.; Nihouannopoulos, P., et al., editors. Noninvasive Imaging of Myocardial Ischemia. London: Springer Verlag; 2006. p. 227-86.

145. Eagle KA, Guyton RA, Davidoff R, et al. ACC/AHA 2004 guideline update for coronary artery bypass graft surgery: summary article: a report of the American College of Cardiology/American Heart Association Task Force on Practice guidelines (Committee to Update the 1999 guidelines for coronary artery bypass graft surgery). Circulation 2004;110:1168-76. [PubMed: 15339866]

146. Smith SC Jr, Feldman TE, Hirshfeld JW Jr, et al. ACC/AHA/SCAI 2005 guideline update for percutaneous coronary intervention-summary article: a report of the American College of Cardiology/American Heart Association Task Force on Practice guidelines (ACC/AHA/SCAI Writing Committee to Update the 2001 guidelines for percutaneous coronary intervention). Circulation 2006;113:156-75. [PubMed: 16391169]

147. Giang TH, Nanz D, Coulden R, et al. Detection of coronary artery disease by magnetic resonance myocardial perfusion imaging with various contrast medium doses: first European multi-centre experience. Eur Heart J 2004;25:1657-65. [PubMed: 15351166]

148. Lee DC, Simonetti OP, Harris KR, et al. Magnetic resonance versus radionuclide pharmacological stress perfusion imaging for flow-limiting stenoses of varying severity. Circulation 2004;110:5865. [PubMed: 15210596]

149. Al-Saadi N, Nagel E, Gross M, et al. Noninvasive detection of myocardial ischemia from perfusion reserve based on cardiovascular magnetic resonance. Circulation 2000;101:1379-83. [PubMed: 10736280]

150. Ibrahim T, Nekolla SG, Schreiber K, et al. Assessment of coronary flow reserve: comparison between contrast-enhanced magnetic resonance imaging and positron emission tomography. J Am Coll Cardiol 2002;39:864-70. [PubMed: 11869854]

151. Plein S, Radjenovic A, Ridgway JP, et al. Coronary artery disease: myocardial perfusion MR imaging with sensitivity encoding versus conventional angiography. Radiology 2005;235:423-30. [PubMed: 15858084] 
152. Schwitter J, Nanz D, Kneifel S, et al. Assessment of myocardial perfusion in coronary artery disease by magnetic resonance: a comparison with positron emission tomography and coronary angiography. Circulation 2001;103:2230-5. [PubMed: 11342469]

153. Ingkanisorn WP, Kwong RY, Bohme NS, et al. Prognosis of negative adenosine stress magnetic resonance in patients presenting to an emergency department with chest pain. J Am Coll Cardiol 2006;47:1427-32. [PubMed: 16580532]

154. Jahnke C, Nagel E, Gebker R, et al. Prognostic value of cardiac magnetic resonance stress tests. Adenosine stress perfusion and dobutamine stress wall motion imaging. Circulation 2007;115:e457-9. [PubMed: 17502581]

155. Georgiou D, Wolfkiel C, Brundage BH. Ultrafast computed tomography for the physiological evaluation of myocardial perfusion. Am J Card Imaging 1994;8:151-8. [PubMed: 8032187]

156. George RT, Silva C, Cordeiro MA, et al. Multidetector computed tomography myocardial perfusion imaging during adenosine stress. J Am Coll Cardiol 2006;48:153-60. [PubMed: 16814661]

157. Mahnken AH, Gunther RW, Krombach G. [Contrast-enhanced MR and MSCT for the assessment of myocardial viability]. Rofo Fortschr Geb Rontgenstr Neuen Bildgeb Verfahr 2006;178:771-80. [PubMed: 16862503]

158. Nikolaou K, Sanz J, Poon M, et al. Assessment of myocardial perfusion and viability from routine contrast-enhanced 16-detector-row computed tomography of the heart: preliminary results. Eur Radiol 2005;15:864-71. [PubMed: 15776243]

159. Nieman K, Cury RC, Ferencik M, et al. Differentiation of recent and chronic myocardial infarction by cardiac computed tomography. Am J Cardiol 2006;98:303-8. [PubMed: 16860013]

160. Lardo AC, Cordeiro MA, Silva C, et al. Contrast-enhanced multidetector computed tomography viability imaging after myocardial infarction: characterization of myocyte death, microvascular obstruction, and chronic scar. Circulation 2006;113:394-404. [PubMed: 16432071]

161. Gerber BL, Belge B, Legros GJ, et al. Characterization of acute and chronic myocardial infarcts by multidetector computed tomography: comparison with contrast-enhanced magnetic resonance. Circulation 2006;113:823-33. [PubMed: 16461822]

162. Nagel E, Lehmkuhl HB, Bocksch W, et al. Noninvasive diagnosis of ischemia-induced wall motion abnormalities with the use of high-dose dobutamine stress MRI: comparison with dobutamine stress echocardiography. Circulation 1999;99:763-70. [PubMed: 9989961]

163. Wahl A, Paetsch I, Roethemeyer S, et al. High-dose dobutamine-atropine stress cardiovascular MR imaging after coronary revascularization in patients with wall motion abnormalities at rest.

Radiology 2004;233:210-6. [PubMed: 15304662]

164. Hundley WG, Hamilton CA, Thomas MS, et al. Utility of fast cine magnetic resonance imaging and display for the detection of myocardial ischemia in patients not well suited for second harmonic stress echocardiography. Circulation 1999;100:1697-702. [PubMed: 10525488]

165. Paetsch I, Jahnke C, Ferrari VA, et al. Determination of interobserver variability for identifying inducible left ventricular wall motion abnormalities during dobutamine stress magnetic resonance imaging. Eur Heart J 2006;27:1459-64. [PubMed: 16613929]

166. Mandapaka S, Hundley WG. Dobutamine cardiovascular magnetic resonance: a review. J Magn Reson Imaging 2006;24:499-512. [PubMed: 16892202]

167. van Rugge FP, van der Wall EE, de Roos A, et al. Dobutamine stress magnetic resonance imaging for detection of coronary artery disease. J Am Coll Cardiol 1993;22:431-9. [PubMed: 8335812]

168. Wellnhofer E, Olariu A, Klein C, et al. Magnetic resonance low-dose dobutamine test is superior to SCAR quantification for the prediction of functional recovery. Circulation 2004;109:2172-4. [PubMed: 15117834]

169. Hundley WG, Morgan TM, Neagle CM, et al. Magnetic resonance imaging determination of cardiac prognosis. Circulation 2002;106:2328-33. [PubMed: 12403662]

170. Falk E, Shah PK, Fuster V. Coronary plaque disruption. Circulation 1995;92:657-71. [PubMed: 7634481]

171. Fayad ZA, Fuster V. Clinical imaging of the high-risk or vulnerable atherosclerotic plaque. Circul Res 2001;89:305-16. 
172. Achenbach S, Ropers D, Hoffmann U, et al. Assessment of coronary remodeling in stenotic and nonstenotic coronary atherosclerotic lesions by multidetector spiral computed tomography. J Am Coll Cardiol 2004;43:842-7. [PubMed: 14998627]

173. Schoenhagen P, Tuzcu EM, Stillman AE, et al. Non-invasive assessment of plaque morphology and remodeling in mildly stenotic coronary segments: Comparison of 16-slice computed tomography and intravascular ultrasound. Coron Artery Dis 2003;14:459-62. [PubMed: 12966267]

174. Hausleiter J, Meyer T, Hadamitzky M, et al. Prevalence of noncalcified coronary plaques by 64slice computed tomography in patients with an intermediate risk for significant coronary artery disease. J Am Coll Cardiol 2006;48:312-8. [PubMed: 16843181]

175. Hoffmann U, Moselewski F, Nieman K, et al. Noninvasive assessment of plaque morphology and composition in culprit and stable lesions in acute coronary syndrome and stable lesions in stable angina by multidetector computed tomography. J Am Coll Cardiol 2006;47:1655-62. [PubMed: 16631006]

176. Motoyama S, Kondo T, Sarai M, et al. Multislice computed tomographic characteristics of coronary lesions in acute coronary syndromes. J Am Coll Cardiol 2007;50:319-26. [PubMed: 17659199]

177. Schroeder S, Kopp AF, Baumbach A, et al. Noninvasive detection and evaluation of atherosclerotic coronary plaques with multislice computed tomography. J Am Coll Cardiol 2001;37:1430-5. [PubMed: 11300457]

178. Achenbach S, Moselewski F, Ropers D, et al. Detection of calcified and noncal-cified coronary atherosclerotic plaque by contrast-enhanced, submillimeter multi-detector spiral computed tomography: a segment-based comparison with intravascular ultrasound. Circulation 2004;109:147. [PubMed: 14691045]

179. Leber AW, Knez A, Becker A, et al. Accuracy of multidetector spiral computed tomography in identifying and differentiating the composition of coronary atherosclerotic plaques: a comparative study with intracoronary ultrasound. J Am Coll Cardiol 2004;43:1241-7. [PubMed: 15063437]

180. Halliburton SS, Schoenhagen P, Nair A, et al. Contrast enhancement of coronary atherosclerotic plaque: a high-resolution, multidetector-row computed tomography study of pressure-perfused, human ex vivo coronary arteries. Coron Artery Dis 2006;17:553-60. [PubMed: 16905968]

181. Leber AW, Becker A, Knez A, et al. Accuracy of 64-slice computed tomography to classify and quantify plaque volumes in the proximal coronary system: a comparative study using intravascular ultrasound. J Am Coll Cardiol 2006;47:672-7. [PubMed: 16458154]

182. Pundziute G, Schuijf JD, Jukema JW, et al. Prognostic value of multislice computed tomography coronary angiography in patients with known or suspected coronary artery disease. J Am Coll Cardiol 2007;49:62-70. [PubMed: 17207724]

183. Min JK, Shaw LJ, Devereux RB, et al. Prognostic value of multidetector coronary computed tomographic angiography for prediction of all-cause mortality. J Am Coll Cardiol 2007;50:116170. [PubMed: 17868808]

184. Ostrom MP, Gopal A, Ahmadi N, et al. Mortality incidence and the severity of coronary atherosclerosis assessed by computed tomography angiography. J Am Coll Cardiol. 2009(in press)

185. Kerwin WS, O'Brien KD, Ferguson MS, et al. Inflammation in carotid atherosclerotic plaque: a dynamic contrast-enhanced MR imaging study. Radiology 2006;241:459-68. [PubMed: 16966482]

186. Kim WY, Stuber M, Bornert P, et al. Three-dimensional black-blood cardiac magnetic resonance coronary vessel wall imaging detects positive arterial remodeling in patients with nonsignificant coronary artery disease. Circulation 2002;106:296-9. [PubMed: 12119242]

187. Kim WY, Astrup AS, Stuber M, et al. Subclinical coronary and aortic atherosclerosis detected by magnetic resonance imaging in Type 1 diabetes with and without diabetic nephropathy. Circulation 2007;115:228-35. [PubMed: 17190865]

188. Maintz D, Ozgun M, Hoffmeier A, et al. Selective coronary artery plaque visualization and differentiation by contrast-enhanced inversion prepared MRI. Eur Heart J 2006;27:1732-6. [PubMed: 16787955]

189. Yeon SB, Sabir A, Clouse M, et al. Delayed-enhancement cardiovascular magnetic resonance coronary artery wall imaging: comparison with multislice computed tomography and quantitative coronary angiography. J Am Coll Cardiol 2007;50:441-7. [PubMed: 17662397] 
190. Budoff MJ, Cohen MC, Garcia MJ, et al. ACCF/AHA clinical competence statement on cardiac imaging with computed tomography and magnetic resonance. Circulation 2005;112:598-617. [PubMed: 16046290]

191. Woodard PK, Bluemke DA, Cascade PN, et al. ACR practice guideline for the performance and interpretation of cardiac magnetic resonance imaging (MRI). J Am Coll Radiol 2006;3:665-76. [PubMed: 17412147]

192. Budoff MJ, Achenbach S, Berman DS, et al. Task force 13: training in advanced cardiovascular imaging (computed tomography) endorsed by the American Society of Nuclear Cardiology, Society of Atherosclerosis Imaging and Prevention, Society for Cardiovascular Angiography and Interventions, and Society of Cardiovascular Computed Tomography. J Am Coll Cardiol 2008;51:409-14. [PubMed: 18206762]

193. Budoff MJ, Achenbach S, Blumenthal RS, et al. Assessment of coronary artery disease by cardiac computed tomography: a scientific statement from the American Heart Association Committee on Cardiovascular Imaging and Intervention, Council on cardiovascular radiology and intervention, and Committee on Cardiac Imaging, Council on Clinical Cardiology. Circulation 2006;114:176191. [PubMed: 17015792]

194. Bluemke DA, Achenbach S, Budoff M, et al. Noninvasive coronary artery imaging: magnetic resonance angiography and multidetector computed tomography angiography: a scientific statement from the American Heart Association committee on cardiovascular imaging and intervention of the council on cardiovascular radiology and intervention, and the councils on clinical cardiology and cardiovascular disease in the young. Circulation 2008;118:586-606. [PubMed: 18586979]

195. Pennell DJ, Sechtem UP, Higgins CB, et al. Clinical indications for cardiovascular magnetic resonance (CMR): Consensus Panel report. Eur Heart J 2004;25:1940-65. [PubMed: 15522474]

196. Hendel RC, Patel MR, Kramer CM, et al. ACCF/ACR/SCCT/SCMR/ASNC/NASCI/SCAI/SIR 2006 appropriateness criteria for cardiac computed tomography and cardiac magnetic resonance imaging: a report of the American College of Cardiology Foundation Quality Strategic Directions Committee Appropriateness Criteria Working Group, American College of Radiology, Society of Cardiovascular Computed Tomography, Society for Cardiovascular Magnetic Resonance, American Society of Nuclear Cardiology, North American Society for Cardiac Imaging, Society for Cardiovascular Angiography and Interventions, and Society of Interventional Radiology. J Am Coll Cardiol 2006;48:1475-97. [PubMed: 17010819]

197. Study of perfusion and anatomy's role in CAD. [Accessed on Feb 5, 2009]. Available at http://clinicaltrials.gov/ct/show/NCT00321399?order=1 


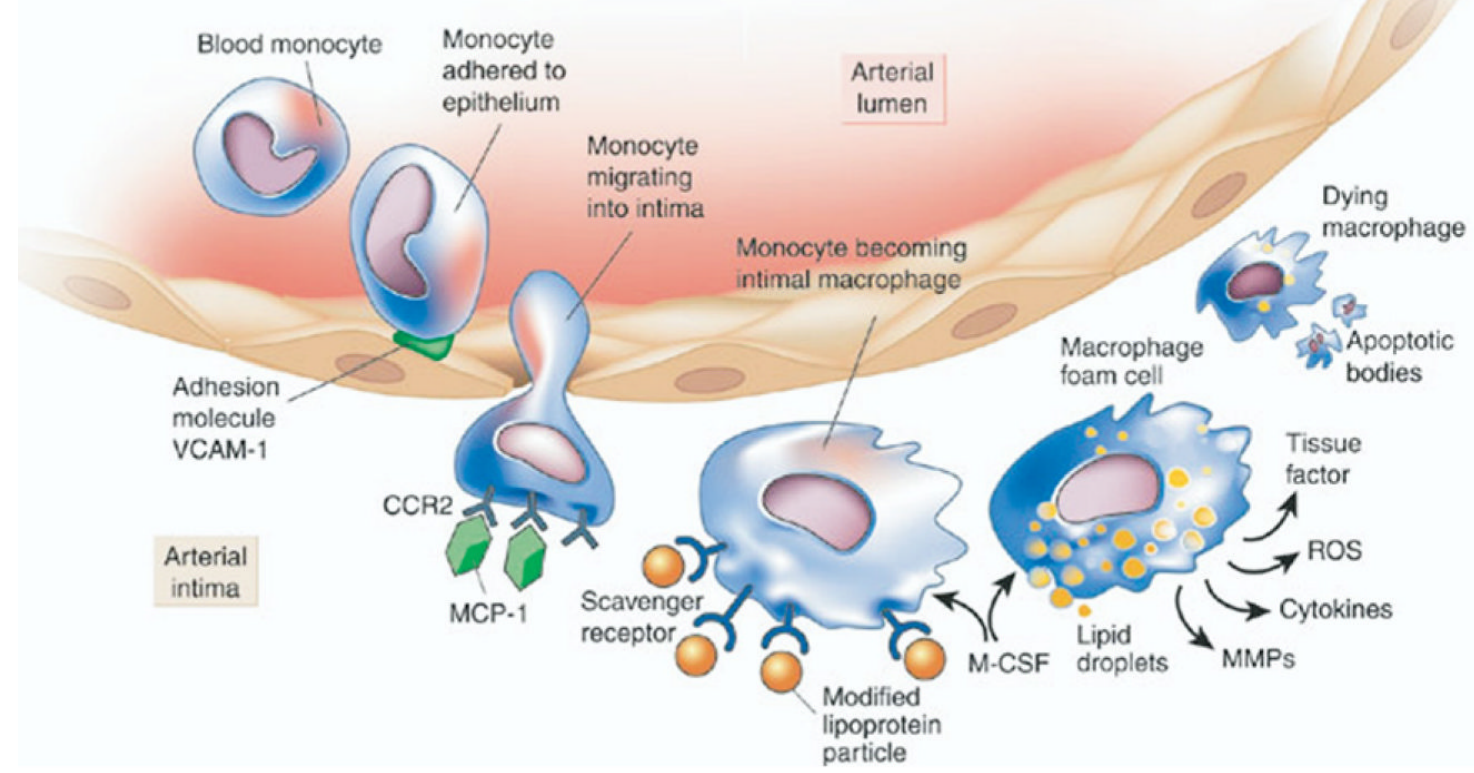

FIG 1.

The biology of atherosclerosis initiation, progression, and complications. This figure illustrates some of the important targets for molecular imaging that are discussed in the text. CCR2, chemokine (CC motif) receptor 2; $M C P$, monocyte chemoattractant protein; $M$ - $C S F$, monocyte colony-stimulating factor; $M M P$, matrix metalloproteinase; $R O S$, reactive oxygen species; $V C A M$, vascular cell adhesion molecule. (Reprinted with permission from Libby P. Inflammation in atherosclerosis. Nature 2002;6917:868-74.) 


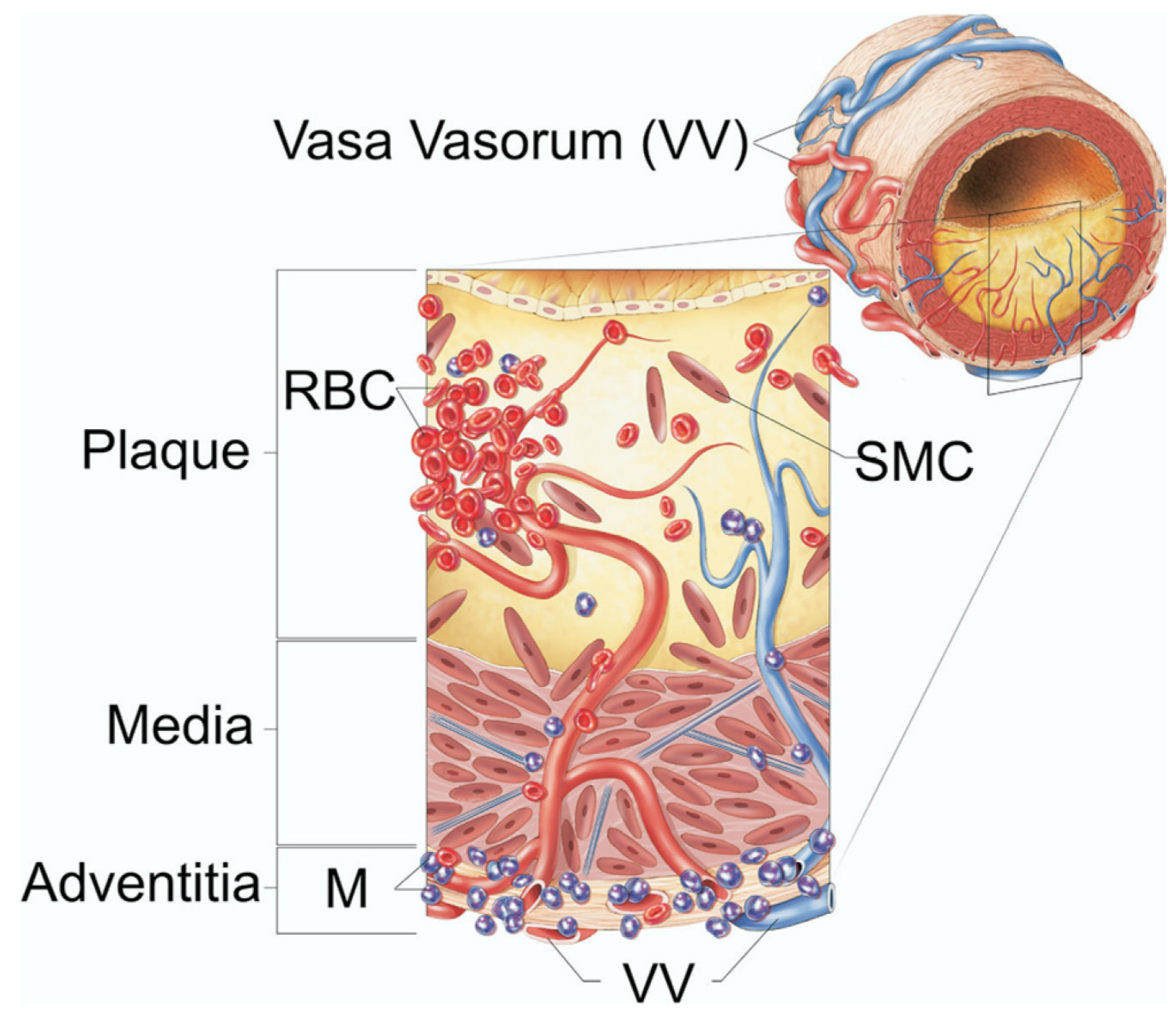

FIG 2.

Schematic illustration summarizing the role of vasa vasorum in intraplaque hemorrhage of advanced atherosclerotic lesions. Red blood cells or macrophages can be detected after their dissolution by the iron deposits that remain in their place. $R B C$, red blood cells; $M$, monocytes; $S M C$, smooth muscle cells; $V V$, vasa vasorum. (Reprinted with permission from Langheinrich AC, Kampschulte M, Buch T, et al. Vasa vasorum and atherosclerosis-Quid Novi? Thromb Haemost 2007;97:873-9.) 

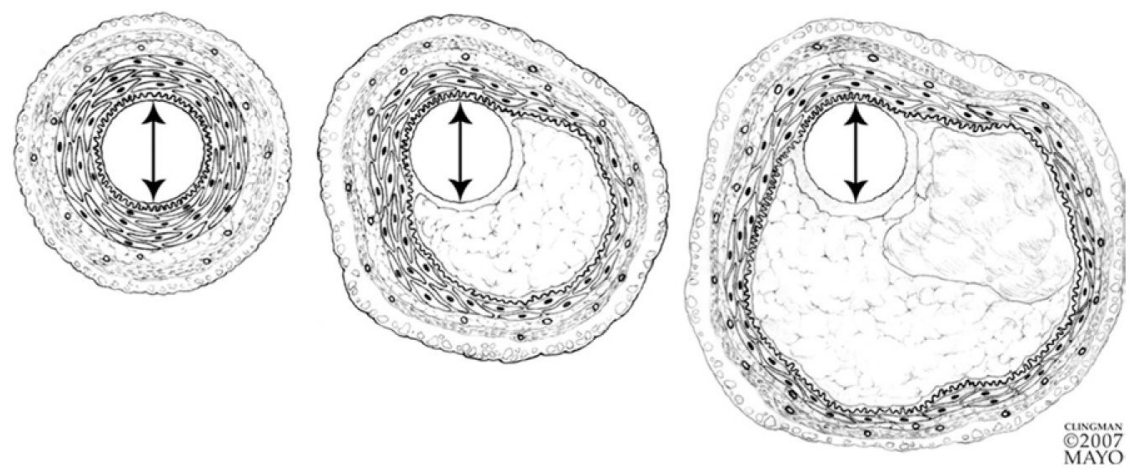

FIG 3.

As a plaque progresses in size (left to right), compensatory changes occur in the vessel wall that result in dilatation of vessel cross section and preservation of the original luminal diameter. The media underlying a plaque undergoes atrophy and the smooth muscle of the plaque-free wall hypertrophies. (Reprinted with permission from Miller D. Pathology of coronary artery atherosclerosis: aspects relevant to cardiac imaging. In: Gerber T, Kantor B, Williamson E, editors. Computed Tomography for the Cardiovascular System. London, UK: Informa Healthcare, 2008.) 

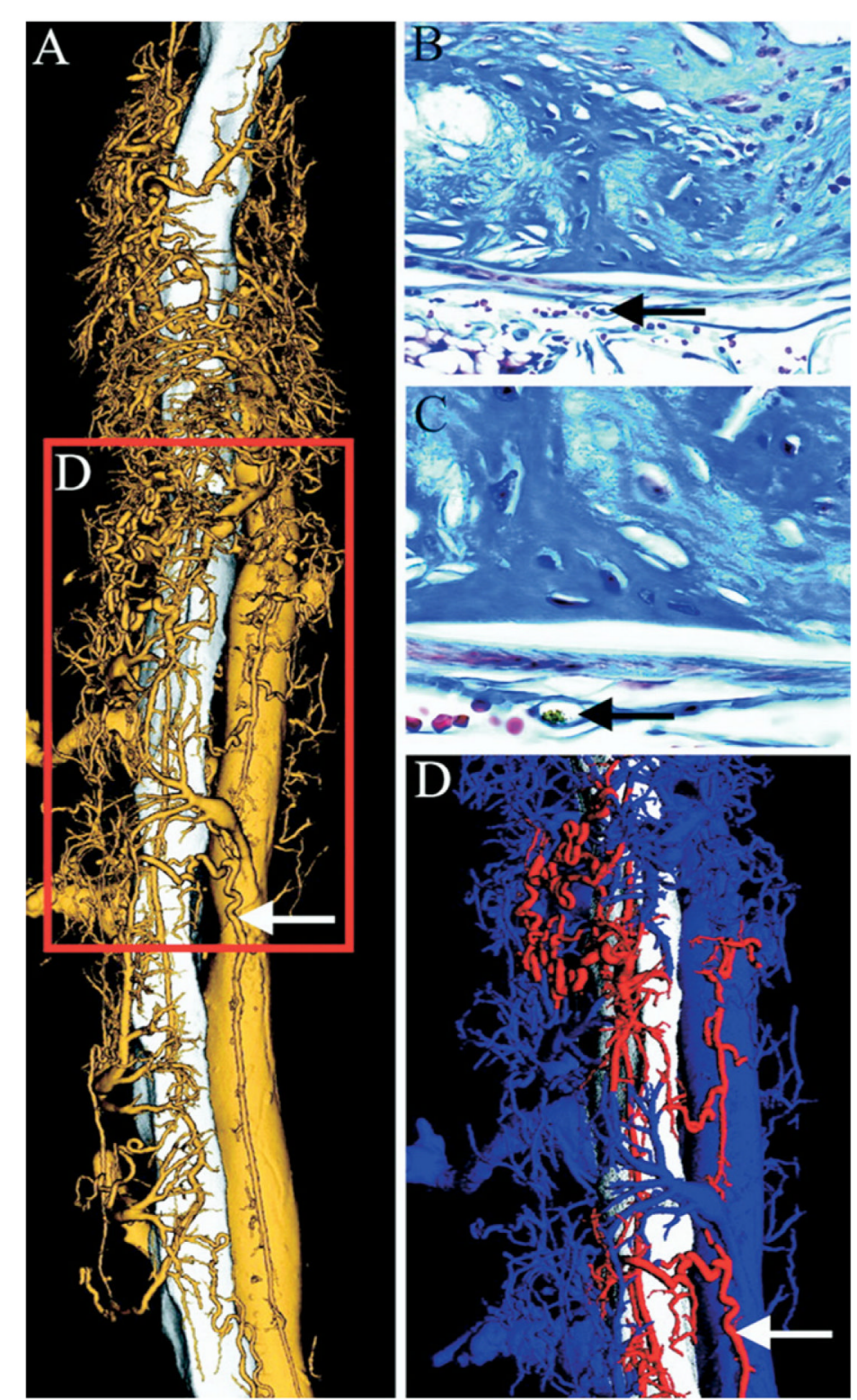

FIG 4.

Volume-rendered high-resolution microscopic $\mathrm{CT}$ image of the descending aorta, vasa vasorum (VV), and the inferior vena cava. (A) Demonstration of the $3 \mathrm{D}$ relationship of the VV (gold) to the aorta (white) (5- $\mu \mathrm{m}$ cubic voxels). (B and C) Corresponding histologic crosssections of atherosclerotic lesions in the inferior vena cava with VV (black arrow) (Masson's trichrome stain, original magnification $\times 40[B]$ and $\times 100[C])$. (D) Arterial (red) and venous (blue) VV differentiated by false color. (Reprinted with permission from Langheinrich AC, Michniewicz A, Sedding DG, et al. Correlation of Vasa vasorum neovascularization and plaque progression in aortas of apolipoprotein $\mathrm{E}(-/-) /$ low-density lipoprotein $(-/-)$ double knockout mice. Arterioscler Thromb Vasc Biol 2006;26:347-52.) 


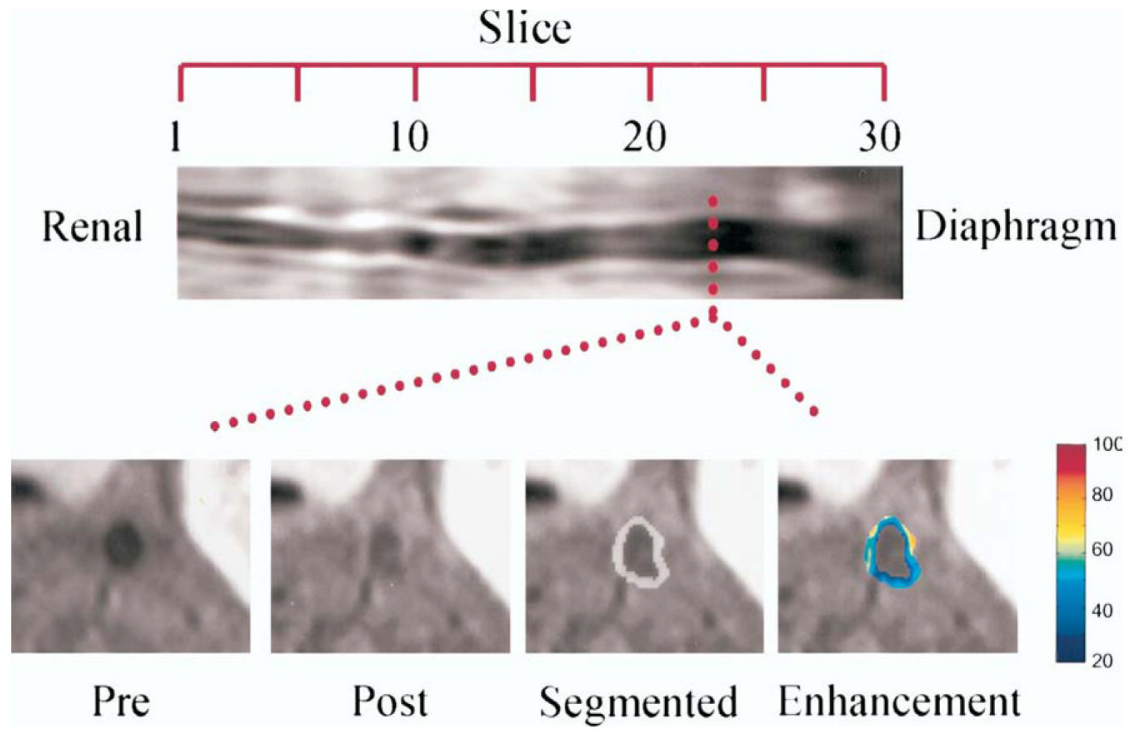

FIG 5.

In vivo spin-echo image reformatted to display the aorta of one cholesterol-fed rabbit in long axis from the renal arteries to the diaphragm (top) and at single transverse level (bottom) before (pre) and after (post) treatment; images are displayed after semiautomated segmentation (segmented, grayish ring), and with color-coded signal enhancement (enhancement) above baseline (in percent). (Reprinted with permission from Winter P, Morawski A, Caruthers S. Molecular imaging of angiogenesis in early-stage atherosclerosis with alpha(v)beta3-integrintargeted nanoparticles. Circulation 2003;108:2270-4.) 


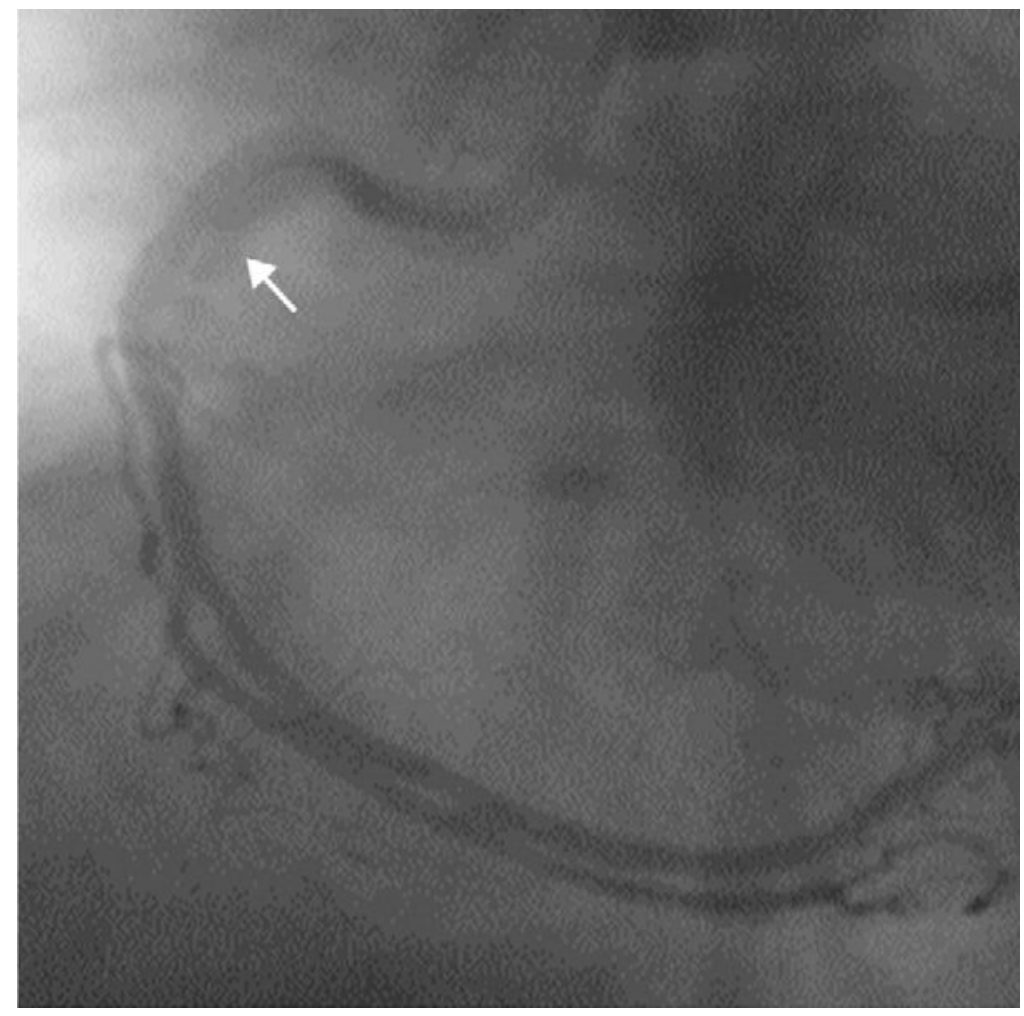

FIG 6.

Coronary imaging is complicated by the constant rapid cardiac and coronary motion. This is demonstrated in this figure from an invasive, selective coronary angiogram. Despite a temporal resolution of approximately $20 \mathrm{~ms}$, motion artifact (arrow, indicates blurring) can still be seen in some still images (typically during systole). 

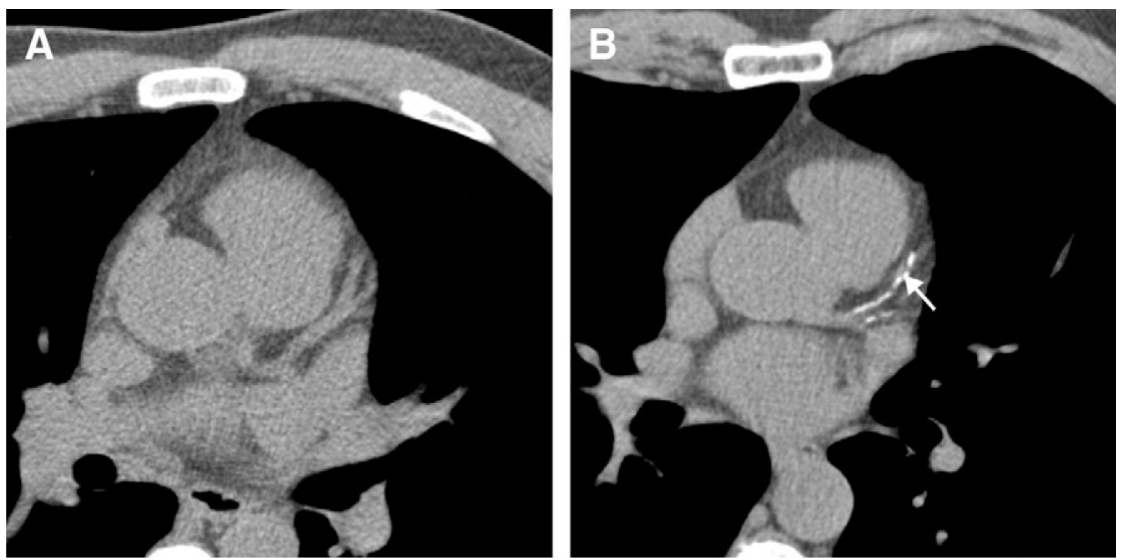

FIG 7.

MDCT images of the heart without contrast enhancement. (A) without coronary artery calcifications. (B) Patient with calcification of the left anterior descending artery (arrow) and the intermediate branch. (Reprinted with permission from Gerber T, Walser E. Cardiovascular computed tomography and magnetic resonance imaging. In: Murphy JL, editor. Mayo Clinic Cardiology Concise Textbook. London, New York: Williamson, Lippincott and Wilkins, 2006. p. 185-204.) 

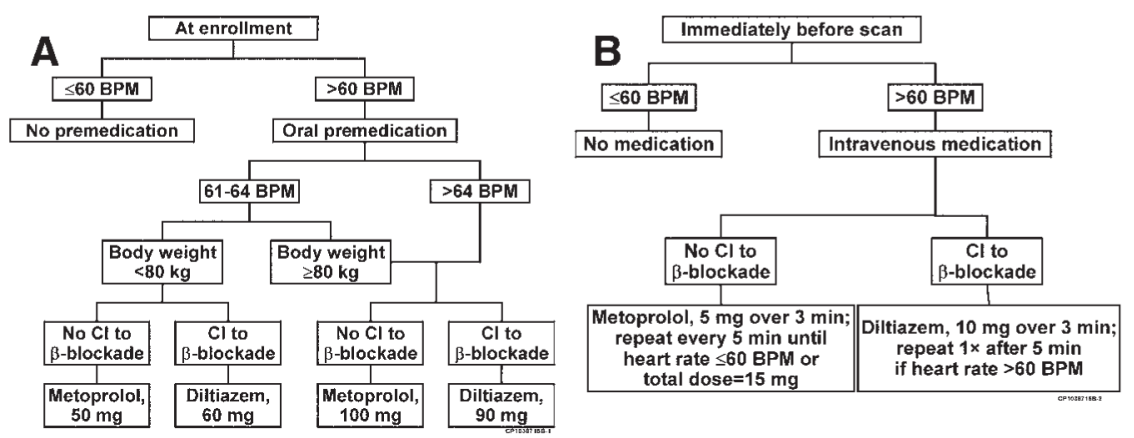

FIG 8.

The algorithm used by Mayo Clinic physicians in Florida for pharmacologic heart rate control. (A) At enrollment. (B) Immediately before scan. BPM, beats per minute; $C I$, contraindication. (Reprinted with permission from ref. Gerber TC, Kuzo RS, Lane GE, et al. Image quality in a standardized algorithm for minimally invasive coronary angiography with multislice spiral computed tomography. J Comput Assist Tomogr 2003;27:62-9.) 


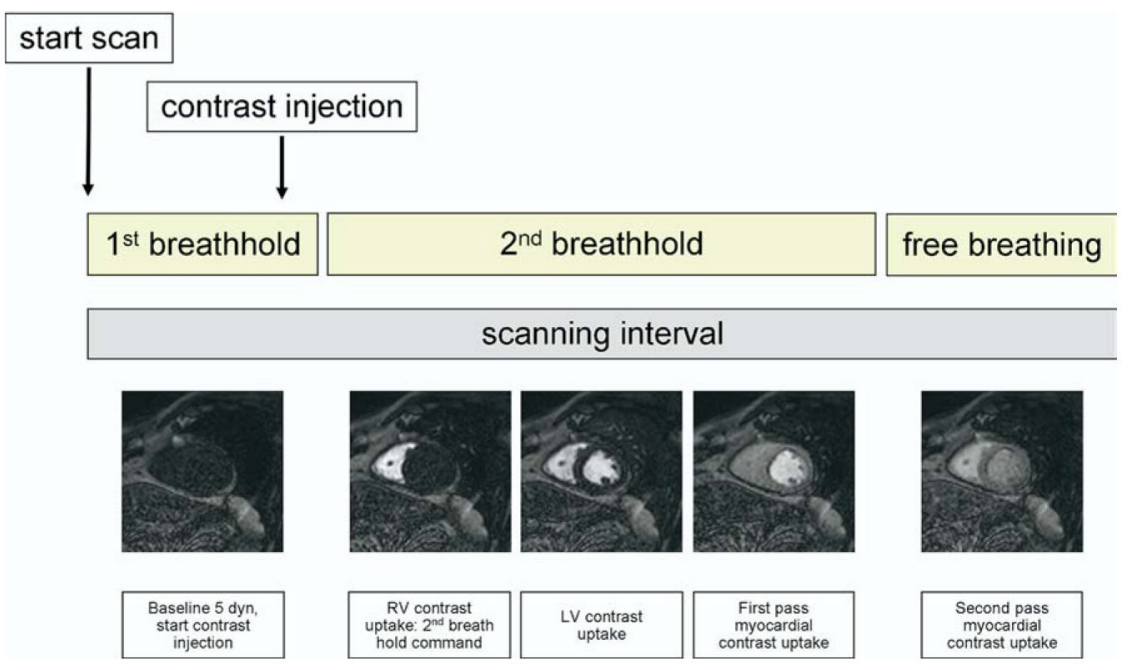

FIG 9.

Technique of breath-holding during a first pass myocardial perfusion MRI study. MRI, magnetic resonance imaging. (Reprinted with permission from Gebker R, Schwitter J, Fleck E, Nagel E. How we perform myocardial perfusion with cardiovascular magnetic resonance. J Cardiovasc Magn Reson 2007;9:539-47.) 

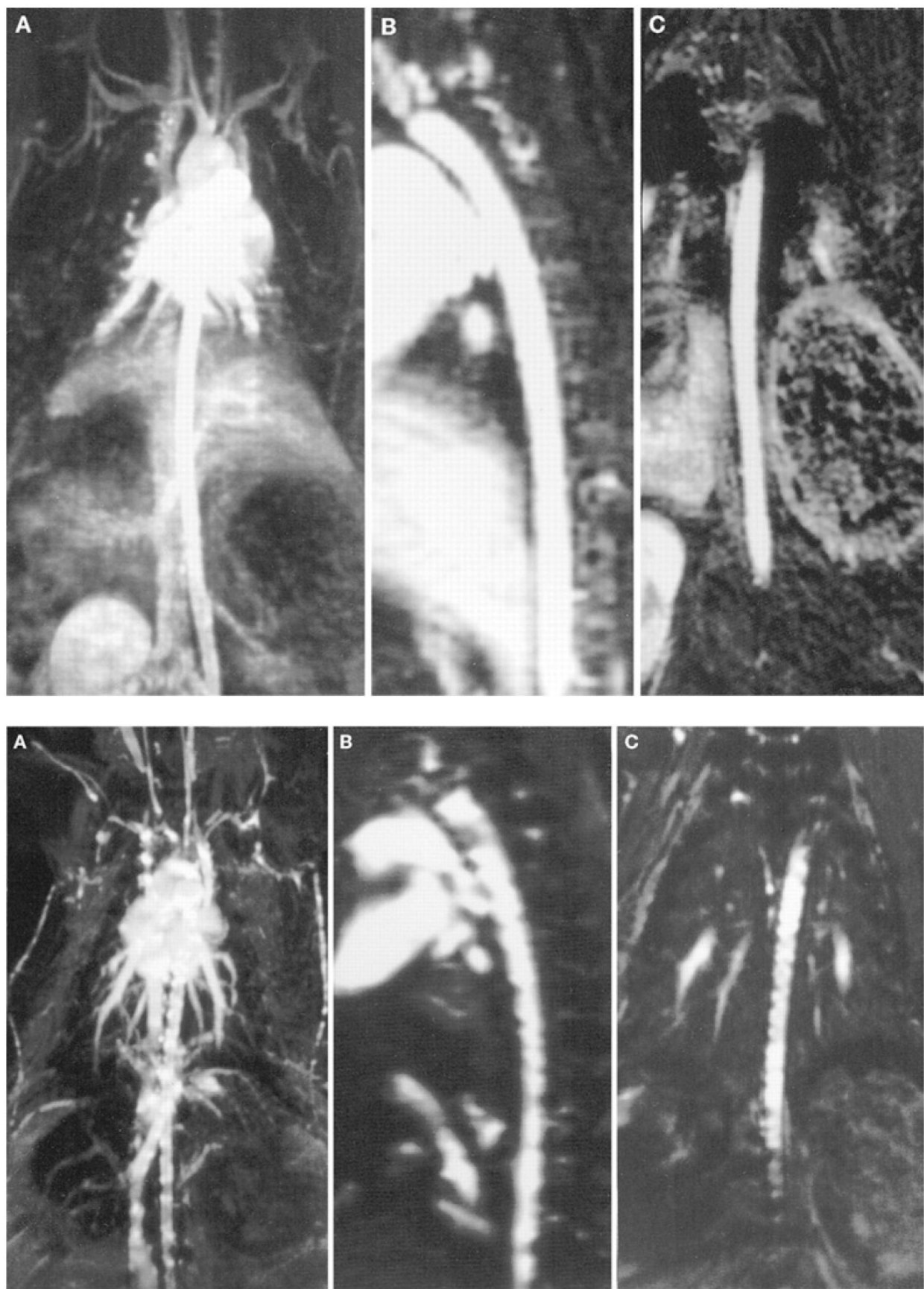

FIG 10.

Coronal maximum intensity projection (upper row, A), and images reformatted in sagittal oblique (upper row, B) and coronal oblique (upper row, C) projection from a contrast-enhanced 3D magnetic resonance angiography data set collected after intravenous administration of $\mathrm{Gd}-$ dota. The aorta of a 7-month-old hyperlipidemic rabbit is displayed. The aortic wall is smooth, without evidence of luminal narrowing. Lower panel, A-C, images of contrast-enhanced 3D magnetic resonance angiography data sets of same hyperlipidemic rabbit displayed in the same fashion as in upper panels, obtained 5 days after intravenous injection of ultrasmall superparamagnetic particles of iron oxide. Susceptibility effects, seen as irregularities within the vessel wall, represent iron uptake in macrophages that are embedded in the plaque. 3D, three- 
dimensional. (Reprinted with permission from Ruehm SG, Corot C, Vogt P, et al. Magnetic resonance imaging of atherosclerotic plaque with ultrasmall superparamagnetic particles of iron oxide in hyperlipidemic rabbits. Circulation 2001;103:415-22.) 


\section{$\alpha-$ Fibrin NP}

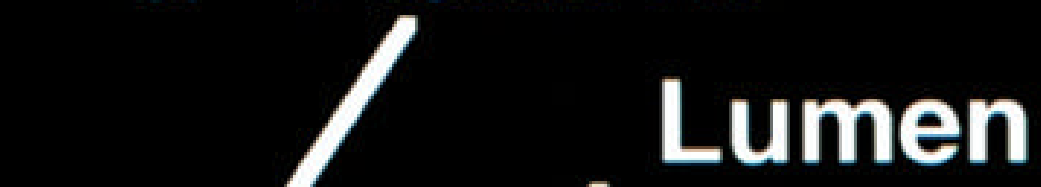

\section{Plasma}
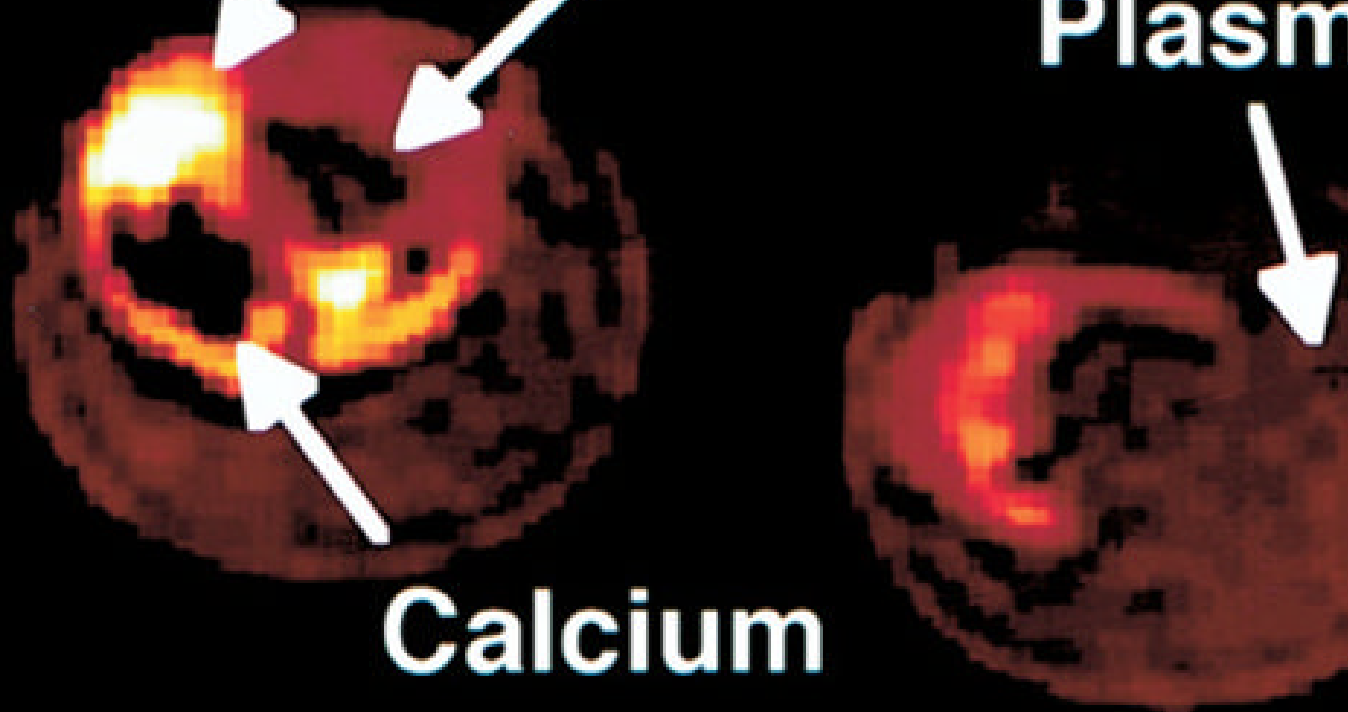

\section{Targeted}

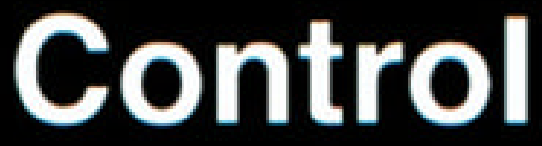

FIG 11.

Color-enhanced MRIs of fibrin-targeted and control carotid endarterectomy specimens.

Contrast enhancement (white) of a small fibrin deposit on ruptured plaque is seen, along with a calcium deposit (black). 3D, fat-suppressed, T1-weighted fast gradient echo. $N P$, nanoparticle. (Reprinted with permission from Flacke S, Fischer S, Scott MJ, et al. Novel MRI contrast agent for molecular imaging of fibrin: implications for detecting vulnerable plaques. Circulation 2001;104:1280-5.) 

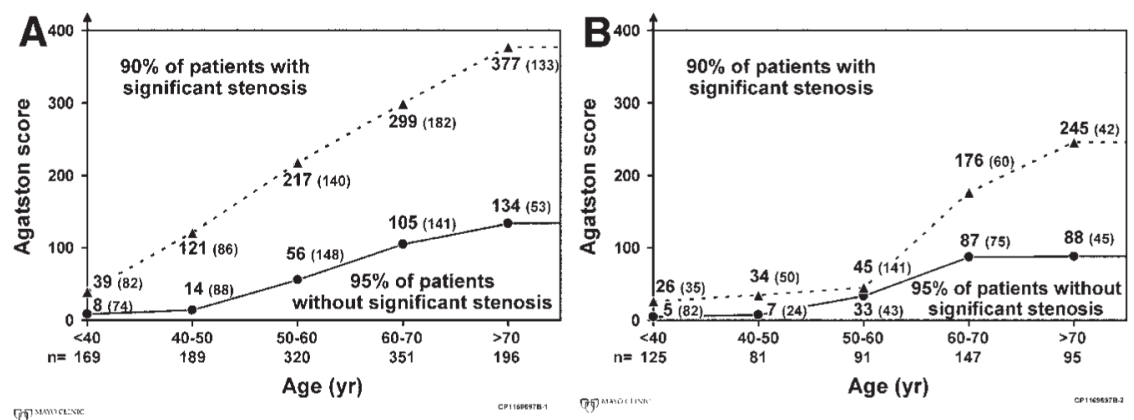

FIG 12.

Age- and gender-specific thresholds of the Agatston score signifying high or low likelihood of flow-limiting coronary artery stenoses in 1764 symptomatic patients who underwent coronary artery calcium scoring before invasive coronary angiography. (A) Data for 1225 men. (B) Data for 539 women. Ninety-five percent of patients with Agatston scores below the lower threshold values (solid line) had no significant coronary artery stenoses. The numbers in parentheses next to the threshold values represent the numbers of patients in this group (lower gray area). Ninety percent of patients with Agatston scores above the upper threshold values (broken line) had significant coronary artery stenoses. The numbers in parentheses next to the threshold values represent the numbers of patients in this group (upper gray area). At intermediate Agatston scores (white area), the probability of significant coronary artery disease, was indeterminate. Eight percent of men and 7\% of women fell into this group. (Reprinted with permission from Haberl R, Becker A, Leber A, et al. Correlation of coronary calcification and angiographically documented stenoses in patients with suspected coronary artery disease: results of 1,764 patients. J Am Coll Cardiol 2001;37:451-7.) 


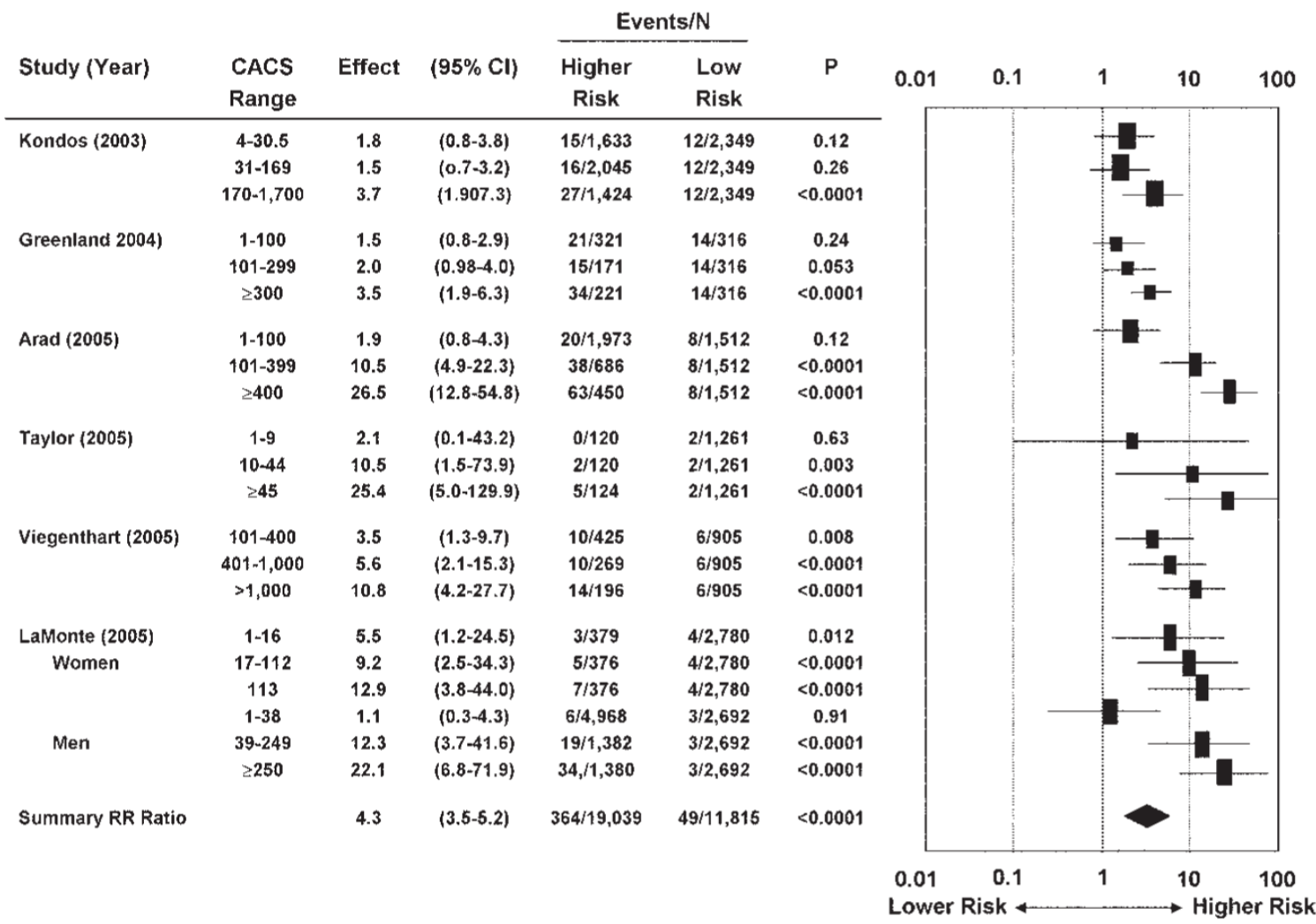

FIG 13.

Meta-analysis of the prognostic value of coronary artery calcium. Relative risk ratios (95\% confidence intervals) in six published reports. $C A C S$, coronary artery calcium score. (Reprinted with permission from Greenland P, Bonow RO, Brundage BH, et al. ACCF/AHA 2007 clinical expert consensus document on coronary artery calcium scoring by computed tomography in global cardiovascular risk assessment and in evaluation of patients with chest pain: a report of the American College of Cardiology Foundation Clinical Expert Consensus Task Force (ACCF/AHA Writing Committee to Update the 2000 expert consensus document on electron beam computed tomography) developed in collaboration with the Society of Atherosclerosis Imaging and Prevention and the Society of Cardiovascular Computed Tomography. J Am Coll Cardiol 2007;49:378-402.) 

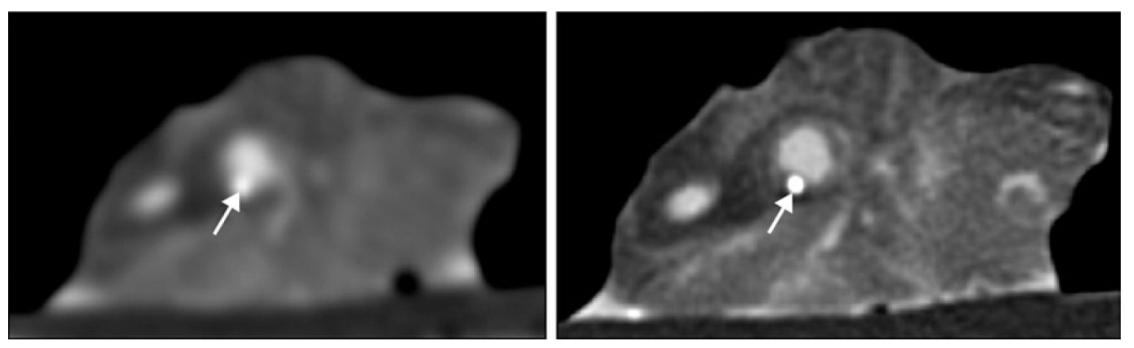

FIG 14.

Lesion calcifications cause characteristic "calcium-blooming" artifacts (arrows) on CT images. The influence of CT technology is demonstrated in this figure, which shows a small vessel wall calcification imaged ex vivo with low (left) and high (right) resolution. The calciumblooming is reduced in the high-resolution scan on the right. 

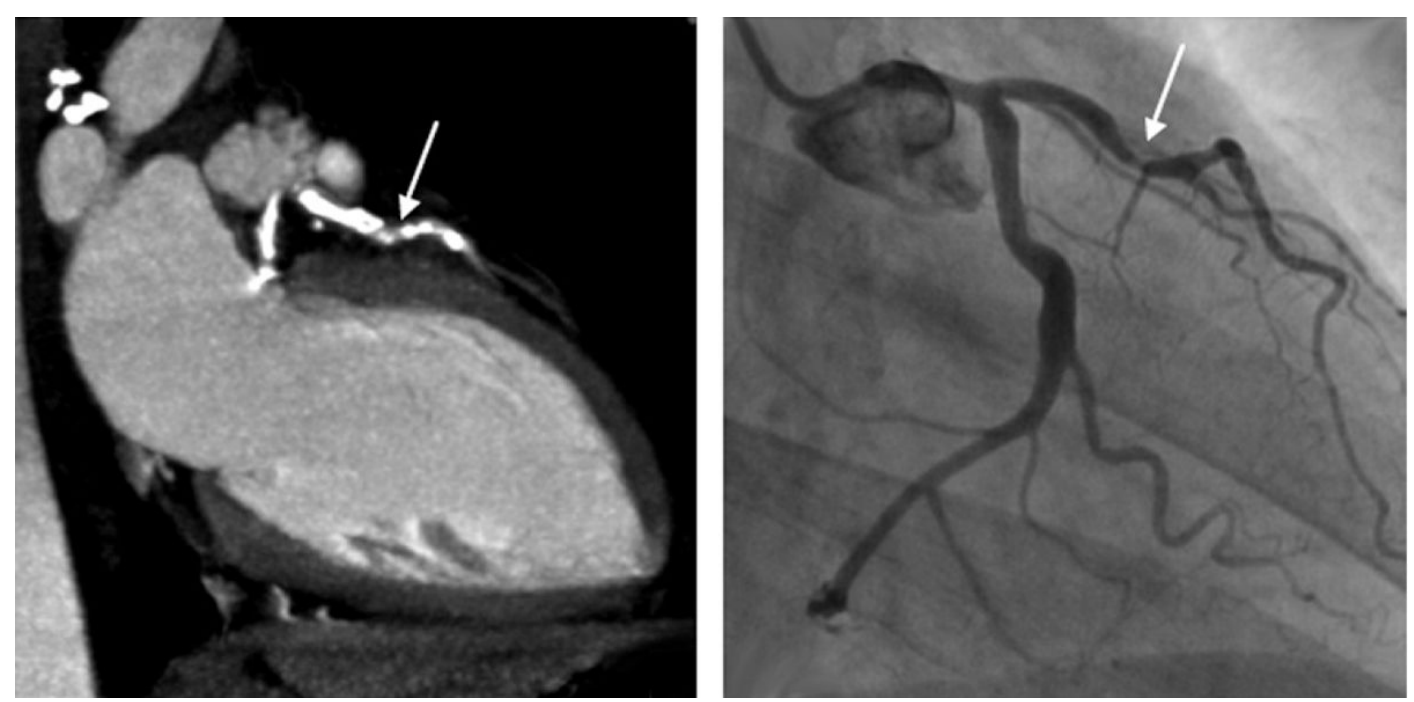

FIG 15.

In typical patients with known coronary artery disease, dense lesion calcification with associated blooming artifact can lead to overestimation of lesion (arrows) severity or can preclude assessment of densely calcified segments altogether. 


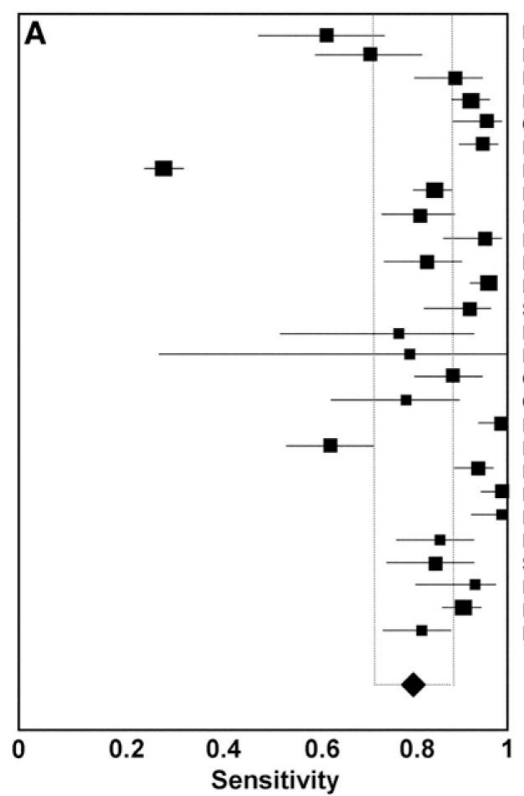

offman (2004 16-slice) Kuettner (2004 16-slice) Martuscelli (2004 16-slice) Mollet (2004 16-slice) Cademartiri (2005 16-slice) Hoffman (2005 16-slice) Kaiser (2005 16-slice) Kuettner (2005 I 16-slice) Kuettner (2005 II 16-slice)
Mollet (2005 16-slice) Morgan-H. (2005 16-slice) Probst (2005 16-slice) Schuijf (2005 16-slice) Reant (2006 16-slice) Nikolaou (2006 16-slice) Garcia (2006 16-slice) Cordeiro (2005 32-slice) $\operatorname{Lim}(2006$ 40-slice) Leber (2005 64-slice) Leschka (2005 64-slice) Mollet (2005 64-slice) Pugliese (2005 64-slice) Raff (2005 64-slice) Schuijf (2006 64-slice) Ropers (2006 64-slice) Ehara (2006 64-slice) Nikolaou (2006 64-slice)

Pooled Sensitivity $=0.81(0.72$ to 0.89$)$

Chi-square $=844.38 ; \mathrm{df}=26(p=0.0000)$

Inconsistency (I-square) $=96.9 \%$

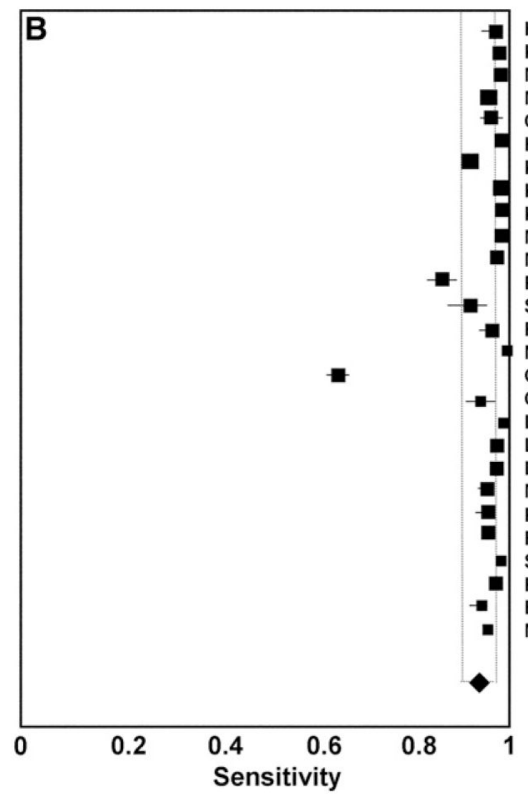

Hoffman (2004 16-slice) Kuettner (2004 16-slice) Martuscelli (2004 16-slice) Mollet (2004 16-slice) Cademartiri (2005 16-slice) Hoffman (2005 16-slice) Kuettner (2005 | 16-slice) Kuettner (2005 || 16-slice) Mollet (2005 16-slice) Morgan-H. (2005 16-slice)

Probst (2005 16-slice)

Schuijf (2005 16-slice)

Reant (2006 16-slice)

Nikolaou (2006 16-slice) Garcia (2006 16-slice)

Cordeiro (2005 32-slice)

Lim (2006 40-slice)

Leber (2005 64-slice)

Leschka (2005 64-slice)

Mollet (2005 64-slice)

Pugliese (2005 64-slice)

Raff (2005 64-slice)

Schuijf (2006 64-slice)

Ropers (2006 64-slice)

Ehara (2006 64-slice)

Nikolaou (2006 64-slice)

Sensitivity

$0.96 \quad(0.94-0.98)$

$0.97 \quad(0.95-0.98)$

$\begin{array}{ll}0.98 & (0.97-0.99) \\ 0.95 & (0.94-0.96)\end{array}$

$0.96 \quad(0.93=0.98)$

$0.98 \quad(0.97-0.99)$

$0.91 \quad(0.90-0.93)$

$0.98 \quad(0.97-0.98)$

$0.98 \quad(0.97-0.98)$

$\begin{array}{ll}0.98 & (0.97-0.99) \\ 0.98 & (0.97-0.99)\end{array}$

$0.98 \quad(0.97-0.99)$

$0.86 \quad(0.82-0.89)$

$0.91 \quad(0.87=0.94)$

$0.96 \quad(0.93-0.97)$

$0.99 \quad(0.98-1.00)$

$0.65 \quad(0.62-0.67)$

$0.94 \quad(0.90-0.97)$

$\begin{array}{ll}0.98 & (0.97-0.99) \\ 0.97 & (0.96-0.98)\end{array}$

$0.97 \quad(0.96-0.98)$

$0.95 \quad(0.93-0.97)$

$0.96 \quad(0.93-0.97)$

$0.96 \quad(0.93-0.97)$
$0.95 \quad(0.93-0.97)$

$0.95 \quad(0.93-0.97)$

$0.94 \quad(0.96-0.98)$

$0.94 \quad(0.92-0.96)$

Pooled Specificity $=0.93(0.90$ to 0.97$)$

Chi-square $=1566.24 ; \mathrm{df}=26(p=0.0000)$

Inconsistency (I-square) $=98.3 \%$

FIG 16.

(A) Plot and table of per-segment sensitivity and (B) specificity of CCTA compared with invasive, selective coronary angiography. $C I$, confidence interval; $d . f$., degrees of freedom. (Reprinted with permission from Hamon M, Biondi-Zoccai GG, Malagutti P, et al. Diagnostic performance of multislice spiral computed tomography of coronary arteries as compared with conventional invasive coronary angiography: a metal-analysis. J Am Coll Cardiol 2006;48:1896-910.) 

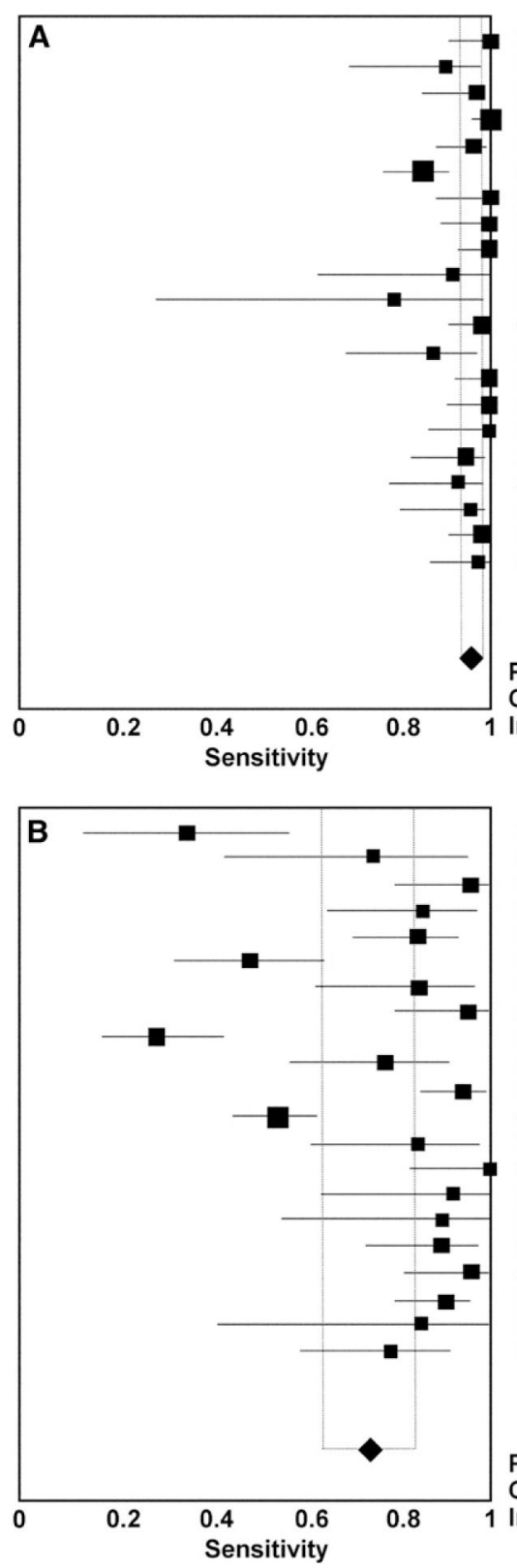

Nieman (2002 16-slice) Hoffman (2004 16-slice) Kuettner (2004 16-slice) Mollet (2004 16-slice) Hoffman (2005 16-slice) Kaiser (2005 16-slice) Mollet (2005 16-slice)

Morgan-H. (2005 16-slice)

Rodevand (2006 16-slice) Reant (2006 16-slice) Nikolaou (2006 16-slice) Garcia (2006 16-slice) Leber (2005 64-slice)

Leschka (2005 64-slice) Mollet (2005 64-slice)

Pugliese (2005 64-slice) Raff (2005 64-slice) Schuijf (2006 64-slice) Ropers (2006 64-slice) Ehara (2006 64-slice)

Nikolaou (2006 64-slice)
Sensitivity $(95 \% \mathrm{Cl})$

$1.00 \quad(0.91-1.00)$

$0.90 \quad 0.70-0.99$ )

$0.97 \quad 0.85-1.00)$

$1.00 \quad(0.97-1.00)$

$(0.88-1.00)$

$(0.78-0.92)$

$(0.89-1.00)$

(0.89-1.00)

$(0.93-1.00)$

$(0.64-1.00)$

(0.28-0.99)

$(0.91-1.00)$

$(0.69-0.97)$

$(0.92-1.00)$

$(0.91-1.00)$

$(0.86-1.00)$

(0.83-0.99)

(0.79- 0.99)

$(0.80-1.00)$

$(0.91-1.00)$

$(0.87-1.00)$

Pooled Sensitivity $=0.96(0.94$ to 0.98$)$

Chi-square $=57.96 ; \mathrm{df}=20(\mathrm{p}=0.0000)$

Inconsistency $(\mathrm{I}-\mathrm{square})=65.5 \%$

Sensitivity $(95 \% \mathrm{Cl})$

Nieman (2002 16-slice) $\quad 0.35 \quad(0.15-0.59)$ Hoffman (2004 16-slice) $\quad 0.75 \quad(0.43-0.95)$ Kuettner (2004 16-slice) $\quad 0.96 \quad(0.79-1.00)$ Mollet (2004 16-slice) $\quad 0.86 \quad(0.64-0.97)$ Hoffman (2005 16-slice) $\quad 0.84 \quad(0.71-0.94)$ Kaiser (2005 16-slice) $\quad 0.49 \quad(0.32-0.66)$ Mollet (2005 16-slice) $\quad 0.85 \quad(0.62-0.97)$ Morgan-H. (2005 16-slice) $\quad 0.96 \quad(0.80-1.00)$ Rodevand (2006 16-slice) $\quad 0.29 \quad(0.17-0.43)$ $\begin{array}{lll}\text { Reant (2006 16-slice) } & 0.78 \quad(0.58-0.91)\end{array}$ Nikolaou (2006 16-slice) $\quad 0.95 \quad(0.85-0.99)$ Garcia (2006 16-slice) $\quad 0.55 \quad(0.46-0.64)$ Leber (2005 64-slice) $\quad 0.85 \quad(0.62-0.97)$ Leschka (2005 64-slice) $\quad 1.00 \quad(0.83-1.00)$ $\begin{array}{lll}\text { Mollet (2005 64-slice) } & 0.92 \quad(0.64-1.00)\end{array}$ Pugliese (2005 64-slice) $\quad 0.90 \quad(0.55-1.00)$ Raff (2005 64-slice) $\quad 0.90 \quad(0.73-0.98)$ Schuijf (2006 64-slice) $\quad 0.97 \quad(0.82-1.00)$ Ropers (2006 64-slice) $\quad 0.91 \quad(0.80-0.97)$ Ehara (2006 64-slice) $\quad 0.86 \quad(0.42-1.00)$ Nikolaou (2006 64-slice) $\quad 0.79 \quad(0.60-0.92)$

Pooled Specificity $=0.74(0.65$ to 0.84$)$ Chi-square $=176.39 ; d f=20(p=0.0000)$ Inconsistency $(\mathrm{I}$-square $)=\mathbf{8 8 . 7 \%}$

FIG 17.

Plot and table of per-patient sensitivity (A) and specificity (B) of CCTA compared with invasive selective coronary angiography. Abbreviations as in Fig 16. (Reprinted with permission from Hamon M, Biondi-Zoccai GG, Malagutti P, et al. Diagnostic performance of multislice spiral computed tomography of coronary arteries as compared with conventional invasive coronary angiography: a metal-analysis. J Am Coll Cardiol 2006;48:1896-910.) 

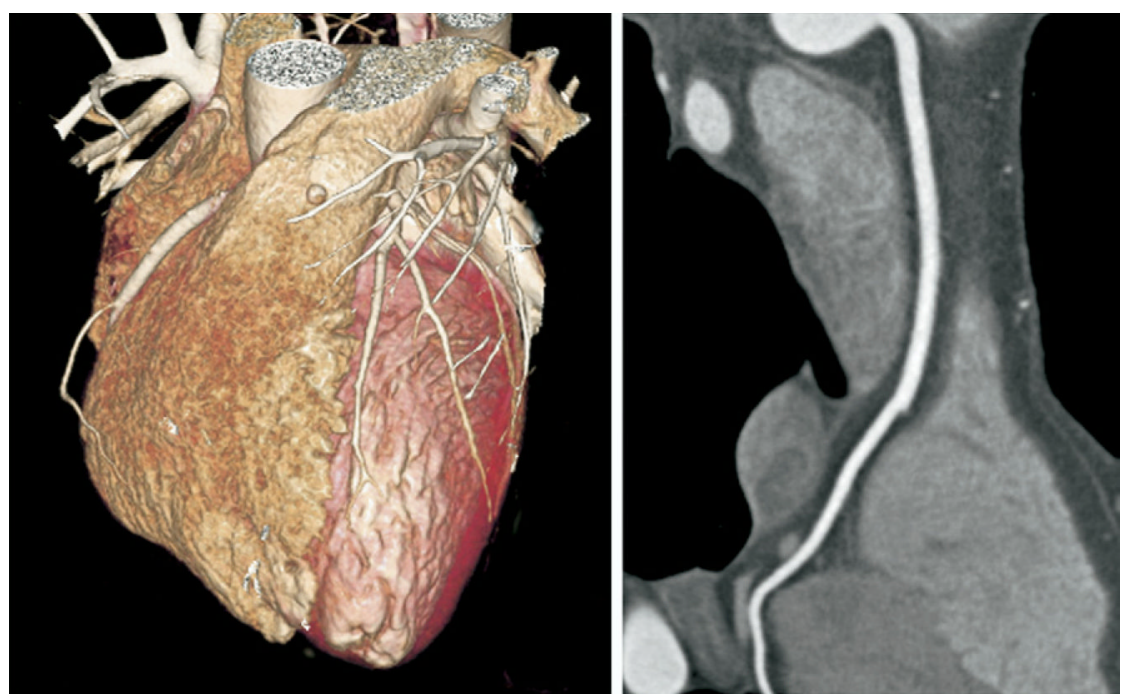

FIG 18.

Clinical experience confirms the high negative-predictive value of coronary $\mathrm{CT}$ angiography (CCTA), as shown in this figure; a high-quality normal CCTA can exclude plaque accumulation in the vessel wall and luminal stenosis. 

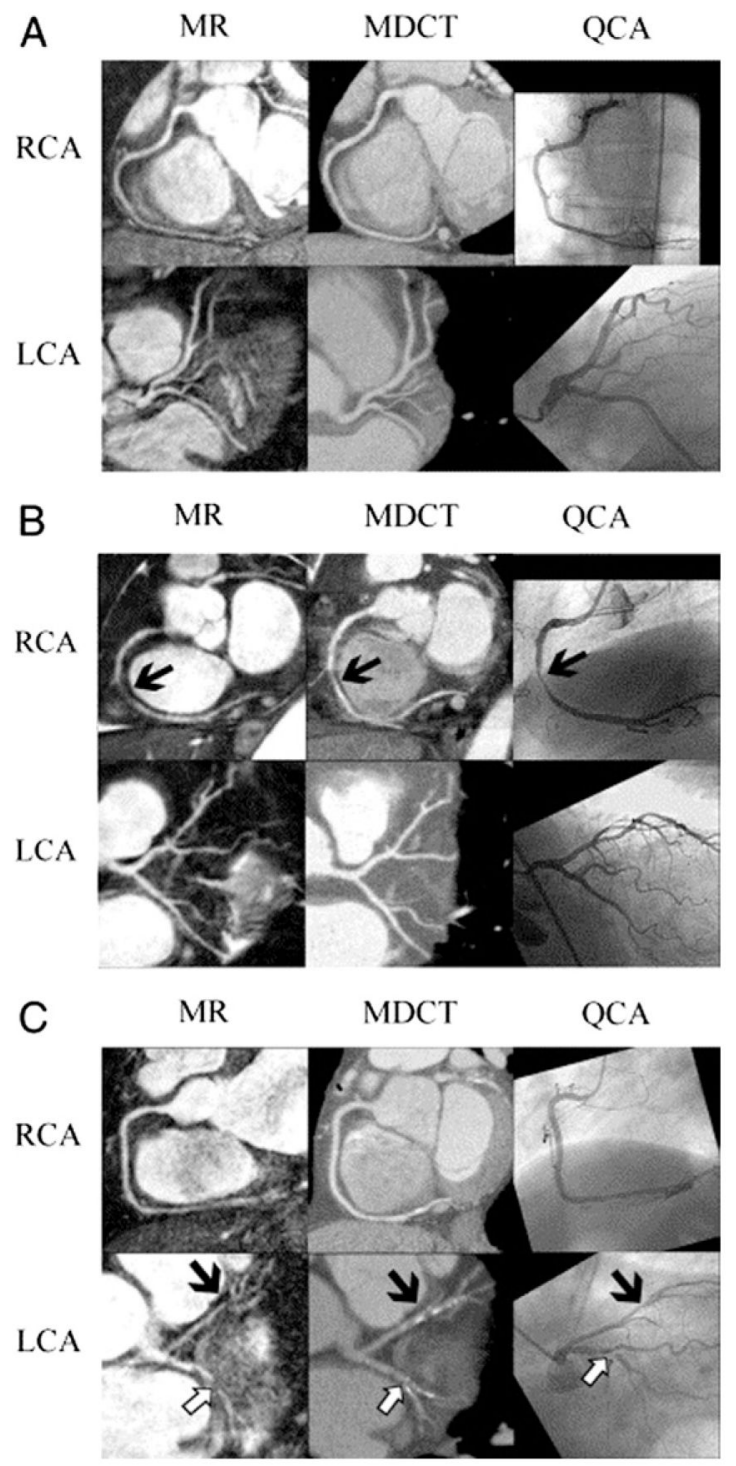

\section{FIG 19.}

Typical examples of reformatted coronary magnetic resonance (left panels), and multidetector CT (MDCT) (center panels) coronary angiography and corresponding quantitative coronary angiography images (right panels). The right (RCA, top panels) and left coronary artery systems (LCA, bottom panels) are shown. (A) Normal right and left coronary arteries by MR, MDCT, and quantitative coronary angiography. (B) Isolated mid-RCA stenosis (arrows). (C) Two-vessel disease involving the mid-LAD (black arrows), and left circumflex coronary artery (white arrows). (Reprinted with permission from Kefer J, Coche E, Legros G, et al. Head-tohead comparison of three-dimensional navigator-gated magnetic resonance imaging and 16slice computed tomography to detect coronary artery stenosis in patients. J Am Coll Cardiol 2005;46:92-100.) 

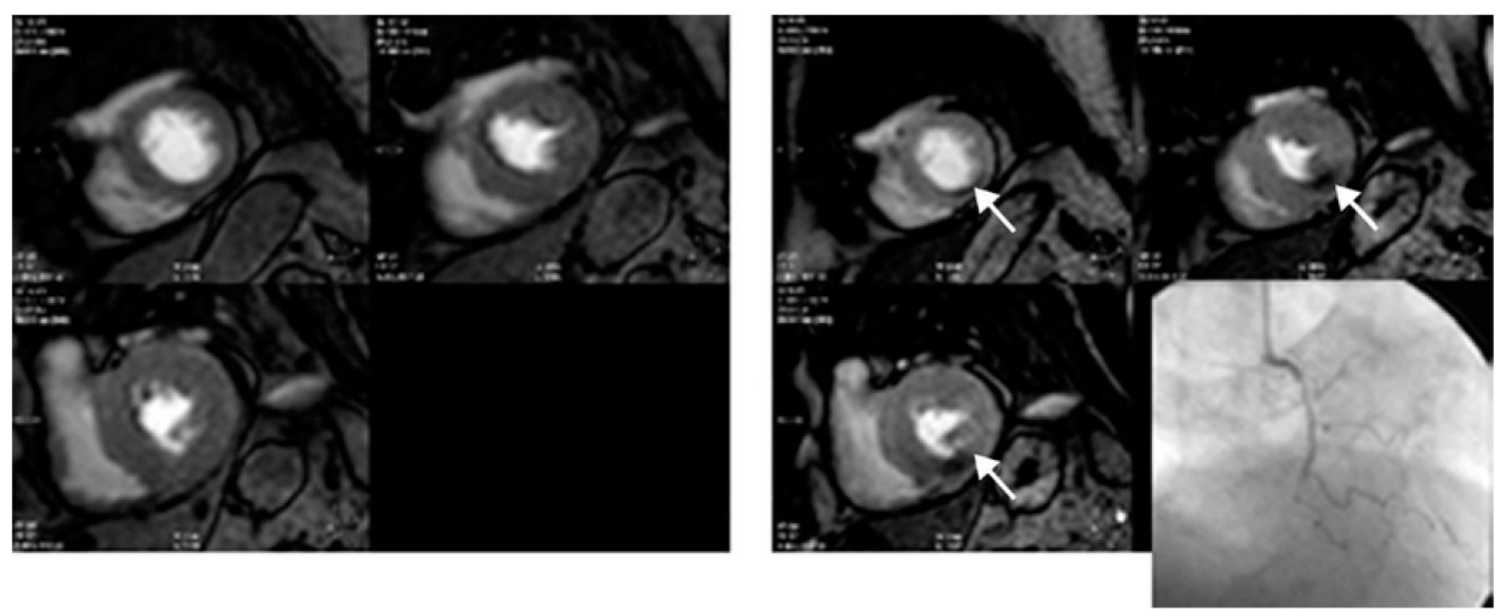

FIG 20.

First-pass myocardial perfusion at rest (left panel) and after adenosine stress (right panel). A reversible inferior perfusion defect (arrows) extends from apex to base. Right lower panel, the occluded right coronary artery of this symptomatic patient, imaged by selective angiography in right anterior oblique projection is shown. 
ED

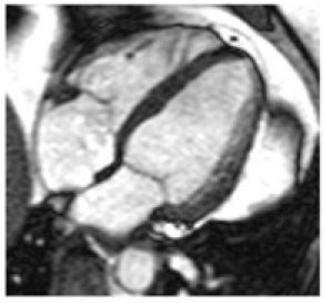

rest

$20 \mu \mathrm{g}$
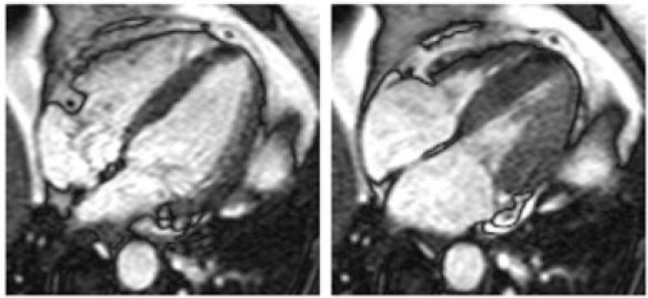

$40 \mu \mathrm{g}$
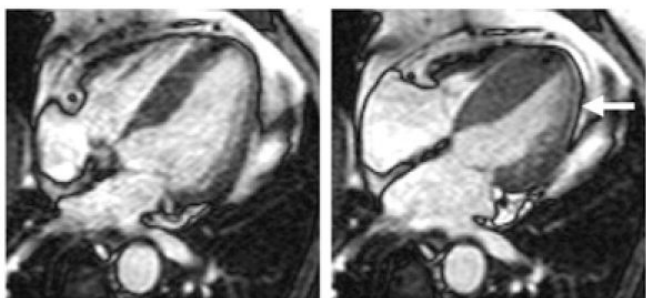

midventricular short axis

ED
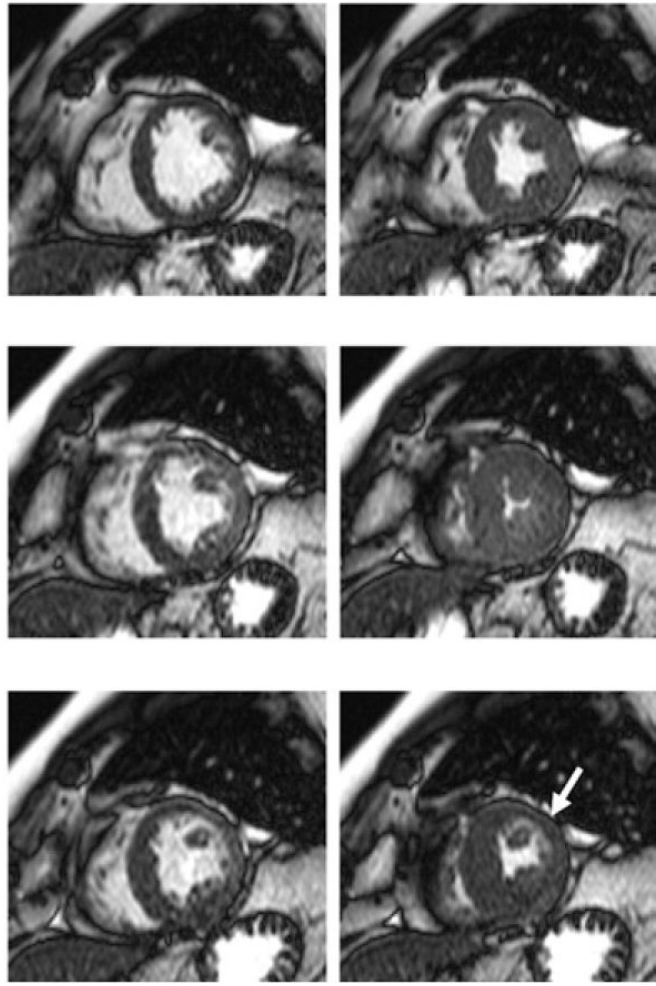

FIG 21.

Four-chamber and midventricular short-axis views at rest, and at intermediate- and peak-dose dobutamine stress (steady-state free precession technique). Both end-diastolic (ED) and endsystolic (ES) frames are shown. Note the development of midlateral regional wall motion abnormalities (arrows) at peak dobutamine stress. In this patient, invasive coronary angiography demonstrated a left circumflex coronary artery stenosis. (Reprinted with permission from Wahl A, Paetsch I, Gollesch A, et al. Safety and feasibility of high-dose dobutamine-atropine stress cardiovascular magnetic resonance for diagnosis of myocardial ischaemia: experience in 1000 consecutive cases. Eur Heart J 2004;25:1230-6.) 


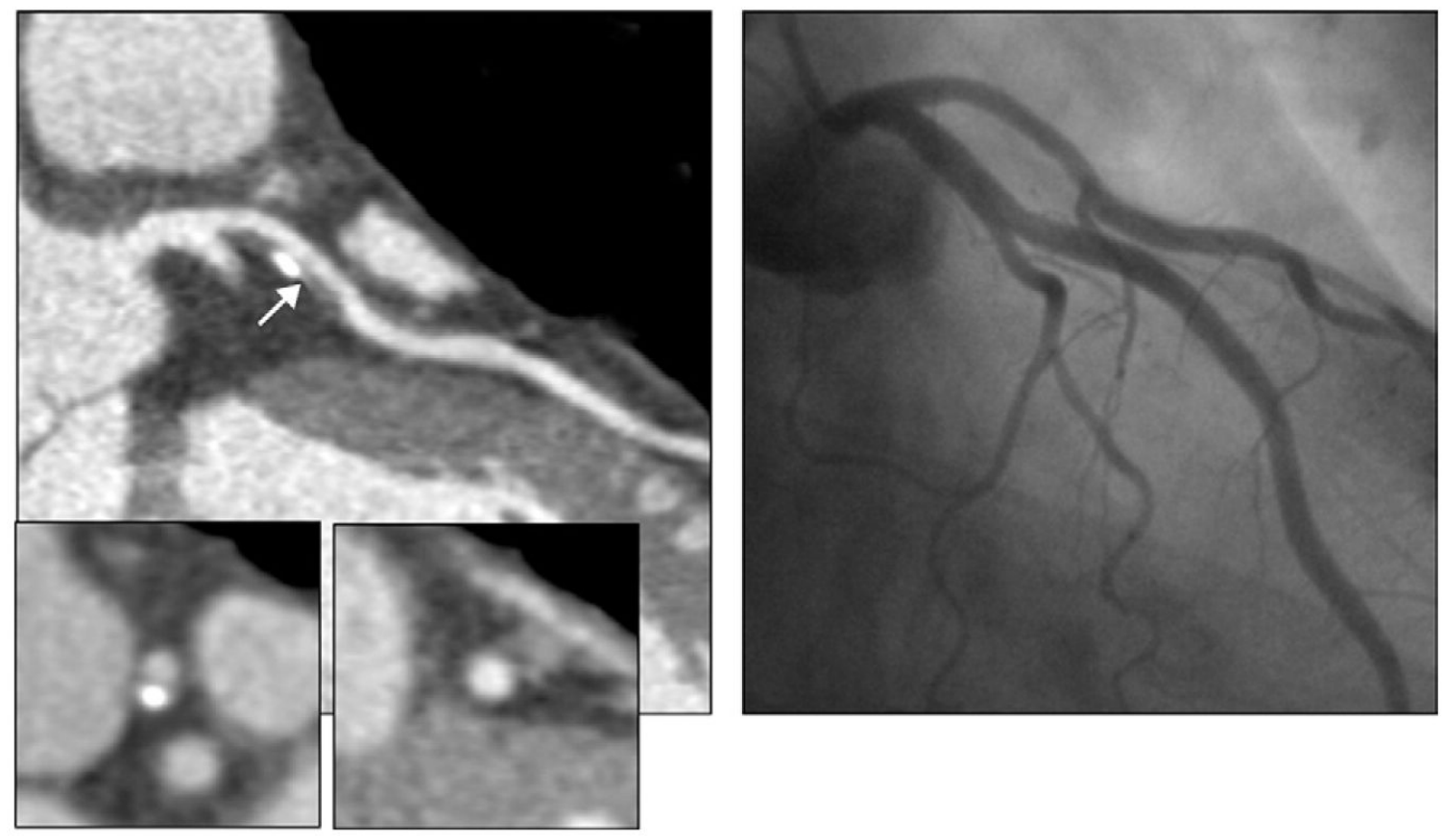

FIG 22.

Large, partially calcified plaque (arrow) of the proximal left anterior descending artery. The prognostic value of this finding and its implications for the management of patients with partially obstructive noncalcified plaque are unclear. 

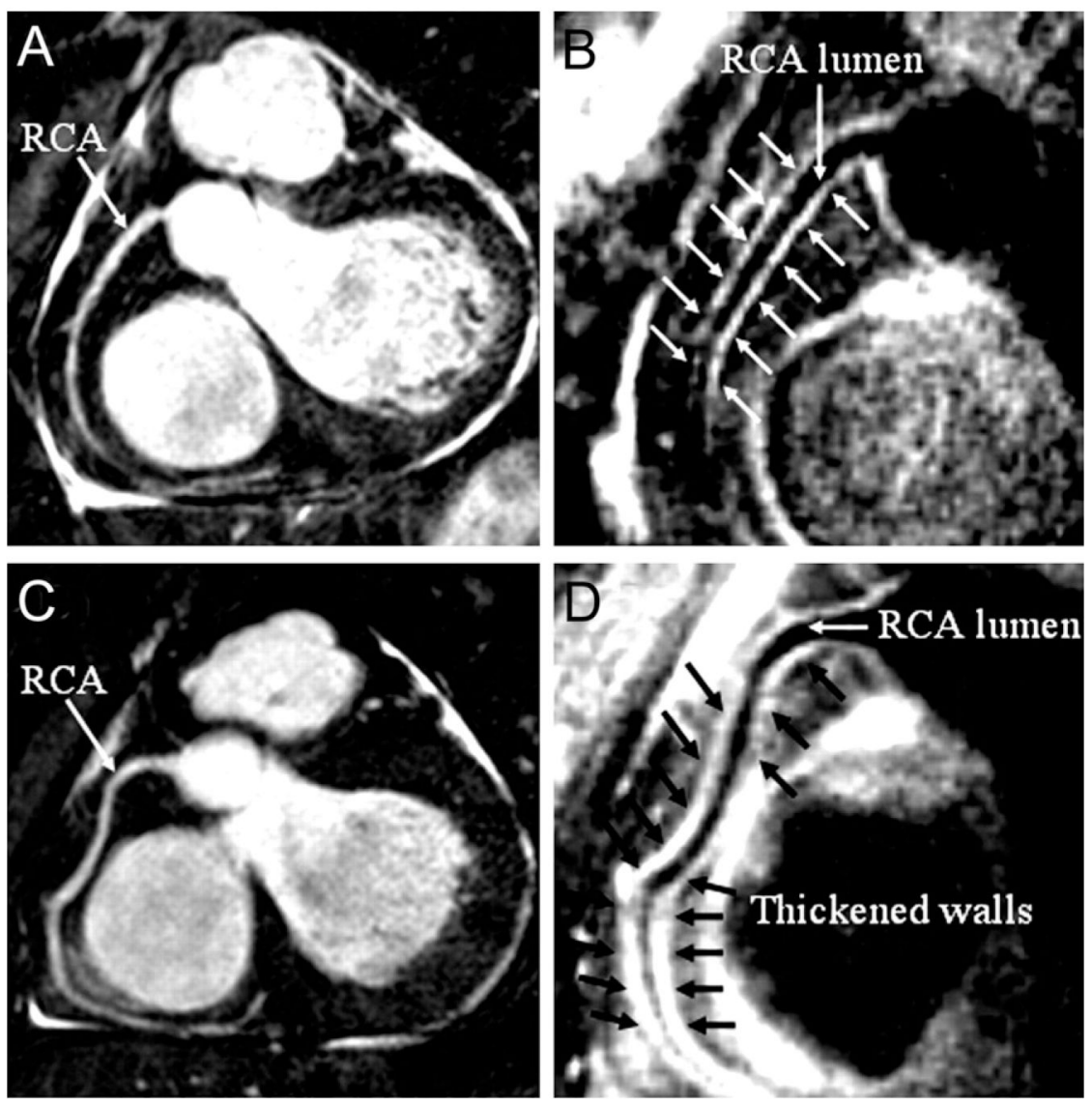

\section{FIG 23.}

Three-dimensional reformatted coronary MRI of the proximal right coronary artery (RCA) in two subjects without coronary luminal stenosis: a 58-year-old man with longstanding Type 1 diabetes and normoalbuminuria (A) and a 44-year-old man with longstanding Type 1 diabetes and diabetic nephropathy $(\mathrm{C})$. The corresponding three-dimensional black-blood vessel wall scans show no cardiac MRI evidence of atherosclerotic plaque (B; average and maximum vessel wall thickness, 1.1 and $1.3 \mathrm{~mm}$, respectively) and an increased atherosclerotic plaque burden ( $\mathrm{D}$; average and maximum vessel wall thickness, 2.3 and $3.0 \mathrm{~mm}$, respectively). The anterior and posterior RCA walls are indicated by arrows. (Reprinted with permission from Kim WY, Astrup AS, Stuber M, et al. Subclinical coronary and aortic atherosclerosis detected by magnetic resonance imaging in Type 1 diabetes with and without diabetic nephropathy. Circulation 2007;115:228-35.) 
TABLE 1

Radiation dose of cardiovascular radionuclide studies

\begin{tabular}{lc} 
Study & $\begin{array}{c}\text { Total body effective dose } \\
\text { (mSv) }\end{array}$ \\
\hline Tc-99m sestamibi 1-day rest-stress $(10+30 \mathrm{mCi})$ & 12 \\
Tc-99m sestamibi 2-day stress-rest $(30+30 \mathrm{mCi})$ & 17.5 \\
TL-201 stress and reinjection $(3.0+1.0 \mathrm{mCi})$ & $25.1^{a}$ \\
Dual-isotope (3.0 mCi TL-201 + 30 mCi Tc-99m) & 27.3 \\
Rb-82 PET myocardial perfusion (45 + 45 mCi) & $16^{b}$ \\
CT transmission source for PET (low-dose CT protocol) & 0.8 \\
Fluorine-18 fluorodeoxyglucose PET viability (10 mCi) & 7 \\
Radionuclide angiogram, Tc-99m-labeled red blood cells (20 mCi Tc-99m) & 5.2 \\
Ventilation/perfusion lung (200 mBq Tc-99m MAA + 70 MBq Tc-99m aerosol) & 2.8 \\
\hline
\end{tabular}

MAA, macroaggregated albumin.

(Reprinted with permission from Thompson RC, Cullom SJ. Issues regarding radiation dosage of cardiac nuclear and radiography procedures. J Nucl Cardiol 2006;13:19-23.)

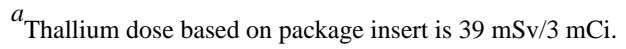

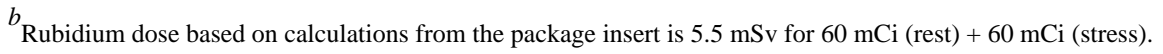


TABLE 2

Myocardial ischemia without epicardial coronary artery stenosis

\begin{tabular}{|c|c|}
\hline Disease & Mechanism for ischemia \\
\hline \multirow[t]{15}{*}{ Syndrome X } & Microvascular dysfunction \\
\hline & Microvascular spasm \\
\hline & Endothelial dysfunction \\
\hline & Estrogen deficiency \\
\hline & Increased sympathetic tone \\
\hline & Diffuse epicardial and microvascular coronary constriction \\
\hline & Structural abnormalities in coronary microvessels \\
\hline & Inadequate vasodilatory capacity \\
\hline & Nonischemic mechanisms \\
\hline & Abnormal interstitial potassium release \\
\hline & Adenosine release \\
\hline & Early cardiomyopathy \\
\hline & Increased pain perception \\
\hline & Myocardial metabolic abnormality \\
\hline & Insulin resistance \\
\hline \multirow[t]{2}{*}{ Diabetes mellitus } & Alterations of microvessels \\
\hline & Impaired microcirculatory coronary vasodilation \\
\hline \multirow[t]{2}{*}{ Left ventricular hypertrophy } & Increased demand \\
\hline & Inadequate vasodilatory capacity \\
\hline \multirow[t]{5}{*}{ Systemic lupus erythematosus } & Thrombi (hypercoagulation, antiphospholipid antibodies) \\
\hline & Vasospasm \\
\hline & Arteritis \\
\hline & Hypoestrogenism \\
\hline & Reduced endothelial function \\
\hline
\end{tabular}


TABLE 3

Diagnostic accuracy of adenosine stress first pass myocardial perfusion imaging with CMR

\begin{tabular}{lccll}
\hline Author & n & Sensitivity & Specificity & Comments \\
\hline Al-Saadi $^{149}$ & 40 & 92 & 87 & Selected patients \\
Schwitter $^{152}$ & 66 & 91 & 94 & Comparison with PET \\
Ibrahim $^{150}$ & $10+25$ & 86 & 84 & Healthy volunteers versus known CAD \\
Nagel $^{69}$ & 84 & 88 & 90 & ROC analysis, selected patients \\
Wolff $^{63}$ & 33 & 93 & 75 & ROC analysis, dose finding multicenter \\
Giang $^{147}$ & 51 & 93 & 75 & ROC analysis, dose finding multicenter \\
Schwitter $^{a}$ & 44 & 91 & 67 & ROC analysis, dose finding multicenter \\
Paetsch $^{70}$ & 79 & 91 & 62 & ROC analysis \\
Plein $^{151}$ & 92 & 88 & 82 & Including delayed enhancement \\
Klem $^{66}$ & 92 & 89 & 87 & \\
\end{tabular}

$R O C$, receiver operator curve.

${ }^{a}$ Presented at the European Heart Congress 2004. 
TABLE 4

Diagnostic accuracy of dobutamine stress magnetic resonance imaging

\begin{tabular}{lcccl}
\hline Author & n & Sensitivity & Specificity & Comments \\
\hline van Rugge $^{167}$ & 39 & 91 & 80 & \\
Nagel $^{162}$ & 208 & 86 & 86 & $\begin{array}{l}\text { Significantly superior to dobutamine } \\
\text { stress echocardiography }\end{array}$ \\
Hundley $^{164}$ & 153 & 83 & 83 & Patients unsuited for echocardiography \\
Paetsch $^{70}$ & 79 & 89 & 80 & $\begin{array}{l}\text { Favorable comparison to perfusion } \\
\text { imaging }\end{array}$ \\
Wahl $^{163}$ & 160 & 89 & 84 & Patients with wall motion abnormalities \\
Paetsch $^{165}$ & 150 & 78 & 87 & Multicenter interpretation \\
\hline
\end{tabular}


TABLE 5

Interpretation and recommendations for coronary artery calcium scoring

1 A negative test $($ score $=0$ ) makes the presence of atherosclerotic plaque, including unstable or vulnerable plaque, highly unlikely

2 A negative test (score $=0$ ) makes the presence of significant luminal obstructive disease highly unlikely (negative predictive power by EBCT approximately $95-99 \%$ ).

3 A negative test is consistent with a low risk ( $0.1 \%$ per year) of a cardiovascular event in the next $2-5$ years.

4 A positive test $(\mathrm{CAC}>0)$ confirms the presence of a coronary atherosclerotic plaque.

5 The greater the amount of coronary calcium, the greater the atherosclerotic burden in men and women, irrespective of age.

6 The total amount of coronary calcium correlates best with the total amount of atherosclerotic plaque, although the true "atherosclerotic burden" is underestimated.

7 A high calcium score (an Agatston score $>100$ ) is consistent with a high risk of a cardiac event within the next 2-5 years $(>2 \%$ annual risk).

8 CAC measurement can improve risk prediction in conventional intermediate-risk patients, and CAC scanning should be considered in individuals at intermediate risk for a coronary event (1.0\% per year to $2.0 \%$ per year) for clinical decision-making with regard to refinement of risk assessment.

9 Decisions for further testing (such as stress testing or cardiac catheterization) beyond assistance in risk stratification in patients with a positive CAC score cannot be made by coronary calcium scores alone, as calcium score correlates poorly with stenosis severity in a given individual and should be based on clinical history and other conventional clinical criteria.

$C A C$, coronary artery calcification; $E B C T$, electron beam computed tomography.

(Reprinted with permission from Budoff MJ, Achenbach S, Blumenthal RS, et al. Assessment of coronary artery disease by cardiac computed tomography: a scientific statement from the American Heart Association Committee on Cardiovascular Imaging and Intervention, Council on Cardiovascular Radiology and Intervention, and Committee on Cardiac Imaging, Council on Clinical Cardiology. Circulation 2006;114:1761-91.) 
TABLE 6

Indications for cardiac magnetic resonance

Congenital heart disease

General

Initial evaluation and follow-up of adult congenital heart disease

Specific

1 Assessment of shunt size (Qp/qs)

2 Anomalies of the viscero-atrial situs:

Situs anomalies with complex congenital heart disease

3 Anomalies of the atria and venous return

Anomalous pulmonary venous return, especially in complex anomalies and cor triatriatum

Anomalous systemic venous return

Systemic or pulmonary venous obstruction following intra-atrial baffle repair or correction of anomalous pulmonary venous return

4 Anomalies of the ventricles

VSD associated with complex anomalies

Supracristal VSD

Evaluation of right and left ventricular volumes, mass, and function

5 Anomalies of the semilunar valves

Pulmonary regurgitation

Supravalvular aortic stenosis

Coronary artery disease

1 Assessment of global ventricular (left and right) function and mass

2 Detection of coronary artery disease

Coronary MRA (anomalies)

3 Acute and chronic myocardial infarction

Detection and assessment

Myocardial viability

Pericardial disease, cardiac tumors, cardiomyopathies, and cardiac transplants

1 Detection and characterization of cardiac and pericardiac tumors

2 Hypertrophic cardiomyopathy

Apical

3 Dilated cardiomyopathy

Differentiation from dysfunction related to coronary artery disease

4 Arrhythmogenic right ventricular cardiomyopathy (dysplasia)

5 Siderotic cardiomyopathy (in particular thalassemia)

Valvular heart disease

Curr Probl Cardiol. Author manuscript; available in PMC 2009 July 13. 
1 Cardiac chamber anatomy and function

2 Quantification of regurgitation

$M R A$, magnetic resonance angiography; $Q p / q s$, ratio of pulmonary to systemic flow; $V S D$, ventricular septal defect.

(Reprinted with permission from Pennell DJ, Sechtem UP, Higgins CB, et al. Clinical indications for cardiovascular magnetic resonance (CMR): Consensus Panel report. Eur Heart J 2004;25:1940-65.) 
TABLE 7

Appropriate indications for coronary and chest CTA and cardiac CT

Type of examination

Score $^{a}$

Coronary CTA

Evaluation of suspected coronary anomalies

Chest pain syndrome in patients with uninterpretable or equivocal stress test (exercise, perfusion, or stress echo)

Chest pain syndrome in patients with intermediate pretest probability of CAD where ECG uninterpretable OR who are unable to exercise

Acute chest pain in patients with intermediate pretest probability of CAD but no ECG changes and negative serial enzymes

Assessment of complex congenital heart disease including anomalies of coronary circulation, great vessels, and cardiac

chambers and valves

Evaluation of coronary arteries in patients with new onset heart failure to assess etiology

Vascular CTA

Evaluation of suspected aortic dissection or thoracic aortic aneurysm

Evaluation of suspected pulmonary embolism

Cardiac $C T$

Evaluation of cardiac mass (suspected tumor or thrombus) in patients with technically limited images from echocardiogram,

Evaluation of pericardial conditions (pericardial mass, constrictive pericarditis, or complications of cardiac surgery) in patients with technically limited images from echocardiogram, MRI, or TEE

Evaluation of pulmonary vein anatomy before invasive radiofrequency ablation for atrialfibrillation

Noninvasive coronary vein mapping before placement of biventricular pacemaker

Noninvasive coronary arterial mapping, including internal mammary artery before repeat cardiac surgical revascularization

$C A D$, coronary artery disease; $C T$, computed tomography; $C T A, \mathrm{CT}$ angiography; $E C G$, electrocardiogram; $M R I$, magnetic resonance imaging; $T E E$, transesophageal echocardiogram.

(Modified with permission from Hendel RC, Patel MR, Kramer CM, et al. ACCF/ACR/SCCT/SCMR/ASNC/NASCI/SCAI/SIR 2006 appropriateness criteria for cardiac computed tomography and cardiac magnetic resonance imaging: a report of the American College of Cardiology Foundation Quality Strategic Directions Committee Appropriateness Criteria Working Group, American College of Radiology, Society of Cardiovascular Computed Tomography, Society for Cardiovascular Magnetic Resonance, American Society of Nuclear Cardiology, North American Society for Cardiac Imaging, Society for Cardiovascular Angiography and Interventions, and Society of Interventional Radiology. J Am Coll Cardiol 2006;48:1475-97.)

${ }^{a}$ Median score assigned by panel members. 
TABLE 8

Appropriate indications for cardiac and coronary MRI

Type of examination

Score $^{a}$

Vasodilator Perfusion CMR or Dobutamine Stress CMR

Chest pain syndrome in patients with intermediate pretest probability of CAD where ECG is uninterpretable OR who are unable to exercise

Patients who had coronary angiography (catheterization or CT) showing a stenosis of unclear significance

Coronary MR Angiography

Evaluation of suspected coronary anomalies
Delayed Myocardial Enhancement MRI

To determine viability before revascularization to establish likelihood of recovery of function with revascularization (PCI or $\mathrm{CABG}$ ) or medical therapy

To determine viability before revascularization when viability assessment by SPECT or dobutamine echo has provided "equivocal or indeterminate" results

To determine the location and extent of myocardial necrosis including "no reflow" regions in patients after acute myocardial infarction

Combinations of CMR Techniques

Assessment of complex congenital heart disease including anomalies of coronary circulation, great vessels, and cardiac chambers and valves

Evaluation for arrythmogenic right ventricular cardiomyopathy in patients presenting with syncope or ventricular arrhythmia

Evaluation of cardiac mass (suspected tumor or thrombus)

Evaluation of LV function following myocardial infarction OR in heart failure patients with technically limited images from echocardiogram

Quantification of LV function in patients with discordant information that is clinically significant from prior tests

Evaluation of specific cardiomyopathies (infiltrative (amyloid, sarcoid), HCM, or due to cardiotoxic therapies)

Characterization of native and prosthetic cardiac valves - including planimetry of stenotic disease and quantification of regurgitant disease in patients with technically limited images from echocardiogram or TEE

Evaluation of myocarditis or myocardial infarction with normal coronary arteries in patients with positive cardiac enzymes who have no obstructive atherosclerosis on angiography

Evaluation of pericardial conditions (pericardial mass, constrictive pericarditis)

Evaluation for aortic dissection

Evaluation of pulmonary veins before radiofrequency ablation for atrial fibrillation to assess left atrial and pulmonary venous anatomy including dimensions of veins for mapping purposes

$C A B G$, coronary artery bypass grafting; $C M R$, cardiac magnetic resonance; $C T$, computed tomography; $E C G$, electrocardiogram; $H C M$, hypertrophic cardiomyopathy; $L V$, left ventricle; $M R I$, magnetic resonance imaging; $P C I$, percutaneous coronary intervention; $S P E C T$, single-photon emission computed tomography; TEE, transesophageal echocardiogram.

(Modified with permission from Hendel RC, Patel MR, Kramer CM, et al. ACCF/ACR/SCCT/SCMR/ASNC/NASCI/SCAI/SIR 2006 appropriateness criteria for cardiac computed tomography and cardiac magnetic resonance imaging: a report of the American College of Cardiology Foundation Quality Strategic Directions Committee Appropriateness Criteria Working Group, American College of Radiology, Society of Cardiovascular Computed

Tomography, Society for Cardiovascular Magnetic Resonance, American Society of Nuclear Cardiology, North American Society for Cardiac Imaging, Society for Cardiovascular Angiography and Interventions, and Society of Interventional Radiology. J Am Coll Cardiol 2006;48:1475-97.

${ }^{a}$ Median score assigned by panel members.

Curr Probl Cardiol. Author manuscript; available in PMC 2009 July 13. 